\title{
Symmetries and charges of general relativity at null boundaries
}

\author{
Venkatesa Chandrasekaran, ${ }^{a}$ Éanna É. Flanagan ${ }^{b, c}$ and Kartik Prabhu ${ }^{c}$ \\ ${ }^{a}$ Center for Theoretical Physics and Department of Physics, University of California, \\ Berkeley, CA 94720, U.S.A. \\ ${ }^{b}$ Department of Physics, Cornell University, \\ Ithaca, NY 14853, U.S.A. \\ ${ }^{c}$ Cornell Laboratory for Accelerator-based Sciences and Education (CLASSE), Cornell University, \\ Ithaca, NY 14853, U.S.A. \\ E-mail: ven_chandrasekaran@berkeley.edu, eef3@cornell.edu, \\ kartikprabhu@cornell.edu
}

ABSTRACT: We study general relativity at a null boundary using the covariant phase space formalism. We define a covariant phase space and compute the algebra of symmetries at the null boundary by considering the boundary-preserving diffeomorphisms that preserve this phase space. This algebra is the semi-direct sum of diffeomorphisms on the two sphere and a nonabelian algebra of supertranslations that has some similarities to supertranslations at null infinity. By using the general prescription developed by Wald and Zoupas, we derive the localized charges of this algebra at cross sections of the null surface as well as the associated fluxes. Our analysis is covariant and applies to general non-stationary null surfaces. We also derive the global charges that generate the symmetries for event horizons, and show that these obey the same algebra as the linearized diffeomorphisms, without any central extension. Our results show that supertranslations play an important role not just at null infinity but at all null boundaries, including non-stationary event horizons. They should facilitate further investigations of whether horizon symmetries and conservation laws in black hole spacetimes play a role in the information loss problem, as suggested by Hawking, Perry, and Strominger.

KeYwords: Black Holes, Classical Theories of Gravity, Space-Time Symmetries

ARXIV EPRINT: 1807.11499 


\section{Contents}

1 Introduction 1

1.1 Notation and conventions 4

2 Review of the covariant phase space formalism 4

2.1 Definitions of field configuration space and covariant phase space 4

$\begin{array}{ll}2.2 & \text { Definitions of currents }\end{array}$

$\begin{array}{ll}2.3 & \text { Definition of presymplectic form on covariant phase space }\end{array}$

2.4 Global charges that generate boundary symmetries 8

$\begin{array}{ll}2.5 & \text { Boundary symmetry algebras of linearized diffeomorphisms } \\ & 11\end{array}$

2.6 Localized (Wald-Zoupas) charges, fluxes and conservation laws 11

$\begin{array}{lll}2.7 & \text { Potential ambiguities in global and localized charges } & 15\end{array}$

3 Review of the local geometry of null hypersurfaces $\quad 16$

$\begin{array}{llr}3.1 \text { Foundations } & 16\end{array}$

$\begin{array}{lll}3.2 & \text { Geometric fields defined on a null hypersurface } & 17\end{array}$

$\begin{array}{lll}3.3 & \text { Divergence operator } & 19\end{array}$

3.4 Stationary regions of null hypersurfaces 20

$\begin{array}{ll}3.5 & \text { Orthonormal basis formalism }\end{array}$

4 Universal intrinsic structure of a null hypersurface $\quad 21$

$\begin{array}{ll}4.1 \text { Definition of intrinsic structure } & 21\end{array}$

4.2 Symmetry group of a complete intrinsic structure 23

4.3 Symmetry algebra of a complete intrinsic structure 24

4.4 Preferred subalgebra for stationary regions of a null hypersurface: Killing $\begin{array}{ll}\text { supertranslations } & 28\end{array}$

4.5 Symmetry groups of null hypersurfaces with boundaries 28

5 General relativity with a null boundary: covariant phase space $\quad 29$

$\begin{array}{lll}5.1 & \text { Definition of field configuration space } & 30\end{array}$

$\begin{array}{lll}5.2 & \text { Symmetry algebra of the field configuration space } & 32\end{array}$

5.3 Boundary conditions on the variation of the metric 34

6 Global and localized charges for a null boundary component 36

$\begin{array}{lll}6.1 & \text { Noether charge } & 36\end{array}$

$\begin{array}{ll}6.2 \text { Variation of Noether charge } & 37\end{array}$

6.3 Global charges that generate boundary symmetries 38

$\begin{array}{lll}6.4 & \text { Localized (Wald-Zoupas) charges and fluxes } & 39\end{array}$

6.5 Charges and fluxes for specific symmetry generators 41

6.6 Stationary regions of the null surface 42

7 Global conservation laws involving black holes $\quad 42$ 
8 Algebra of symmetry generator charges and central charges

8.1 Algebra of symmetry generator charges in general contexts 45

$\begin{array}{lll}\text { 8.2 Symmetry algebra of global charges at event horizons } & 47\end{array}$

8.3 Symmetry algebras of localized charges 48

9 Discussion, applications and future directions $\quad 48$

$\begin{array}{lll}9.1 & \text { Recap } & 48\end{array}$

9.2 Black holes: localized conservation laws and horizon memory 49

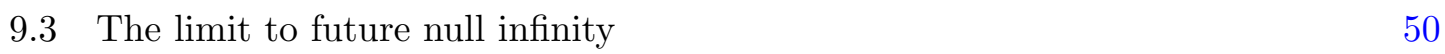

$\begin{array}{lll}9.4 & \text { Generalizations } & 51\end{array}$

A Orthonormal basis formalism for null surfaces $\quad \mathbf{5 2}$

A.1 Review of structures associated with a choice of auxiliary null vector $\quad 52$

A.2 Geometric fields on an orthonormal basis 53

A.3 Expressions for charges 53

B Gauge fixing in the definition of field configuration space $\quad 54$

C Characterization of trivial diffeomorphisms at a null boundary $\quad 55$

D Consistency check of symmetry algebra $\quad 56$

$\begin{array}{ll}\text { E Choice of reference solution } & 56\end{array}$

F Consistency of two expressions for flux of localized charge 58

G Symplectic currents on black holes horizons $\quad 59$

H Alternative definition of field configuration space and associated $\begin{array}{ll}\text { symmetry algebra } & 60\end{array}$

\section{Introduction}

It is well known that gauge transformations of a diffeomorphism invariant theory can become genuine symmetries of the theory at boundaries of the spacetime. In general relativity, diffeomorphisms of asymptotically flat spacetimes that preserve the fall-off conditions for the metric near null infinity yield the standard BMS group [1-3]. Similarly, in QED there exists an infinite set of symmetries at null infinity comprised of large gauge transformations $[4,5]$. Associated to the various symmetries are global conserved charges which act as generators of the symmetries $[6,7]$. There are in addition localized charges such as Bondi mass which quantify the amount of charge in subregions of the spacetime boundary, which can be calculated using a variety of formalisms $[6,8,9]$. 
More recently, it has been found that stationary black holes also possess an infinite number of symmetries beyond the usual horizon Killing symmetries [10-20] (see [21] for older work on this topic, and [22] for the electromagnetic case). The new symmetries are diffeomorphisms which preserve the near horizon geometry under specific gauge conditions, and a subclass of them are similar to the supertranslations at null infinity. These horizon supertranslations give rise to contributions to the global charges associated with supertranslations, in addition to the contribution from null infinity. In [14-16] it was suggested that this enlarged group of horizon symmetries and its associated charges and conservation laws play a role in how information is released as a black hole evaporates, and may lead to a resolution of the information loss paradox (see also [23, 24]). At the least, a complete analysis of supertranslation conservation laws in black hole spacetimes cannot be undertaken without first knowing what the supertranslation charges and fluxes are on general, non-stationary event horizons. It is therefore of considerable interest to gain a deeper, more unified understanding of such symmetries and charges.

A natural question is whether supertranslations are symmetries of general relativity at any null surface, with stationary horizons and null infinity being special cases. This would give null boundaries in general relativity quite a rich structure from the phase space point of view, and put supertranslations on far more general footing. As one of the main results of this paper, we systematically calculate the group and algebra of symmetries of general relativity at a null boundary at a finite location in spacetime, and show that this is indeed the case. We do so using covariant phase space methods, which clarifies the geometric meaning of the symmetries. The symmetry group is the semidirect product of the group of diffeomorphisms of the base space (typically the two-sphere) with a nonabelian group of supertranslations, which contains angle-dependent displacements of affine parameter as well as angle-dependent rescalings of affine parameter. ${ }^{1}$ The results apply to nonstationary black hole horizons as well as cosmological horizons.

We next turn to the charges and conservation laws associated with these symmetries. We distinguish between global charges and associated global conservation laws - the independence of integrals over Cauchy surfaces $\Sigma$ of the choice of Cauchy surface - and localized charges and localized conservation laws, which involve integrals over hypersurfaces $\Sigma$ that are not Cauchy surfaces. For the global charges, we compute explicitly the contribution to the charges from integrals over event horizons. The complete charges and complete formulation of the conservation laws requires an understanding of how the symmetries of the event horizon mesh with asymptotic symmetries at null infinity. This has been worked out in some special cases $[15,16]$, but the general case is a subject for future investigations.

Localized charges, for example the Bondi mass at cross sections of future null infinity, are associated with localized conservation laws that express the difference between the charges at two successive cross sections with the integral of a flux over the intervening region of the boundary. These charges are not generators of symmetries on phase space.

\footnotetext{
${ }^{1}$ Our symmetry group does not coincide exactly with any of the several different groups in refs. [10$13,19,21]$, since we preserve a particular geometric structure on the null surface which defines our field configuration space, and other authors preserve other quantities such as the near horizon geometry.
} 
Wald and Zoupas [6] give a general prescription for computing such charges, by starting with the integral of a symplectic current that defines the variation of the global charge, and restricting the domain of integration to a hypersurface which is not a Cauchy surface, in order to attempt to obtain the charge contained within some of the degrees of freedom of the theory. This quantity is not in general a total variation and so cannot be integrated up in phase space to obtain the charge. Wald and Zoupas give a prescription for adding a correction term that overcomes this obstacle, thus allowing the definition of finite charges. Their prescription gives the conventional answers for localized charges and fluxes at null infinity [6].

In this paper we describe how to adapt the prescription to a finite null surface, and calculate the charges and fluxes of the symmetry algebra at the surface. In particular, we obtain simple expressions for the supertranslation charges and fluxes. The result applies to a very general class of null surfaces including, most importantly, non-stationary event horizons. The fluxes manifestly satisfy the property that they vanish on stationary solutions at the null surface, as one would desire if the charges are to be physically meaningful.

An interesting question is the physical interpretation of the localized charges at the null surface. At null infinity, such an interpretation of supertranslation charges is provided by the memory effect. The supertranslation that relates two different stationary regions (or vacua) can be measured as a gravitational wave memory [24, 25]. Outgoing radiation can be though of as causing a transition from one vacuum to another. A similar situation likely occurs at a black hole horizon, when accretion of radiation causes a transition from one state to a supertranslated state, with the supertranslation being measurable by nearhorizon observers as a memory effect. While some aspects of this memory have been uncovered [16] there are still open questions.

Aside from the above motivations, which are centered around black holes, an understanding of the gravitational symmetry algebra at a null surface is important in and of itself: null surfaces play a crucial role in information theoretic constraints and dynamics within field theory and semi-classical gravity [26-28], in holographic settings and action formulations [29-31], in derivations of the generalized second law [27], and even in quantum gravity [32, 33]. The covariant phase space formalism for spacetimes with boundary is also important in studying the contribution of edge modes to entanglement entropy in gauge theories and gravity [34,35]. As such, a complete description of the symmetries and charges of general non-stationary solutions at null surfaces could provide further insight into gravity, just as it did at null infinity.

Our work is complementary to the recent derivation of Hopfmuller and Friedel of boundary currents for arbitrary null surfaces and associated local conservation laws, for arbitrary vector fields tangent to the null surface [36]. Earlier treatments of the symplectic structure of general relativity on null surfaces and in $2+2$ formulations can be found in refs. [37-40].

The paper is organized as follows. Section 2 reviews the covariant phase space formulation of boundary symmetries and conserved charges of diffeomorphism covariant theories, and section 3 establishes our conventions for describing the local geometry of null surfaces. In section 4 we define a universal intrinsic structure for null hypersurfaces, and derive its in- 
variance group and algebra. Section 5 defines a covariant phase space for general relativity with a null boundary, and shows the associated symmetry algebra of linearized diffeomorphisms is the same as that of the universal intrinsic structure. The global and localized charges associated with these symmetries are discussed in section 6, and global conservation laws in section 7. Section 8 shows that for event horizons, the algebra of global charges under Dirac brackets coincides with the algebra of linearized diffeomorphisms under Lie brackets. Section 9 discusses other applications to black holes and concludes.

\subsection{Notation and conventions}

We use the sign convention $(-,+,+,+)$ throughout. We use the following conventions for tensor indices:

- Tensors on the spacetime $M$ will be denoted by lowercase Roman abstract indices $a$, $b, c$ etc. from the first half of the alphabet.

- Tensors on the null surface $\mathcal{N}$ will be denoted by lowercase Roman abstract indices $i, j, k$ etc. from the second half of the alphabet.

- Tensors built on the vector space of covectors $w_{i}$ orthogonal to the normal $\ell^{i}$ at a point on $\mathcal{N}$ will be denoted by uppercase Roman abstract indices $A, B, C$ etc.

Boldface quantities like $\boldsymbol{\omega}$ will denote differential forms. In section 2 we will work in $d$ spacetime dimensions, but in the remainder of the paper we will specialize to 4 spacetime dimensions.

\section{Review of the covariant phase space formalism}

In this section we review the generally covariant phase space framework for describing symmetries in a diffeomorphism covariant theory on a manifold $M$ with boundary $\partial M[6$, 41-46]. We mostly follow the notations and terminology of Wald and Zoupas [6], with one or two exceptions noted below. The framework is very general and can be applied to arbitrary theories and boundary conditions. It was applied to vacuum general relativity at null infinity in ref. [6], and will be applied to vacuum general relativity at finite null boundaries in later sections of this paper.

A summary of the properties of the various charges and conservation laws reviewed in this section is given in table 1 .

\subsection{Definitions of field configuration space and covariant phase space}

We consider a $d$-dimensional manifold $M$ with boundary $\partial M$, on which we want to define a theory of some dynamical fields $\phi$, tensors ${ }^{2}$ on $M$ (we suppress tensor indices on $\phi$ ). In the following sections of the paper we will specialize to vacuum general relativity for which $\phi=g_{a b}$. The boundary of $M$ can consist of a number of different components $\mathcal{B}_{j}$,

$$
\partial M=\cup_{j} \mathcal{B}_{j} .
$$

\footnotetext{
${ }^{2}$ One can also include dynamical fields that are gauge-covariant fields defined on a principal bundle over $M[47]$.
} 


\begin{tabular}{|c|c|c|c|c|}
\hline Property & Noether charge & $\begin{array}{l}\text { Boundary symmetry } \\
\text { "charge variation" }\end{array}$ & $\begin{array}{c}\text { Localized } \\
\text { (Wald-Zoupas) } \\
\text { boundary symmetry } \\
\text { charge }\end{array}$ & $\begin{array}{l}\text { Global symmetry } \\
\text { generator charge }\end{array}$ \\
\hline Symbol & $Q_{\xi}$ & $\not \mathcal{Q}_{\xi, j}{ }^{a}$ & $\mathcal{Q}_{\xi}^{\text {loc }}$ & $\mathcal{Q}_{\xi}$ \\
\hline $\begin{array}{c}\text { Defining } \\
\text { equations }\end{array}$ & $(2.6),(2.7),(6.7)$ & $(2.14)$ & $\begin{array}{c}(2.25),(2.24),(2.26), \\
(2.27)\end{array}$ & $\begin{array}{l}\text { (2.13) with } \Sigma \text { a } \\
\text { Cauchy surface }\end{array}$ \\
\hline $\begin{array}{l}\text { Always well } \\
\text { defined? }\end{array}$ & Yes & Yes & $\begin{array}{l}\text { Requires the existence } \\
\text { of presymplectic } \\
\text { potential } \Theta \text { satisfying } \\
\text { certain properties }\end{array}$ & $\begin{array}{c}\text { Yes (assuming validity } \\
\text { of conjecture of } \\
\text { section 2.6) }\end{array}$ \\
\hline $\begin{array}{l}\text { Interpretation as } \\
\text { generator of } \\
\text { symmetry? }\end{array}$ & No & No & No & Yes \\
\hline Depends on? & $\begin{array}{c}\text { Field configuration } \phi, \\
(d-2) \text {-surface } \mathcal{S} \\
\text { boundary symmetry } \\
\xi^{a} \text { at } \mathcal{S}\end{array}$ & $\begin{array}{l}\text { Field configuration } \phi, \\
\text { field variation } \delta \phi, \\
(d-2) \text {-surface } \mathcal{S} \\
\text { boundary symmetry } \\
\qquad \xi^{a} \text { at } \mathcal{S}\end{array}$ & $\begin{array}{c}\text { Field configuration } \phi, \\
(d-2) \text {-surface } \mathcal{S} \\
\text { boundary symmetry } \\
\xi^{a} \text { at } \mathcal{S}\end{array}$ & $\begin{array}{c}\text { Field configuration } \phi \\
\text { global boundary } \\
\text { symmetry } \xi^{a} \\
\text { (assuming global } \\
\text { conservation laws } \\
\text { valid) }\end{array}$ \\
\hline $\begin{array}{c}\text { Nature of } \\
\text { associated } \\
\text { conservation law }\end{array}$ & $\begin{array}{l}\text { Conserved Noether } \\
\text { current }(2.6) \text { on } \\
\text { spacetime }\end{array}$ & $\begin{array}{c}\text { Conserved } \\
\text { presymplectic } \\
\text { current }(2.8) \text { on } \\
\text { spacetime }\end{array}$ & $\begin{array}{c}\text { Exact }(d-1)- \\
\text { form }(2.25),(2.29) \text { on } \\
\text { component of } \\
\text { boundary }\end{array}$ & $\begin{array}{l}\text { Conjectured law is } \\
\text { that integral of } \\
\text { symplectic } \\
\text { current }(2.8) \text { over } \\
\text { Cauchy surface } \Sigma \text { and } \\
\text { then in phase space } \\
\text { independent of } \Sigma \\
\text { (section } 7 \text { ). } \\
\text { Established in some } \\
\text { special cases }\end{array}$ \\
\hline
\end{tabular}

${ }^{a}$ The slash on the variation symbol is included here as a reminder that this is a one form on phase space which need not be exact, the corresponding charge may not exist.

Table 1. A summary of the properties of the various charges and conservation laws reviewed in this section.

The boundary components can either be at a finite location, as for a black hole horizon, or can be asymptotic boundaries. In the latter case the manifold $M$ will be the unphysical spacetime of the conformal completion framework.

Two prototypical examples of setups we will want to consider are shown in figure 1 . In the first, the manifold $M$ is the domain of outer communications of a black hole formed in a gravitational collapse, and the boundary elements are future null infinity $\mathscr{I}^{+}$, past null infinity $\mathscr{I}^{-}$, and the future event horizon $\mathcal{H}^{+}$. In the second, the manifold is the domain of outer communications of an eternal black hole, and the boundary elements contain in addition the past event horizon $\mathcal{H}^{-}$. We will also be concerned with the boundaries $\mathcal{H}_{ \pm}^{+}$, $\mathscr{I}_{ \pm}^{+}$etc of these boundary elements, where $\mathcal{H}_{+}^{+}\left(\mathscr{I}_{+}^{+}\right)$is to be interpreted as the limit of cuts $\mathcal{S}$ of $\mathcal{H}^{+}\left(\mathscr{I}^{+}\right)$in the limit as $\mathcal{S}$ approaches future timelike infinity $i^{+}, \mathcal{H}_{-}^{+}$is the bifurcation two-sphere in the second case, and $\mathscr{I}_{-}^{+}$is the limit of cuts tending to spatial infinity $i^{0}$.

A crucial role in the formalism is the definition of a field configuration space $\mathscr{F}$ of fields $\phi$ on $M$. The fields are required to be smooth on $\mathcal{M}$ and to obey suitable bound- 


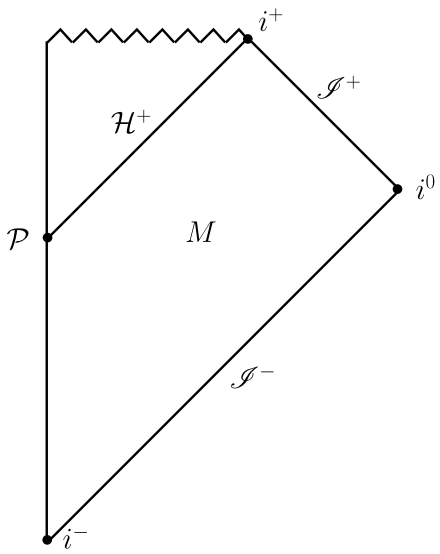

(a)

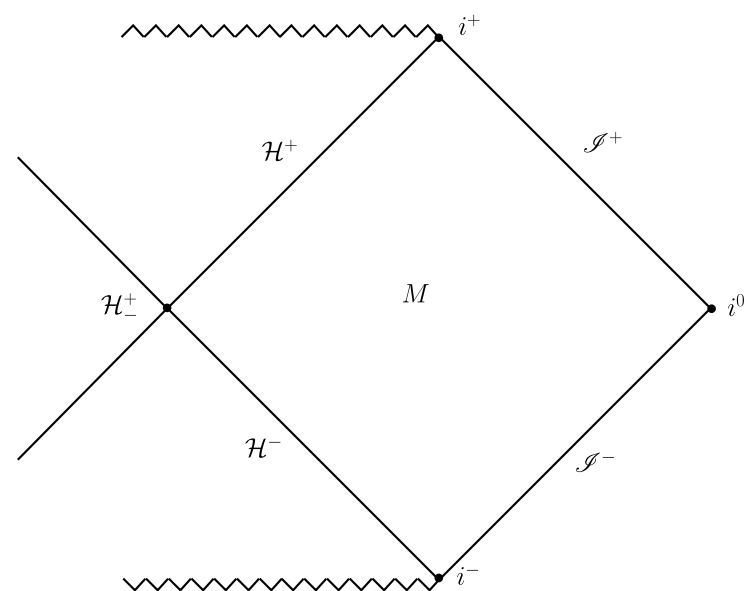

(b)

Figure 1. An illustration of two situations we will consider for the spacetime $M$. (a) $M$ is taken to be the domain of outer communications of a black hole formed in a gravitational collapse, with boundary elements $\mathscr{I}^{-}, \mathscr{I}^{+}$and $\mathcal{H}^{+}$. (b) $M$ is taken to be the domain of outer communications of an eternal black hole, with boundary elements $\mathscr{I}^{-}, \mathscr{I}^{+}, \mathcal{H}^{-}$and $\mathcal{H}^{+}$.

ary conditions at each boundary component $\mathcal{B}_{j}$ and at their intersections. A key goal of this paper is to determine appropriate boundary conditions for vacuum general relativity, for a boundary component which is a general null surface $\mathcal{N}$ at a finite location in spacetime. These boundary conditions should allow the computation of symmetries and charges. Boundary conditions that achieve this are specified in section 5.1 below.

\subsection{Definitions of currents}

We next review how conserved currents associated with spacetime symmetries are obtained from the Lagrangian [6]. We assume that the dynamics of the theory is obtained from a $d$-form Lagrangian

$$
\boldsymbol{L}=\boldsymbol{L}(\phi)
$$

which depends locally and covariantly on the fields $\phi$. Such a Lagrangian is independent of any "background fields". Under a field variation $\phi \rightarrow \phi+\delta \phi$ the variation of the Lagrangian can always be written as

$$
\delta \boldsymbol{L}=\boldsymbol{E}(\phi) \cdot \delta \phi+d \boldsymbol{\theta}(\phi, \delta \phi),
$$

where the tensor-valued $d$-form $\boldsymbol{E}(\phi)$ represents the equations of motion and represents contraction over any suppressed tensor indices. The $(d-1)$-form $\boldsymbol{\theta}(\phi, \delta \phi)$ is the presymplectic potential, which is locally and covariantly constructed out of $\phi$ and $\delta \phi$ and finitely many of their derivatives. The subspace of $\mathscr{F}$ satisfying the equations of motion $\boldsymbol{E}=0$ forms the covariant phase space $\overline{\mathscr{F}}$ of the theory.

Given two independent field variations $\delta_{1} \phi$ and $\delta_{2} \phi$ we define the presymplectic current

$$
\boldsymbol{\omega}\left(\phi, \delta_{1} \phi, \delta_{2} \phi\right)=\delta_{1} \boldsymbol{\theta}\left(\phi, \delta_{2} \phi\right)-\delta_{2} \boldsymbol{\theta}\left(\phi, \delta_{1} \phi\right)
$$


If $\phi$ satisfies the equations of motion and $\delta_{1} \phi$ and $\delta_{2} \phi$ satisfy the linearized equations of motion, then the presymplectic current is conserved,

$$
d \boldsymbol{\omega}=0 .
$$

We also define, for any vector field $\xi^{a}$ on spacetime, the Noether current $(d-1)$-form $\boldsymbol{j}_{\xi}$ by

$$
\boldsymbol{j}_{\xi}=\boldsymbol{\theta}\left(\phi, £_{\xi} \phi\right)-i_{\xi} \boldsymbol{L},
$$

where $i_{\xi}$ denotes contraction of the vector field with the differential form on the first index. It follows from eqs. (2.3) and (2.6) that $d \boldsymbol{j}_{\xi}=0$ on shell. For any local and covariant theory it can be shown that the Noether current can always be written in the form (see $[48,49]$ )

$$
\boldsymbol{j}_{\xi}=d \boldsymbol{Q}_{\xi}+\xi^{a} \boldsymbol{C}_{a}
$$

where $\boldsymbol{Q}_{\xi}(\phi)$ is the Noether charge $(d-2)$-form and $\boldsymbol{C}_{a}(\phi)$ are the constraints which vanish when the equations of motion hold. Taking a variation of the Noether current (2.6) and using eqs. (2.3), (2.4) and (2.7) we get for on-shell perturbations

$$
\boldsymbol{\omega}\left(\phi, \delta \phi, £_{\xi} \phi\right)=d\left[\delta \boldsymbol{Q}_{\xi}-i_{\xi} \boldsymbol{\theta}(\phi, \delta \phi)\right] .
$$

\subsection{Definition of presymplectic form on covariant phase space}

We next define the quantity

$$
\Omega_{\Sigma}\left(\phi, \delta_{1} \phi, \delta_{2} \phi\right)=\int_{\Sigma} \boldsymbol{\omega}\left(\phi, \delta_{1} \phi, \delta_{2} \phi\right),
$$

where $\Sigma$ is any hypersurface embedded in $M$. We would like to use the definition (2.9) specialized to a Cauchy surface $\Sigma$ to define the presymplectic form ${ }^{3}$ of the theory, a twoform on the covariant phase space $\overline{\mathscr{F}}$. There are a number of properties that we would like $\Omega_{\Sigma}$ to satisfy, some of which inform and restrict the definition of field configuration space $\mathscr{F}$. These properties are:

- Invariance under gauge transformations. One might expect that $\Omega_{\Sigma}\left(\phi, \delta_{1} \phi, \delta_{2} \phi\right)$ should be invariant under independent linearized diffeomorphisms acting on $\delta_{1} \phi$ and $\delta_{2} \phi$. This would require that $\Omega_{\Sigma}\left(\phi, \delta \phi, £_{\xi} \phi\right)=0$ for any $\phi \in \overline{\mathscr{F}}$ and for any vector fields $\xi^{a}$ and variations $\delta \phi$ for which $\delta \phi$ and $£_{\xi} \phi$ are tangent to $\overline{\mathscr{F}}$. However, this is not true in general. Instead, from eqs. (2.8) and (2.9) we have that, on shell, for a Cauchy surface $\Sigma$,

$$
\Omega_{\Sigma}\left(\phi, \delta \phi, £_{\xi} \phi\right)=\int_{\partial \Sigma} \delta \boldsymbol{Q}_{\xi}(\phi)-i_{\xi} \boldsymbol{\theta}(\phi, \delta \phi)
$$

where $\partial \Sigma$ is the boundary of $\Sigma$, a $d-2$-surface in $\partial M$. This quantity vanishes for vector fields whose support lies in the interior of $M$, but not in general for vector

\footnotetext{
${ }^{3}$ The presymplectic form $\Omega_{\Sigma}$ is usually degenerate. One can factor the configuration space $\mathscr{F}$ by the orbits of the degeneracy subspaces of $\Omega_{\Sigma}$ to obtain a phase space $\Gamma$ on which there exists a nondegenerate symplectic form [43]. However this will not be needed in what follows.
} 
fields which are nonzero on the boundary $\partial M$. As is well known, the fact that these diffeomorphisms do not correspond to degeneracy directions of the presymplectic form reflects the fact that the corresponding degrees of freedom are physical and not gauge. ${ }^{4}$

- Finiteness at asymptotic boundaries. The definition (2.9) is invariant under local deformations of the hypersurface $\Sigma$ when on-shell, from eq. (2.5). We would like the presymplectic form $(2.9)$ to have a well defined limit as $\Sigma$ approaches $\mathscr{I}^{+}$or $\mathscr{I}^{-}$, which will be true if the presymplectic current $\boldsymbol{\omega}$ has a well defined limit on those boundaries. Boundary conditions at $\mathscr{I}^{+}$and $\mathscr{I}^{-}$that are sufficient to ensure this are given by Wald and Zoupas [6] (see their footnote 16). These boundary conditions supplement the standard definition of asymptotic flatness at null infinity [50] by specializing the gauge, ${ }^{5}$ and are necessary for $\boldsymbol{\omega}$ to have a finite limit. In the context of null boundaries at finite locations discussed in this paper, we will also for convenience specialize the gauge at the boundary (see section 5.1 below). There is a tension between gauge specializations at the boundary and the fact that some of the diffeomorphism degrees of freedom on the boundary become physical: one does not want to restrict physical degrees of freedom in the definition of the field configuration space $\mathscr{F}$. A general strategy for dealing with this tension is discussed in section 5.1 below.

- Independence of choice of Cauchy surface. In order for $\Omega_{\Sigma}$ to define a presymplectic form on the covariant phase space $\overline{\mathscr{F}}$, one would like it to be independent of the choice of Cauchy surface $\Sigma$. While the integral (2.9) is invariant under local deformations of the hypersurface $\Sigma$, when one takes a limit to the boundary of spacetime there can nonzero contributions to the limiting integral from "corners" of the spacetime where boundary elements intersect, such as spatial infinity $i^{0}$. One would like to specialize the definition of the field configuration space $\mathscr{F}$ to eliminate such contributions. This issue is closely related to the question of the validity of the global conservation laws discussed in section 7 below.

\subsection{Global charges that generate boundary symmetries}

We now turn to a discussion of spacetime symmetries, which we will also call boundary symmetries since only the action of the symmetry near the boundary $\partial M$ of spacetime will be important [6]. Infinitesimal diffeomorphisms are parametrized by vector fields $\xi^{a}$ on $M$, under which fields transform as $\phi \rightarrow \phi+\delta \phi$, where

$$
\delta \phi=£_{\xi} \phi
$$

Fix attention on one component $\mathcal{B}_{j}$ of the boundary $\partial M$. We denote by $G_{j}$ the set of smooth vector fields $\xi^{a}$ on $M$ such that the diffeomorphism generated by $\xi^{a}$ preserves the boundary $\partial M$, and such that for any solution $\phi \in \overline{\mathscr{F}}$, the transformed solution $\phi+£_{\xi} \phi$

\footnotetext{
${ }^{4}$ One can choose to restore full diffeomorphism invariance by performing the Stueckelberg trick and introducing new physical degrees of freedom on the boundary, so-called edge modes [34, 35, 46].

${ }^{5}$ Here by gauge we mean both diffeomorphism freedom and choice of conformal factor.
} 
satisfies any boundary conditions at $\mathcal{B}_{j}$ imposed on fields in $\mathscr{F}$, to linear order in $\xi^{a}$. We will call such a vector field a representative of an infinitesimal boundary symmetry at $\mathcal{B}_{j}$. We also define $G$ to be the set of smooth vector fields whose diffeomorphisms preserve $\partial M$ and map $\overline{\mathscr{F}}$ to $\overline{\mathscr{F}}$ under pullback, which we call representatives of infinitesimal boundary symmetries. ${ }^{6}$

Consider now a representative of an infinitesimal boundary symmetry $\xi^{a}$. We would like to construct a charge $\mathcal{Q}_{\xi}$, a function on $\mathscr{F}$, which generates the boundary symmetry (2.11). This means that $\mathcal{Q}_{\xi}$ should satisfy [6]

$$
\delta \mathcal{Q}_{\xi}=\Omega_{\Sigma}\left(\phi, \delta \phi, £_{\xi} \phi\right)=\int_{\Sigma} \boldsymbol{\omega}\left(\phi, \delta \phi, £_{\xi} \phi\right)
$$

for all $\phi \in \overline{\mathscr{F}}$ and for all $\delta \phi, £_{\xi} \phi$ tangent to $\mathscr{F}$, where $\Sigma$ is a Cauchy surface. The charge $\mathcal{Q}_{\xi}$ can be interpreted as a Hamiltonian ${ }^{7}$ in the special case when $\xi$ is a timelike vector field. We call the charges (2.12) global charges since they are obtained by an integral over a complete Cauchy surface and so involve all the degrees of freedom in the theory, in contrast to the localized charges discussed in section 2.6 below.

We next discuss the conditions under which the boundary symmetry generator charge $\mathcal{Q}_{\xi}$ will exist. Since eq. (2.12) is attempting to define an exact one-form on field configuration space, the right hand side should be a closed one-form. It follows from eq. (2.8) that the variation of the charge is a surface term on-shell:

$$
\delta \mathcal{Q}_{\xi}=\int_{\partial \Sigma} \delta \boldsymbol{Q}_{\xi}-i_{\xi} \boldsymbol{\theta}(\phi, \delta \phi)
$$

If the boundary $\partial \Sigma$ consists of a number of disconnected components $\mathcal{S}_{j}$, then $\delta \mathcal{Q}_{\xi}=$ $\sum_{j} \delta \mathcal{Q}_{\xi, j}$ where

$$
\delta \mathcal{Q}_{\xi, j}=\int_{\mathcal{S}_{j}} \delta \boldsymbol{Q}_{\xi}-i_{\xi} \boldsymbol{\theta}(\phi, \delta \phi)
$$

Taking a second variation and using the definition (2.4) of the presymplectic current gives $[6]$

$$
0=\left(\delta_{1} \delta_{2}-\delta_{2} \delta_{1}\right) \mathcal{Q}_{\xi}=-\int_{\partial \Sigma} i_{\xi} \boldsymbol{\omega}\left(\phi, \delta_{1} \phi, \delta_{2} \phi\right)
$$

The quantity (2.15) must vanish for all $\delta_{1} \phi$ and $\delta_{2} \phi$ tangent to $\overline{\mathscr{F}}$ in order for the charge $\mathcal{Q}_{\xi}$ to exist. When it does vanish, ${ }^{8}$ the definition (2.12) determines the charge on $\overline{\mathscr{F}}$ up

\footnotetext{
${ }^{6}$ The set $G$ will generally be a proper subset of $\cap_{j} G_{j}$, because of boundary conditions imposed at intersections of boundary elements in the definition of $\mathscr{F}$ (for example continuity at a bifurcation twosphere in an eternal black hole spacetime). See section 7 below for further discussion.

${ }^{7}$ Here we depart slightly from the terminology used by Wald and Zoupas [6], who call all such charges Hamiltonians and denote them by $H_{\xi}$. The definition of Wald and Zoupas — their eq. (8) — is also more general since they do not impose that $\Sigma$ be a Cauchy surface. We will return to this generalization in section 2.6 below.

${ }^{8}$ Note that in general the second term in eq. (2.13) can give a nonvanishing contribution, so that the charge differs from the Noether charge, even when the obstruction (2.15) vanishes. This occurs for example for ADM charges at spatial infinity [51].
} 
to constants of integration on phase space, which can be specified by demanding that the charge vanish on a reference solution on each connected component of $\overline{\mathscr{F}}[6]$. This prescription is discussed in more detail in the more general context of localized charges in section 2.6 below.

In all cases that we are aware of, the condition (2.15) is satisfied whenever $\Sigma$ is taken to be a Cauchy surface, as here. While we are not aware of a general proof, there is a physical argument indicating that the condition should be satisfied: a non-vanishing pullback of the symplectic current to $\partial \Sigma$ in (2.15) reflects an interaction between degrees of freedom that have been included in the integral (2.12) and those that have been excluded, and Cauchy surfaces include all of the degrees of freedom. Some examples of cases where the condition (2.15) is satisfied include:

- Spacetimes in general relativity that are asymptotically flat at spatial infinity $i^{0}$ and vacuum in a neighborhood of $i^{0}$, and spacelike Cauchy surfaces $\Sigma$ that extend to $i^{0}$. In this case the presymplectic current extends continuously to the boundary but has vanishing pullback there [6].

- Asymptotically flat spacetimes in vacuum general relativity with no horizons, with $\Sigma$ taken to be future null infinity $\mathscr{I}^{+}$, with certain fall off conditions on the News tensor. Consider the integrand in the obstruction (2.15), in the limit where the cut $\mathcal{S}$ of $\mathscr{I}^{+}$approaches $\mathscr{I}_{+}^{+}$or $\mathscr{I}_{-}^{+}$, i.e., $i^{+}$or $i^{0}$. Denoting affine parameter by $u$, the integrand is given by eq. (72) of [6] and scales like a symmetry generator $\sim u$, times a shear tensor $\sim u^{0}$, times a News tensor. Hence if the News tensor decays faster than $1 /|u|$ as $|u| \rightarrow \infty$ the result vanishes:

$$
\int_{\mathscr{I}_{ \pm}^{+}} i_{\xi} \omega=0
$$

In the Christodoulou-Klainerman class of spacetimes [52] the News decays like $|u|^{-3 / 2}$.

- In the previous example, if the spacetime contains in addition a future event horizon $\mathcal{H}^{+}$, then the Cauchy surface can be taken to be $\mathcal{H}^{+} \cup \mathscr{I}^{+}$and the integral (2.12) will contain contributions from both $\mathcal{H}^{+}$and $\mathscr{I}^{+}$:

$$
\delta \mathcal{Q}_{\xi}=\int_{\mathcal{H}^{+}} \boldsymbol{\omega}\left(\phi, \delta \phi, £_{\xi} \phi\right)+\int_{\mathscr{I}+} \boldsymbol{\omega}\left(\phi, \delta \phi, £_{\xi} \phi\right) .
$$

Here the first term will depend only on the limiting form of the symmetry $\xi^{a}$ near $\mathcal{H}^{+}$, and the second term only on the limiting form near $\mathscr{I}^{+}$. The integrability analysis described above can be applied to each of these terms separately. In appendix G we show that the condition (2.15) is satisfied for the integral over $\mathcal{H}^{+}$under certain conditions (as well as for the integral over $\mathscr{I}^{+}$).

To summarize this discussion, the definition (2.12) should be sufficient to compute global charges $\mathcal{Q}_{\xi}$ that generate boundary symmetries when $\Sigma$ is a Cauchy surface. See the review article by Strominger [7] for several specific calculations of charges of this type. In section 6.3 below we will compute explicitly the contribution to such charges from boundary elements that are null surfaces at a finite location in spacetime, and in section 7 we will discuss global conservation laws that are satisfied by global charges $\mathcal{Q}_{\xi}$. 


\subsection{Boundary symmetry algebras of linearized diffeomorphisms}

We next discuss the symmetry algebras associated with each component $\mathcal{B}_{j}$ of the boundary $\partial M$ of spacetime. These are obtained from the set $G_{j}$ of representatives of infinitesimal boundary symmetries at $\mathcal{B}_{j}$ by modding out the trivial representatives whose charges (2.13) vanish [6]. Specifically, we define an equivalence relation on representatives $\xi^{a}$ by

$$
\xi^{a} \sim \xi^{\prime a} \quad \text { if } \quad \xi^{a} \widehat{=} \xi^{\prime a} \text { and } \int_{\mathcal{S}}\left(\delta \boldsymbol{Q}_{\xi}-i_{\xi} \boldsymbol{\theta}\right)=\int_{\mathcal{S}}\left(\delta \boldsymbol{Q}_{\xi^{\prime}}-i_{\xi^{\prime}} \boldsymbol{\theta}\right)
$$

Here the notation $\widehat{=}$ means equal when evaluated on $\mathcal{B}_{j}$, and the integrals must coincide for all $\phi \in \overline{\mathscr{F}}$ and $\delta \phi$ tangent to $\overline{\mathscr{F}}$ and for all cross sections $\mathcal{S}$ of $\mathcal{B}_{j}$. We define the symmetry algebra

$$
\mathfrak{g}_{j}=G_{j} / \sim,
$$

which for example gives the BMS algebra at null infinity [6]. In section 5.2 below we will derive the corresponding symmetry algebra for a null surface at a finite location.

We similarly define the global symmetry algebra $\mathfrak{g}=G / \sim$, where now the equivalence relation is defined by imposing eq. (2.18) at all cross sections $\mathcal{S}$ of all boundary components $\mathcal{B}_{j}$. In general $\mathfrak{g}$ will be a proper subalgebra of the direct sum algebra

$$
\bigoplus_{j} \mathfrak{g}_{j}
$$

because of boundary conditions imposed at the intersections of boundary components in the definition of $\mathscr{F}$, cf. the discussions in section 2.4 above and 7 below.

\subsection{Localized (Wald-Zoupas) charges, fluxes and conservation laws}

We now turn to a discussion of a different type of charge which we call localized charges, whose physical interpretation is roughly the amount of charge in a subset of the degrees of freedom of the theory. Studies of this type of charge have a long history in general relativity. For example, there have been many attempts made to define the total mass in a finite region of space, using various notions of quasilocal mass [53], but no natural and generally accepted definition has emerged. On the other hand, as is well known, the total amount of 4-momentum ${ }^{9}$ radiated through any finite region of future null infinity is uniquely defined [8, 9]. Wald and Zoupas [6] give a very general prescription for defining localized charges of this type at a boundary of spacetime, for any diffeomorphism invariant theory and for a large class of boundary conditions. They show that their general prescription gives the conventional results $[8,9]$ for BMS charges at null infinity. In this subsection we review and specialize slightly their general construction, and in section 6.4 below we apply it to compute localized charges at a spacetime boundary consisting of a null surface at a finite location.

One trivial kind of localization was already encountered in section 2.4 above. In the example (2.17), the charge variation $\delta \mathcal{Q}_{\xi}$ was expressed as a sum of an integral over the

\footnotetext{
${ }^{9}$ Or more generally any BMS charge.
} 
future event horizon $\mathcal{H}^{+}$and an integral over future null infinity $\mathscr{I}^{+}$, each of which individually satisfies the integrability condition (2.15). Here we want to go further and consider charges localized to subregions of boundary components.

Consider a region $\Delta \mathcal{B}_{j}$ of a boundary $\mathcal{B}_{j}$ whose boundary consists of two crosssections $\mathcal{S}$ and $\mathcal{S}^{\prime}$, and a representative $\xi^{a}$ of an infinitesimal boundary symmetry at $\mathcal{B}_{j}$. Given a solution $\phi \in \overline{\mathscr{F}}$, we would like to define an exact 3 -form $d \mathcal{Q}_{\xi}^{\text {loc }}$ on $\mathcal{B}_{j}$ for which the charge in the region $\Delta \mathcal{B}_{j}$ is

$$
\int_{\Delta \mathcal{B}_{j}} d \mathcal{Q}_{\xi}^{\text {loc }}=\mathcal{Q}_{\xi}^{\text {loc }}\left(\mathcal{S}^{\prime}\right)-\mathcal{Q}_{\xi}^{\text {loc }}(\mathcal{S})
$$

where

$$
\mathcal{Q}_{\xi}^{\text {loc }}(\mathcal{S})=\int_{\mathcal{S}} \mathcal{Q}_{\xi}^{\text {loc }}
$$

is the charge at crosssection $\mathcal{S}$. We will call the quantity (2.22) a localized or Wald-Zoupas charge. The prototypical example of a quantity like this is the Bondi mass at a cross section $\mathcal{S}$ of $\mathscr{I}^{+}$, which is the total mass of the spacetime minus the mass radiated up to $\mathcal{S}$. In section 6.4 we will define a similar quantity at cuts of a null boundary, which for a future event horizon will be the total charge at the bifurcation twosphere of the black hole (if any) plus the total charge accreted by the black hole up to the cut $\mathcal{S}{ }^{10}$

In the limit $\Delta \mathcal{B}_{j} \rightarrow \mathcal{B}_{j}$, the quantity (2.21) should reduce to the contribution from $\mathcal{B}_{j}$ to the global charge $\mathcal{Q}_{\xi}$. A natural candidate prescription for defining a $d-2$-form $\mathcal{Q}_{\xi}^{\text {loc }}$ that would achieve this is given by taking $\Sigma=\Delta \mathcal{B}_{j}$ in the definition (2.12), or, from eqs. (2.13) and (2.21),

$$
\delta \mathcal{Q}_{\xi}^{\text {loc }}=\delta \boldsymbol{Q}_{\xi}-i_{\xi} \boldsymbol{\theta}
$$

However, the corresponding charge (2.22) will generally not exist because of the obstruction (2.15). One would like to modify the right hand side of eq. (2.23) in such a way as to remove this obstruction, without changing the integral on the left hand side of (2.21) in the limit $\Delta \mathcal{B}_{j} \rightarrow \mathcal{B}_{j}$. One would also like to find a natural prescription for this modification that yields unique charges. One could then interpret eq. (2.21) as a localized conservation law, which equates a flux through a region of $\mathcal{B}_{j}$ with the difference between the charges at the two crosssections. (A distinct kind of global conservation law involving global charges $\mathcal{Q}_{\xi}$ is discussed in section 7 below.)

Wald and Zoupas [6] suggested a prescription of this kind that gives unique answers under certain conditions, which can be summarized as follows (we omit some subtleties related to taking the limit to asymptotic boundaries that will not be relevant for our application):

1. Compute the pullback $\boldsymbol{\omega}\left(\bar{\phi}, \overline{\delta_{1} \phi}, \overline{\delta_{2} \phi}\right)$ to the boundary component $\mathcal{B}_{j}$ of the presymplectic current $\boldsymbol{\omega}\left(\phi, \delta_{1} \phi, \delta_{2} \phi\right)$. Here the barred fields are the dynamical fields on the boundary induced by the solution $\phi \in \overline{\mathscr{F}}$ and linearized solutions $\delta_{1} \phi, \delta_{2} \phi$ tangent to $\overline{\mathscr{F}}$, obtained by taking pullbacks of these fields (and possibly their derivatives) to the boundary.

\footnotetext{
${ }^{10}$ Our orientation convention is such that $(2.21)$ is valid at $\mathscr{I}^{+}$when $\mathcal{S}$ is to the future of $\mathcal{S}^{\prime}$, while at a future event horizon $\mathcal{H}^{+}$it is valid when $\mathcal{S}^{\prime}$ is to the future of $\mathcal{S}$.
} 
2. Choose a presymplectic potential $\Theta(\bar{\phi}, \overline{\delta \phi})$ on $\mathcal{B}_{j}$ for the pullback $\boldsymbol{\omega}$, that is, a $d-1$ form which satisfies

$$
\boldsymbol{\omega}\left(\bar{\phi}, \overline{\delta_{1} \phi}, \overline{\delta_{2} \phi}\right)=\delta_{1} \boldsymbol{\Theta}\left(\bar{\phi}, \overline{\delta_{2} \phi}\right)-\delta_{2} \Theta\left(\bar{\phi}, \overline{\delta_{1} \phi}\right)
$$

We require that the dependence of $\boldsymbol{\Theta}$ on the dynamical fields on the boundary, as well as the dependence on fields in any universal background structure on $\mathcal{B}_{j}$ inherent in the definition of the field configuration space $\mathscr{F}$, be local and covariant. ${ }^{11}$ (See sections 4 and 5 for more details on universal background structures.)

3. Add the term $i_{\xi} \Theta$ to the right hand side of eq. (2.23), thus giving from eq. (2.22) the following formula for the variation of the localized charge:

$$
\delta \mathcal{Q}_{\xi}^{\text {loc }}(\mathcal{S})=\int_{\mathcal{S}} \delta \mathcal{Q}_{\xi}^{\text {loc }}=\int_{\mathcal{S}} \delta \boldsymbol{Q}_{\xi}-i_{\xi} \boldsymbol{\theta}+i_{\xi} \boldsymbol{\Theta}
$$

4. Now repeating the computation that led to eq. (2.15) shows that the obstruction now vanishes. The definition $(2.25)$ therefore determines the charge $\mathcal{Q}_{\xi}^{\text {loc }}(\mathcal{S})$ on $\overline{\mathscr{F}}$ up to constants of integration on phase space, which can be specified by demanding that the charges vanish on a reference solution ${ }^{12} \phi_{0}$ on each connected component of $\overline{\mathscr{F}}$,

$$
\left.\mathcal{Q}_{\xi}^{\text {loc }}(\mathcal{S})\right|_{\phi=\phi_{0}}=0
$$

for all symmetry representatives $\xi^{a}$ and cuts $\mathcal{S}[6]$.

5. In order to reduce the non-uniqueness in the boundary presymplectic potential $\Theta$, we impose the requirement that

$$
\Theta(\bar{\phi}, \overline{\delta \phi})=0
$$

for all $\overline{\delta \phi}$ whenever $\bar{\phi}$ is stationary ${ }^{13}$ at $\mathcal{B}_{j}$. We also impose that the reference solution $\phi_{0}$ be stationary at $\mathcal{B}_{j}$.

The motivation for the fifth requirement is as follows [6]. It is natural on physical grounds to demand that the flux $d \mathcal{Q}_{\xi}^{\text {loc }}$ vanish for solutions which are stationary at the

\footnotetext{
${ }^{11}$ What this means is as follows. The presympletic potential $\Theta$ depends on a field configuration $\phi$, its variation $\delta \phi$, a universal background structure on $\mathcal{B}_{j}$ which we denote by $\mathfrak{p}$, and on the boundary $\mathcal{B}_{j}$ : $\boldsymbol{\Theta}=\boldsymbol{\Theta}\left(\phi, \delta \phi, \mathfrak{p}, \mathcal{B}_{j}\right)$. Locality and covariance requires that for any diffeomorphism $\psi: M \rightarrow M$,

$$
\psi_{*} \Theta\left(\phi, \delta \phi, \mathfrak{p}, \mathcal{B}_{j}\right)=\boldsymbol{\Theta}\left(\psi_{*} \phi, \psi_{*} \delta \phi, \psi_{*} \mathfrak{p}, \psi^{-1}\left(\mathcal{B}_{j}\right)\right),
$$

where $\psi_{*}$ is the pullback. If we specialize to diffeomorphisms which preserve the boundary, $\psi^{-1}\left(\mathcal{B}_{j}\right)=$ $\mathcal{B}_{j}$, and the universal background structure on the boundary, $\psi_{*} \mathfrak{p}=\mathfrak{p}$, then $\psi_{*} \Theta\left(\phi, \delta \phi, \mathfrak{p}, \mathcal{B}_{j}\right)=$ $\boldsymbol{\Theta}\left(\psi_{*} \phi, \psi_{*} \delta \phi, \mathfrak{p}, \mathcal{B}_{j}\right)$.

${ }^{12}$ And on all solutions related to $\phi_{0}$ by linearized diffeomorphisms. See appendix E for further discussion of this point.

${ }^{13}$ By "stationary at $\mathcal{B}_{j}$ " we mean that there exists a representative $\tau^{a}$ of an infinitesimal boundary symmetry at $\mathcal{B}_{j}$ which is timelike and satisfies the Killing equation on $\mathcal{B}_{j}$ and to first order in deviations off $\mathcal{B}_{j}$. This is a weaker notion than used in [6].
} 
boundary $\mathcal{B}_{j}$. Taking the exterior derivative of the integrand in eq. (2.25) and using eq. (2.8) and the fact that $d$ and $\delta$ commute we get

$$
\begin{aligned}
\delta d \mathcal{Q}_{\xi}^{\mathrm{loc}} & =\boldsymbol{\omega}\left(\phi ; \delta \phi, £_{\xi} \phi\right)+d\left[i_{\xi} \boldsymbol{\Theta}(\phi ; \delta \phi)\right]=\boldsymbol{\omega}\left(\phi ; \delta \phi, £_{\xi} \phi\right)+£_{\xi} \boldsymbol{\Theta}(\phi ; \delta \phi) \\
& =\delta \boldsymbol{\Theta}\left(\phi ; £_{\xi} \phi\right) .
\end{aligned}
$$

To integrate this on $\overline{\mathscr{F}}$, note that $\mathcal{Q}_{\xi}^{\text {loc }}$ must vanish identically on $\phi_{0}$ by eq. (2.26), while $\boldsymbol{\Theta}\left(\phi_{0}, \delta \phi\right)$ vanishes by eq. (2.27). Thus we obtain

$$
d \mathcal{Q}_{\xi}^{\text {loc }}=\boldsymbol{\Theta}\left(\phi ; £_{\xi} \phi\right),
$$

and so the flux vanishes identically on stationary solutions as desired, by eq. (2.27).

A useful method of parameterizing choices of $\Theta$ that automatically satisfy all the requirements apart from the stationary requirement (2.27) is

$$
\Theta=\boldsymbol{\theta}-\delta \boldsymbol{\alpha}
$$

where the first term on the right hand side is the pullback of the presymplectic potential $\boldsymbol{\theta}$, and $\boldsymbol{\alpha}$ is some $d-1$-form on $\mathcal{B}_{j}$ constructed from $\bar{\phi}$. Inserting this into eq. (2.25), integrating in the covariant phase space $\overline{\mathscr{F}}$ and using eq. (2.26) now gives

$$
\mathcal{Q}_{\xi}^{\mathrm{loc}}(\mathcal{S})=\int_{\mathcal{S}} \boldsymbol{Q}_{\xi}-i_{\xi} \boldsymbol{\alpha}
$$

if the right hand side vanishes on the reference solution $\phi=\phi_{0}$. In section 6 we will show that at a null boundary for vacuum general relativity one can choose $\boldsymbol{\alpha}$ so that $\boldsymbol{\Theta}$ satisfies the criteria outlined above, with the definition of stationary of footnote 13 replaced by the weaker notion of shear free and expansion free.

Finally, the global charges $\mathcal{Q}_{\xi}$ discussed in section 2.4 above can often be written in terms of the localized charges $\mathcal{Q}_{\xi}^{\text {loc }}(\mathcal{S})$ discussed here, specialized to specific cross sections $\mathcal{S}$ :

$$
\mathcal{Q}_{\xi}=\sum_{j} \mathcal{Q}_{\xi}^{\text {loc }}\left(\mathcal{S}_{j}\right)=\sum_{j} \int_{\mathcal{S}_{j}} \mathcal{Q}_{\xi}^{\text {loc }}
$$

where the boundary $\partial \Sigma$ of a Cauchy surface $\Sigma$ is a union $\partial \Sigma=\cup_{j} \mathcal{S}_{j}$ of disconnected components $\mathcal{S}_{j}$. The relation (2.32) will hold when the correction term $i_{\xi} \boldsymbol{\Theta}$ in the definition (2.25) of the localized charge vanishes on $\partial \Sigma$, from the definition (2.13), if the same reference solution is used for the localized and global charges. We expect the correction term $i_{\xi} \Theta$ to generically vanish on $\partial \Sigma$ when $\Sigma$ is a Cauchy surface. Some examples where this occurs are:

- At future null infinity $\mathscr{I}^{+}$, the correction term $i_{\xi} \Theta$ is proportional to the generator $\xi^{a}$ times the News tensor (eq. (73) of [6]). Letting $u$ denote an affine parameter along $\mathscr{I}^{+}$, the generator scales as $\sim|u|$ as $u \rightarrow \pm \infty$, and so if the News tensor decays faster than $1 /|u|$, the contributions from the boundaries $\mathscr{I}_{ \pm}^{+}$of $\mathscr{I}^{+}$will vanish [cf. the discussion before eq. (2.17) above]. 
- For a future event horizon $\mathcal{H}^{+}$, we show in appendix $\mathrm{G}$ that the contribution to the correction term from the future boundary $\mathcal{H}_{+}^{+}$(the limit to $i^{+}$) of the horizon vanishes, if the shear obeys a suitable decay condition near $\mathcal{H}_{+}^{+}$. We also show that the contribution from a bifurcation two-sphere $\mathcal{H}_{-}^{+}$vanishes.

Explicit expressions for $\mathcal{Q}_{\xi}^{\text {loc }}(\mathcal{S})$ for cross sections $\mathcal{S}$ of future null infinity $\mathscr{I}^{+}$are given in eqs. (92) and (98) of Wald and Zoupas [6], and specialized to Bondi coordinates in eq. $(3.5)$ of ref. [54]. For cross sections of an arbitrary null surface, our result for $\mathcal{Q}_{\xi}^{\text {loc }}(\mathcal{S})$ is given in eq. (6.27) below.

\subsection{Potential ambiguities in global and localized charges}

We next discuss some ambiguities that can arise in the definitions and constructions outlined above of global and localized charges $[6,46,55]$. Wald and Zoupas show that these ambiguities can be resolved in vacuum general relativity at future null infinity. We will similarly argue that they can be resolved at null boundaries at finite locations. However, they may be significant for other theories or at other types of boundary.

First, the definition (2.3) of the presymplectic potential $\boldsymbol{\theta}$ determines it up to a closed form. Since we require that $\boldsymbol{\theta}$ be local and covariant this closed form is also exact [56]. The corresponding ambiguities are

$$
\begin{aligned}
\boldsymbol{\theta}(\phi, \delta \phi) & \rightarrow \boldsymbol{\theta}(\phi, \delta \phi)+d \boldsymbol{Y}(\phi, \delta \phi) \\
\boldsymbol{\omega}\left(\phi, \delta_{1} \phi, \delta_{2} \phi\right) & \rightarrow \boldsymbol{\omega}\left(\phi, \delta_{1} \phi, \delta_{2} \phi\right)+d\left[\delta_{1} \boldsymbol{Y}\left(\phi, \delta_{2} \phi\right)-\delta_{2} \boldsymbol{Y}\left(\phi, \delta_{1} \phi\right)\right]
\end{aligned}
$$

for some $(d-2)$-form $\boldsymbol{Y}$. These give rise to the following transformations of the presymplectic potential $\Theta$ and of the localized charge $\mathcal{Q}_{\xi}^{\text {loc }}(\mathcal{S})$ :

$$
\begin{aligned}
\boldsymbol{\Theta}(\phi, \delta \phi) & \rightarrow \boldsymbol{\Theta}(\phi, \delta \phi)+d \boldsymbol{Y}(\phi, \delta \phi), \\
\mathcal{Q}_{\xi}^{\mathrm{loc}}(\mathcal{S}) & \rightarrow \mathcal{Q}_{\xi}^{\mathrm{loc}}(\mathcal{S})+\int_{\mathcal{S}} \boldsymbol{Y}\left(\phi, £_{\xi} \phi\right) .
\end{aligned}
$$

One can demand that the maximum number of derivatives of the fields $\phi$ or their variations $\delta \phi$ in the $(d-2)$-form $\boldsymbol{Y}$ be two less then the number of derivatives appearing in the Lagrangian. This requirement is in some sense natural, since otherwise the number of derivatives in $\boldsymbol{\theta}$ from eq. (2.33a) exceeds what one would naively expect from eq. (2.3). In section 6.1 below we argue that this requirement eliminates the ambiguity (2.33) for vacuum general relativity.

Second, the definition (2.24) of the presymplectic potential $\Theta$ determines it only up a transformation of the form

$$
\boldsymbol{\Theta}(\phi, \delta \phi) \rightarrow \boldsymbol{\Theta}(\phi, \delta \phi)+\delta \boldsymbol{W}(\phi),
$$

where $\boldsymbol{W}$ is constructed locally and covariantly from the field $\phi$ and from any universal background structure on $\mathcal{B}_{j}$. The localized charge transforms under this ambiguity as

$$
\mathcal{Q}_{\xi}^{\text {loc }}(\mathcal{S}) \rightarrow \mathcal{Q}_{\xi}^{\text {loc }}(\mathcal{S})+\int_{\mathcal{S}} i_{\xi} \boldsymbol{W}
$$


From the requirement (2.27) it follows that $\delta \boldsymbol{W}(\phi)$ must vanish for all solutions $\phi$ that are stationary at $\mathcal{B}_{j}$, and for all linearized solutions $\delta \phi$. If one additionally assumes that $\boldsymbol{W}$ depends analytically on the fields, it follows that $\boldsymbol{W}=0$ at future null infinity $\mathscr{I}^{+}$in vacuum general relativity [6]. We give a similar argument in section 6.4 below to show that the ambiguity $\boldsymbol{W}$ vanishes at finite null surfaces, if we assume that the maximum number of derivatives appearing in $\boldsymbol{W}$ is one less than the number of derivatives appearing in the Lagrangian.

Third, one can redefine the Lagrangian by an exact form, $\boldsymbol{L} \rightarrow \boldsymbol{L}+d \boldsymbol{K}$, without changing the equations of motion of the theory. The corresponding transformations of the presymplectic potential $\boldsymbol{\theta}$, presymplectic current $\boldsymbol{\omega}$, Noether charge $d-2$-form $\boldsymbol{Q}_{\xi}$ and the integrands $\delta \boldsymbol{Q}_{\xi}-i_{\xi} \boldsymbol{\theta}$ and $d \mathcal{Q}_{\xi}^{\text {loc }}$ of the symmetry generator charge (2.13) and localized charge (2.25) are given by

$$
\begin{aligned}
\boldsymbol{\theta}(\phi, \delta \phi) & \rightarrow \boldsymbol{\theta}(\phi, \delta \phi)+\delta \boldsymbol{K}(\phi), \\
\boldsymbol{\omega}\left(\phi, \delta_{1} \phi, \delta_{2} \phi\right) & \rightarrow \boldsymbol{\omega}\left(\phi, \delta_{1} \phi, \delta_{2} \phi\right), \\
\boldsymbol{Q}_{\xi}(\phi) & \rightarrow \boldsymbol{Q}_{\xi}(\phi)+i_{\xi} \boldsymbol{K}(\phi), \\
\delta \boldsymbol{Q}_{\xi}-i_{\xi} \boldsymbol{\theta} & \rightarrow \delta \boldsymbol{Q}_{\xi}-i_{\xi} \boldsymbol{\theta}, \\
\mathcal{Q}_{\xi}^{\text {loc }}(\phi) & \rightarrow \mathcal{Q}_{\xi}^{\text {loc }}(\phi) .
\end{aligned}
$$

While this transformation does affect the Noether charge, it does not affect the symmetry generator charge $\mathcal{Q}_{\xi}$ and localized charge $\mathcal{Q}_{\xi}^{\text {loc }}$ that are of the most interest for this paper.

\section{Review of the local geometry of null hypersurfaces}

\subsection{Foundations}

In this section we review the local geometry of null hypersurfaces $[57,58]$, in order to fix our notations and conventions. For the remainder of the paper we specialize to $3+1$ spacetime dimensions. Suppose we are given a spacetime $\left(M, g_{a b}\right)$, and a null hypersurface $\mathcal{N}$ in $M$ whose topology is $\mathcal{Z} \times \mathbb{R}$ for some base space $\mathcal{Z}$. We denote by $\ell_{a}$ a choice of future directed, null normal to the surface $\mathcal{N}$. This normal is not unique but can be rescaled according to

$$
\ell_{a} \rightarrow e^{\sigma} \ell_{a}
$$

where $\sigma$ is any smooth function on $\mathcal{N}$. We define the non-affinity $\kappa$, a function on $\mathcal{N}$, by

$$
\ell^{a} \nabla_{a} \ell^{b} \widehat{=} \kappa l^{b} .
$$

As a reminder here we are using $\widehat{=}$ to mean equality when restricted to $\mathcal{N}$. The non-affinity transforms under the rescaling (3.1) as

$$
\kappa \rightarrow e^{\sigma}\left(\kappa+£_{\ell} \sigma\right)
$$

We will adopt the terminology that any quantity $f$ which transforms under the transformation (3.1) as

$$
f \rightarrow e^{-n \sigma} f
$$

has scaling weight $n$. 
We can identify the tangent space $T_{p}(\mathcal{N})$ to $\mathcal{N}$ at a point $p$ with the subspace of the tangent space $T_{p}(M)$ consisting of vectors $v^{a}$ with $v^{a} \ell_{a}=0$. Since $\ell^{a} \equiv g^{a b} \ell_{b}$ lies in this subspace we can identify it with a vector field $\ell^{i}$ on $\mathcal{N}$, the integral curves of which are the null generators of the null surface. (Recall that we use lowercase Roman indices $i, j, \ldots$ to denote tensors intrinsic to $\mathcal{N}$.) Next, the pullback map takes covectors $w_{a}$ on $M$ evaluated on $\mathcal{N}$ to covectors $w_{i}$ on $\mathcal{N}$. We denote this pullback map by

$$
w_{a} \rightarrow \Pi_{i}^{a} w_{a}
$$

thereby defining the quantity $\Pi_{i}^{a}$. The pullback of the null normal covector $\ell_{a}$ vanishes identically by definition, since all vectors on $\mathcal{N}$ are orthogonal to $\ell_{a}$ :

$$
\Pi_{i}^{a} \ell_{a}=0 .
$$

A question that often arises in computations is when can a contraction $w_{a} v^{a}$ of spacetime tensors be replaced by a corresponding contraction $w_{i} v^{i}$ of tensors intrinsic to $\mathcal{N}$. First, given $w_{a}$ and $v^{a}$, while $w_{i}$ can be defined using the pullback, the quantity $v^{i}$ is not necessarily well defined; it is defined only when $\ell_{a} v^{a}=0$. When this condition is satisfied, the contractions coincide:

$$
\ell_{a} v^{a}=0 \quad \Longrightarrow \quad w_{a} v^{a}=w_{i} v^{i}
$$

A similar issue arises in going from three dimensions down to two dimensions. We denote by $W_{p}$ the two dimensional subspace of the dual space $T_{p}(\mathcal{N})^{*}$ consisting of covectors $w_{i}$ that satisfy $w_{i} \ell^{i}=0$. We will denote by abstract indices $A, B$ etc. tensors built on $W_{p}$. When can a contraction $w_{i} v^{i}$ of tensors on $\mathcal{N}$ be replaced by a corresponding contraction $w_{A} v^{A}$ of tensors in $W_{p}$ and $W_{p}^{*}$ ? The answer in this case is the opposite of that for going from four to three dimensions. First, given $w_{i}$ and $v^{i}$, the quantity $v^{A}$ is always well defined by considering $v^{i}$ as a linear map on $T_{p}(\mathcal{N})^{*}$ and restricting its action to $W_{p}$ (we shall call this operation a pullback). On the other hand, it is necessary that $w_{i} \ell^{i}=0$ in order that $w_{A}$ be defined. When this condition is satisfied, the contractions coincide:

$$
w_{i} \ell^{i}=0 \quad \Longrightarrow \quad w_{i} v^{i}=w_{A} v^{A} .
$$

\subsection{Geometric fields defined on a null hypersurface}

We denote by $q_{i j}$ the induced metric on $\mathcal{N}$

$$
q_{i j}=\Pi_{i}^{a} \Pi_{j}^{b} g_{a b},
$$

which has signature $(0,+,+)$. Taking the pullback of the relation $\ell_{a}=g_{a b} \ell^{b}$ and using eq. (3.7) gives

$$
q_{i j} \ell^{j}=0
$$

i.e., $\ell^{i}$ is a eigenvector of the induced metric with eigenvalue zero. It follows that we can regard $q_{i j}$ as a tensor in $W_{p} \otimes W_{p}$, which we write as $q_{A B}$. This has a unique inverse in $W_{p}^{*} \otimes W_{p}^{*}$ which we write as $q^{A B}$. We will use $q_{A B}$ and $q^{A B}$ to freely raise and lower capital Roman indices. 
The second fundamental form of the surface $\mathcal{N}$ is given by

$$
K_{i j}=\Pi_{i}^{a} \Pi_{j}^{b} \nabla_{a} \ell_{b}
$$

Since $\ell_{a}$ is normal to a hypersurface we have $\ell_{[a} \nabla_{b} \ell_{c]} \widehat{=} 0$ or $\nabla_{[a} \ell_{b]} \widehat{=} \ell_{[a} w_{b]}$ for some $w_{b}$, and taking the pullback and using (3.6) gives

$$
K_{[i j]}=0 .
$$

Similarly, lowering the index in eq. (3.2), taking the pullback and using eqs. (3.6) and (3.7) gives

$$
\ell^{i} K_{i j}=0 .
$$

It follows that $K_{i j}$ lies in $W_{p} \otimes W_{p}$ and so can be written as $K_{A B}$. We can uniquely decompose the second fundamental form as

$$
K_{A B}=\frac{1}{2} \theta q_{A B}+\sigma_{A B},
$$

where $\theta$ is the expansion and the shear $\sigma_{A B}$ is traceless, $q^{A B} \sigma_{A B}=0$. This equation can also be written as $K_{i j}=\theta q_{i j} / 2+\sigma_{i j}$.

The second fundamental form is related to the Lie derivative of the induced metric. Taking the pullback of the identity $£_{\ell} g_{a b}=2 \nabla_{(a} \ell_{b)}$ and using the fact that the pullback commutes with the Lie derivative gives

$$
K_{i j}=\frac{1}{2} £_{\ell} q_{i j} .
$$

Consider next the object

$$
\Pi_{i}^{a} \nabla_{a} \ell^{b}
$$

This tensor is orthogonal to the normal on the $b$ index, since $\ell_{b} \Pi_{i}^{a} \nabla_{a} \ell^{b}=\Pi_{i}^{a} \nabla_{a}\left(\ell_{b} \ell^{b}\right) / 2=0$, since $\ell_{b} \ell^{b}=0$ on $\mathcal{N}$ and the derivative is along the surface. Therefore this quantity is an intrinsic tensor which we write as

$$
\mathcal{K}_{i}^{j}
$$

called the Weingarten map [58]. From eqs. (3.2) and (3.7) it follows that

$$
\mathcal{K}_{i}^{j} \ell^{i}=\kappa \ell^{j}
$$

Similarly taking the pullback of the relation $\nabla_{a} \ell^{b} g_{b c}=\nabla_{a} \ell_{c}$ and using (3.7) and (3.9) gives that

$$
\mathcal{K}_{i}^{j} q_{j k}=K_{i k} .
$$

It follows from eqs. (3.12), (3.13), (3.18) and (3.19) that the Weingarten map $\mathcal{K}_{i}{ }^{j}$ has six independent nonzero components in general in four spacetime dimensions, three of which are determined by the second fundamental form $K_{i j}$, and one of which is determined by the non-affinity $\kappa$, leaving two additional independent components [see appendix A for more details, especially eqs. (A.13c) and (A.13d)]. 
Next, a choice of volume form $\varepsilon_{a b c d}$ on spacetime determines a volume form $\varepsilon_{i j k}$ on $\mathcal{N}$ as follows. We consider three-forms $\bar{\varepsilon}_{a b c}$ on $\mathcal{N}$ which satisfy

$$
4 \bar{\varepsilon}_{[a b c} \ell_{d]} \widehat{=} \varepsilon_{a b c d},
$$

and then take the pullback of these three-forms:

$$
\varepsilon_{i j k}=\Pi_{i}^{a} \Pi_{j}^{b} \Pi_{k}^{c} \bar{\varepsilon}_{a b c} .
$$

Although $\bar{\varepsilon}_{a b c}$ is not unique, its pullback $\varepsilon_{i j k}$ is. We define the antisymmetric tensor $\varepsilon^{i j k}$ by

$$
\varepsilon^{i j k} \varepsilon_{i j k}=3 !,
$$

and the two-form $\varepsilon_{i j}$ by

$$
\varepsilon_{i j}=-\varepsilon_{i j k} \ell^{k} .
$$

Under the scaling transformation (3.1) the various quantities defined in this subsection transform as

$$
\begin{aligned}
q_{i j} & \rightarrow q_{i j}, \\
K_{i j} & \rightarrow e^{\sigma} K_{i j}, \\
\mathcal{K}_{i}^{j} & \rightarrow e^{\sigma}\left(\mathcal{K}_{i}^{j}+D_{i} \sigma \ell^{j}\right), \\
\theta & \rightarrow e^{\sigma} \theta \\
\varepsilon_{i j k} & \rightarrow e^{-\sigma} \varepsilon_{i j k}, \\
\varepsilon^{i j k} & \rightarrow e^{\sigma} \varepsilon^{i j k} \\
\varepsilon_{i j} & \rightarrow \varepsilon_{i j}
\end{aligned}
$$

where $D_{i}$ is any derivative operator on $\mathcal{N}$.

\subsection{Divergence operator}

Although there is no preferred derivative operator on $\mathcal{N}$, one can define a divergence operation $v^{i} \rightarrow \hat{D}_{i} v^{i}$ on vector fields via

$$
\hat{D}_{i} v^{i}=\frac{1}{2} \varepsilon^{i j k} D_{k}\left(\varepsilon_{i j m} v^{m}\right),
$$

where $D_{i}$ is again any derivative operator on $\mathcal{N}$. The right hand side is independent of the choice of $D_{i}$ since it enters as an exterior derivative.

We can relate this divergence operator to the four dimensional divergence operator as follows. A vector field $v^{i}$ on $\mathcal{N}$ corresponds to a unique vector field $v^{a}$ on $\mathcal{N}$ with $v^{a} \ell_{a} \widehat{=} 0$. Now choose an extension of $v^{a}$ to a neighborhood of $\mathcal{N}$ in $M$. The linearized diffeomorphism associated with $v^{a}$ maps $\mathcal{N}$ into itself, and therefore preserves the normal $\ell_{a}$ up to a rescaling. Therefore there exists a function $\varpi$ on $\mathcal{N}$ which depends on $v^{a}$ such that

$$
£_{v} \ell_{a} \widehat{=} \varpi \ell_{a} .
$$


The relation between the two divergence operators is ${ }^{14}$

$$
\nabla_{a} v^{a} \widehat{=} \hat{D}_{i} v^{i}+\varpi
$$

The divergence of the normal is

$$
\hat{D}_{i} \ell^{i}=\theta
$$

This follows from the relation (3.27), the definition (3.26) of $\varpi$, and the trace of eq. (A.12).

\subsection{Stationary regions of null hypersurfaces}

As discussed in section 2.6 above, we shall call a region of a null surface stationary if there is a choice of normal covector $\tau_{a}$ in that region which satisfies Killings equation on the surface and to first order in deviations off the surface,

$$
\begin{aligned}
£_{\tau} g_{a b} & \widehat{=} 0, \\
\nabla_{c} £_{\tau} g_{a b} & \widehat{=} 0 .
\end{aligned}
$$

We will denote the corresponding value of $\kappa$ by $\kappa_{\tau}$, the surface gravity. Taking the pullback of eq. (3.29a) and using the fact that the pullback commutes with the Lie derivative gives

$$
£_{\tau} q_{i j}=0,
$$

and it follows from eq. (3.15) that

$$
K_{i j}=0,
$$

i.e. that the surface is shear free and expansion free.

It follows from the condition (3.31) together with eqs. (A.13) that the rotation one-form defined by

$$
\omega_{i}=-\mathcal{K}_{i}^{j} n_{j}
$$

where $n_{i}$ is any covector with $n_{i} \ell^{i}=-1$, is independent of the choice of $n_{i}$. This is true only for null surfaces that satisfy (3.31). Under the transformation (3.1) $\omega_{i}$ transforms as $\omega_{i} \rightarrow \omega_{i}+D_{i} \sigma$, from eqs. (3.24c) and (A.13).

We define

$$
\omega_{\tau i}=\left.\omega_{i}\right|_{\vec{\ell}=\vec{\tau}}
$$

to be the rotation one-form $\omega_{i}$ specialized to the choice of representative $\ell^{i}=\tau^{i} .{ }^{15}$ Now eq. (3.29) together with eq. (C.3.6) of Wald [50] imply that $£_{\tau} \nabla_{a} \tau^{b} \widehat{=} 0$, and taking a

\footnotetext{
${ }^{14}$ This relation can be derived by specializing to a coordinate system $\left(r, y^{1}, y^{2}, y^{3}\right)=\left(r, y^{\Gamma}\right)$ for which the hypersurface $\mathcal{N}$ is given by $r=0$ and with $\ell_{a} \widehat{=}(d r)_{a}$. Writing the volume form as $\varepsilon=e^{\Upsilon} d r \wedge d y^{1} \wedge d y^{2} \wedge d y^{3}$ for some function $\Upsilon$, the left hand side of eq. (3.27) can be written as

$$
e^{-\Upsilon} \partial_{r}\left(e^{\Upsilon} v^{r}\right)+e^{-\Upsilon} \partial_{\Gamma}\left(e^{\Upsilon} v^{\Gamma}\right)=\partial_{r} v^{r}+e^{-\Upsilon} \partial_{\Gamma}\left(e^{\Upsilon} v^{\Gamma}\right)
$$

The first term on the right hand side here is $\varpi$, while the second term is the intrinsic divergence $\hat{D}_{i} v^{i}$, by eqs. (3.20), (3.21) and (3.25).

${ }^{15}$ See Ashtekar [57] for an alternative method of defining $\omega_{\tau i}$.
} 
pullback yields $£_{\tau} \mathcal{K}_{i}{ }^{j}=0$. Combining this with eqs. (A.10) and (A.13) now shows that the nonaffinity and rotation one-form are Lie transported along the null surface:

$$
£_{\tau} \kappa_{\tau}=0, \quad £_{\tau} \omega_{\tau i}=0 .
$$

More generally, the Bardeen-Carter-Hawking derivation [59] of the zeroth law of black hole thermodynamics,

$$
D_{i} \kappa_{\tau}=0
$$

applies in this context, assuming the Einstein equations and the dominant energy condition. In the remainder of the paper we will be working in the context of vacuum general relativity, for which (3.35) will be satisfied in stationary regions.

\subsection{Orthonormal basis formalism}

Finally, it is sometimes useful for computational purposes to choose an auxiliary null vector field $n^{a}$ on $\mathcal{N}$ which together with $\ell^{a}$ forms part of an orthonormal basis. Some aspects of the formalism described above simplify when described in the language of an orthonormal basis, although that language does carry the baggage of an arbitrary choice. While the main results of this paper will not require a choice of auxiliary null vector, we will translate our results into the language of the orthonormal basis formalism since it is widely used. Details of the relation between the covariant and orthonormal basis formalisms for null surfaces are given in appendix A.

\section{Universal intrinsic structure of a null hypersurface}

In this section we will describe an intrinsic geometric structure on null hypersurfaces $\mathcal{N}$ that is determined by the spacetime geometry. It is universal in the sense that for a given $\mathcal{N}$ any two such structures are diffeomorphic. We will define the structure in section 4.1, and in section 4.2 we will describe the symmetry group of diffeomorphisms from $\mathcal{N}$ to $\mathcal{N}$ that preserve the structure. The corresponding Lie algebra is described in section 4.3; we will show in section 5 that this symmetry algebra coincides with that obtained from a particular definition of covariant phase space for general relativity with a null boundary in the Wald-Zoupas approach. Section 4.4 discusses preferred subalgebras associated with stationary regions of the null hypersurface. Finally in section 4.5 we discuss how the group and algebra are modified in the case where the null hypersurface has a boundary $\partial \mathcal{N}$ in $M$.

\subsection{Definition of intrinsic structure}

Consider a manifold $\mathcal{N}$ which is equipped with a smooth, nowhere vanishing vector field $\ell^{i}$ and a smooth function $\kappa$. Letting $\mathcal{Z}$ denote the manifold of integral curves, we assume that $\mathcal{N}$ is diffeomorphic to the product $\mathcal{Z} \times \mathbb{R}$. We define an equivalence relation on such pairs $\left(\ell^{i}, \kappa\right)$ by saying that two pairs are equivalent if they are related by a rescaling of the form [cf. eqs. (3.1) and (3.3) above]

$$
\begin{aligned}
\ell^{i} & \rightarrow e^{\sigma} \ell^{i}, \\
\kappa & \rightarrow e^{\sigma}\left(\kappa+£_{\ell} \sigma\right)
\end{aligned}
$$


where $\sigma$ is a smooth function on $\mathcal{N}$. We denote by

$$
\mathfrak{u}=\left[\ell^{i}, \kappa\right]
$$

the equivalence class associated with $\left(\ell^{i}, \kappa\right)$. A choice of equivalence class is the desired intrinsic geometric structure on $\mathcal{N}$.

Suppose now we are given a spacetime $\left(M, g_{a b}\right)$ with null boundary $\mathcal{N}$. The spacetime geometry then determines a structure $\left[\ell^{i}, \kappa\right]$ in the manner described in section 3 above: the vector $\ell^{i}$ is obtained by raising the index on a choice of normal covector, and $\kappa$ is the non-affinity of that vector. The resulting equivalence class $\left[\ell^{i}, \kappa\right]$ is independent of the choice of normalization of the covector, by the equivalence relation (4.1).

The intrinsic structure determines a class of foliations of $\mathcal{N}$ as follows. Choose a cross section $\mathcal{S}$ of $\mathcal{N}$, a surface which each integral curve intersects exactly once, which will be diffeomorphic to the base space $\mathcal{Z}$. Out of the equivalence class $\left[\ell^{i}, \kappa\right]$, pick a member $\left(\ell_{0}^{i}, 0\right)$ for which the non-affinity vanishes, by starting with a general member $\left(\ell^{i}, \kappa\right)$ and solving the differential equation $\kappa+£_{\ell} \sigma=0$ for the scaling function $\sigma$. Now Lie drag the cross section $\mathcal{S}$ along integral curves of $\ell_{0}^{i}$. The resulting foliation ${ }^{16}$ will be level sets of a coordinate $u$ which is determined by the properties that $u=0$ on $\mathcal{S}$ and $\ell_{0}^{i} D_{i} u=1$. In addition, if $\theta^{A}$ is any coordinate system on $\mathcal{S}$, one can extend the definition of these coordinates to $\mathcal{N}$ by demanding that they be constant along the integral curves, thereby generating a coordinate system $\left(u, \theta^{A}\right)$ on $\mathcal{N}$ for which $\vec{\ell}_{0}=\partial_{u}$.

We will say that an intrinsic structure is complete if all of the generators of $\mathcal{N}$ can be extended to arbitrary values of affine parameter in both directions (where $u$ is an affine parameter if $\vec{\ell}=\partial_{u}$ with $\kappa=0$ ). For example, the future light cone of a point $\mathcal{P}$ in Minkowski spacetime (with $\mathcal{P}$ itself removed) is not complete when the intrinsic structure induced by the flat Minkowski metric is used, since all of the generators start at $\mathcal{P}$. By contrast, the event horizon in maximally extended Schwarzschild is complete (see appendix E). We will study both types of intrinsic structure later in this paper.

Given two different complete intrinsic structures $\mathfrak{u}=\left[\ell^{i}, \kappa\right]$ and $\mathfrak{u}^{\prime}=\left[\ell^{\prime i}, \kappa^{\prime}\right]$ on $\mathcal{N}$, there exists a diffeomorphism $\varphi: \mathcal{N} \rightarrow \mathcal{N}$ which maps $\mathfrak{u}$ onto $\mathfrak{u}^{\prime}$. In this sense the complete intrinsic structure is universal, in the same way that an intrinsic structure of a different kind on future null infinity is universal in the BMS construction [60]. The existence of the diffeomorphism $\varphi$ can be shown as follows. Choose a cross section $\mathcal{S}$ of $\mathcal{N}$, and using $\mathfrak{u}$ construct a coordinate $u$ on $\mathcal{N}$ in the manner discussed above. Define the diffeomorphism

$$
\Phi=(u, \pi): \mathcal{N} \rightarrow \mathbb{R} \times \mathcal{Z}
$$

where $\pi: \mathcal{N} \rightarrow \mathcal{Z}$ is the natural projection obtained by taking each point to the corresponding integral curve. Starting from the intrinsic structure $\mathfrak{u}^{\prime}$ one can similarly define a diffeomorphism $\Phi^{\prime}$, and then $\varphi=\Phi^{-1} \circ \Phi^{\prime}$ maps $\mathfrak{u}$ onto $\mathfrak{u}^{\prime}$.

\footnotetext{
${ }^{16}$ The class of foliations generated in this way has considerable freedom. One can pick the initial cross section $\mathcal{S}$ arbitrarily, and in addition one can pick a second arbitrary cross section $\mathcal{S}^{\prime}$ disjoint from $\mathcal{S}$ and arrange for it to belong to the foliation, by exploiting the rescaling freedom $\ell_{0}^{i} \rightarrow e^{\sigma} \ell_{0}^{i}$ with $£_{\ell_{0}} \sigma=0$. However, once $\mathcal{S}$ and $\mathcal{S}^{\prime}$ are specified, the foliation is uniquely determined.
} 


\subsection{Symmetry group of a complete intrinsic structure}

We now turn to a discussion of the symmetry group $G_{\mathfrak{u}}$ of diffeomorphisms $\varphi: \mathcal{N} \rightarrow \mathcal{N}$ which preserve a universal structure $\mathfrak{u}$. Remarkably, the structure of this group is very similar to that of the BMS group at null infinity, but with two important differences. First, the Lorentz group at null infinity is replaced by the group $\operatorname{Diff}(\mathcal{Z})$ of diffeomorphisms of the base space $\mathcal{Z}$, typically the two-sphere $S^{2}$. This replacement is not surprising, since the conformal freedom that is used at null infinity to map the induced metric onto a metric of constant curvature is not present for general null surfaces. Second, the abelian subgroup of supertranslations at null infinity is replaced by a nonabelian subgroup, which contains angle-dependent displacements of affine parameters and rescalings of affine parameters.

From the definition (4.1) of the equivalence class, it follows that a diffeomorphism $\varphi: \mathcal{N} \rightarrow \mathcal{N}$ is a symmetry in $G_{\mathfrak{u}}$ if, for a given representative $\left(\ell^{i}, \kappa\right)$ in $\mathfrak{u}$, the pullback $\varphi_{*}$ acts as a scaling transformation for some smooth scaling function $\beta=\beta(\varphi)$ on $\mathcal{N}$ [cf. eqs. (4.1) above]:

$$
\begin{aligned}
\varphi_{*} \ell^{i} & =e^{\beta} \ell^{i}, \\
\varphi_{*} \kappa & =e^{\beta}\left(\kappa+£_{\ell} \beta\right) .
\end{aligned}
$$

If we choose a different representative $\left(\ell^{\prime i}, \kappa^{\prime}\right)$ with $\ell^{\prime i}=e^{\sigma} \ell^{i}$, then we find from (4.4) that

$$
\begin{aligned}
& \varphi_{*} \ell^{\prime i}=e^{\beta^{\prime}} \ell^{i}, \\
& \varphi_{*} \kappa^{\prime}=e^{\beta^{\prime}}\left(\kappa^{\prime}+£_{\ell^{\prime}} \beta^{\prime}\right),
\end{aligned}
$$

where

$$
\beta^{\prime}=\beta+\varphi_{*} \sigma-\sigma .
$$

Hence $\varphi$ will be a symmetry if (4.4) is satisfied for any choice of representative.

Specialize now to a choice of coordinate system $\left(u, \theta^{A}\right)$ and representative of the kind discussed in section 4.1 above, where $\kappa=0$ and $\vec{\ell}=\partial_{u}$. Then the general solution for a diffeomorphism that satisfies (4.4) is $\left(u, \theta^{A}\right) \rightarrow\left(\bar{u}, \bar{\theta}^{A}\right)$, where

$$
\begin{aligned}
\bar{u}\left(u, \theta^{A}\right) & =\alpha\left(\theta^{A}\right)+e^{-\beta\left(\theta^{A}\right)} u \\
\bar{\theta}^{A}\left(u, \theta^{B}\right) & =\bar{\theta}^{A}\left(\theta^{B}\right) .
\end{aligned}
$$

This group of transformations contains a number different subgroups:

- The subgroup with $\alpha=0, \beta=0$, which consists of arbitrary diffeomorphisms on the base space $\mathcal{Z}, \operatorname{Diff}(\mathcal{Z})$. In many applications this will be $\operatorname{Diff}\left(S^{2}\right)$, the diffeomorphisms of the two-sphere. These transformations have also been called superrotations [15].

- The subgroup with $\bar{\theta}^{A}=\theta^{A}$, parameterized by $\alpha\left(\theta^{A}\right)$ and $\beta\left(\theta^{A}\right)$. These transformations consist of reparameterizations of the generators of the null surface. ${ }^{17}$ We will call

\footnotetext{
${ }^{17}$ This supertranslation subgroup of symmetries played an important role in Wall's proof of the generalized second law [27].
} 
these transformations supertranslations, following common use $[10,12,13,15,16,18$, 21, 61-63], and because of the analogy with the supertranslations of the BMS group.

- The subgroup of the supertranslation group with $\beta=0, \bar{\theta}^{A}=\theta^{A}$, which is parameterized by $\alpha\left(\theta^{A}\right)$. We will call these transformations affine supertranslations since they consist of angle-dependent displacements in affine parameter (as opposed to angle-dependent displacements in Killing parameter or Killing supertranslations [10, $12,13,15,21,61-63]$, to be discussed in section 4.4 below.)

- The subgroup of the supertranslation group with $\alpha=0, \bar{\theta}^{A}=\theta^{A}$, which is parameterized by $\beta\left(\theta^{A}\right)$. These transformations consist of constant rescalings of affine parameter on each generator. (Note however that if $\tau=\ln (u) / \kappa$ is a Killing parameter, the transformations consist of angle-dependent displacements in $\tau$; see section 4.4.)

The first subgroup preserves the foliation associated with the coordinate system $\left(u, \theta^{A}\right)$, while the last three preserve the integral curves. The affine supertranslation and supertranslation subgroups do not depend on the choice of coordinate system or representative, and can be invariantly defined. The rescaling and $\operatorname{Diff}(\mathcal{Z})$ subgroups, by contrast, do depend on these choices. Their status is analogous to that of Lorentz subgroups of the BMS group: there are many such subgroups, but no natural or unique choice.

The symmetry algebra associated with the group of transformations is given by the linearization of eq. (4.7), which yields the vector field

$$
\vec{\chi}=\left[\alpha\left(\theta^{A}\right)-\beta\left(\theta^{A}\right) u\right] \partial_{u}+X^{A}\left(\theta^{B}\right) \partial_{A},
$$

where $X^{A}$ is arbitrary. The algebra of these generators under Lie brackets is

$$
\left[\left(\alpha_{1}-\beta_{1} u\right) \partial_{u}+X_{1}^{A} \partial_{A},\left(\alpha_{2}-\beta_{2} u\right) \partial_{u}+X_{2}^{A} \partial_{A}\right]=\left(\alpha_{3}-\beta_{3} u\right) \partial_{u}+X_{3}^{A} \partial_{A}
$$

with

$$
\begin{aligned}
\alpha_{3} & =-\alpha_{1} \beta_{2}+X_{1}^{A} \partial_{A} \alpha_{2}+\alpha_{2} \beta_{1}-X_{2}^{A} \partial_{A} \alpha_{1}, \\
\beta_{3} & =-X_{1}^{A} \partial_{A} \beta_{2}+X_{2}^{A} \partial_{A} \beta_{1} \\
X_{3}^{A} & =X_{1}^{B} \partial_{B} X_{2}^{A}-X_{2}^{B} \partial_{B} X_{1}^{A} .
\end{aligned}
$$

While these explicit coordinate expressions are convenient, it can be difficult to discern which aspects of the structures are specific to the choice of coordinate system. We now turn to an analysis of the symmetry algebra which is covariant and does not depend on a choice of coordinates.

\subsection{Symmetry algebra of a complete intrinsic structure}

The Lie algebra $\mathfrak{g}_{\mathfrak{u}}$ of infinitesimal symmetries in $G_{\mathfrak{u}}$ consists of vector fields $\chi^{i}$ on $\mathcal{N}$ which obey the linearized versions of eqs. (4.4):

$$
\begin{aligned}
& £_{\chi} \ell^{i}=\beta \ell^{i}, \\
& £_{\chi} \kappa=\beta \kappa+£_{\ell} \beta .
\end{aligned}
$$


As before, if these equations are satisfied for one representative $\left(\ell^{i}, \kappa\right)$ of the equivalence class, they will be satisfied for all representatives. The function $\beta$ depends on both the symmetry $\chi^{i}$ and on the representative $\ell^{i}, \beta=\beta\left(\chi^{i}, \ell^{i}\right)$, and the dependence on the normalization is given by the linearized version of eq. (4.6):

$$
\beta\left(\chi^{i}, e^{\sigma} \ell^{i}\right)=\beta\left(\chi^{i}, \ell^{i}\right)+£_{\chi}^{\sigma} .
$$

The general solution of eqs. (4.11) for $\chi^{i}$, with a choice of representative and coordinate system $\left(u, \theta^{A}\right)$ for which $\kappa=0$ and $\vec{\ell}=\partial_{u}$, is given by eq. (4.8) above.

The algebra $\mathfrak{g}_{\mathfrak{u}}$ inherits the Lie bracket structure of the space of vector fields on $\mathcal{N}$. From the definition of the symmetry group $G_{\mathfrak{u}}$ as a subgroup of $\operatorname{Diff}(\mathcal{N})$, it follows that $\mathfrak{g}_{\mathfrak{u}}$ is closed under this Lie bracket. This closure was also shown in eq. (4.10) above, and can also be checked directly in the covariant context: if $\vec{\chi}_{1}$ and $\vec{\chi}_{2}$ are two vector fields which satisfy eqs. (4.11), then $\vec{\chi}_{3}=\left[\vec{\chi}_{1}, \vec{\chi}_{2}\right]$ also satisfies eqs. (4.11) with

$$
\beta\left(\vec{\chi}_{3}\right)=£_{\chi_{1}} \beta_{2}-£_{\chi_{2}} \beta_{1},
$$

where $\beta_{1}=\beta\left(\vec{\chi}_{1}\right)$ and $\beta_{2}=\beta\left(\vec{\chi}_{2}\right)$.

We now argue that the symmetry algebra has the structure

$$
\mathfrak{g}_{\mathfrak{u}} \cong \operatorname{diff}(\mathcal{Z}) \ltimes\left(\mathfrak{b} \ltimes \mathfrak{s}_{0}\right),
$$

where $\ltimes$ denotes semidirect sum and the various algebras are as follows:

- $\operatorname{diff}(\mathcal{Z})$ is the algebra of linearized diffeomorphisms of the base space $\mathcal{Z}$, i.e., vector fields on $\mathcal{Z}$.

- $\mathfrak{s}_{0}$ is the abelian algebra of linearized affine supertranslations, consisting of vector fields of the form

$$
\chi^{i}=f \ell^{i}
$$

where the function $f$ on $\mathcal{N}$ satisfies

$$
£_{\ell} f+\kappa f=0 .
$$

- $\mathfrak{b}$ is an abelian algebra of linearized rescalings such that $\mathfrak{b} \ltimes \mathfrak{s}_{0} \cong \mathfrak{s}$, where $\mathfrak{s}$ is the algebra of linearized supertranslations. This is the algebra consisting of vector fields of the form (4.15) where the function $f$ satisfies

$$
£_{\ell}\left(£_{\ell} f+\kappa f\right)=0 .
$$

We now turn to the derivation of the structure (4.14). We define the subspace

$$
\mathfrak{s}=\left\{\chi^{i} \in \mathfrak{g}_{\mathfrak{u}} \mid \chi^{i}=f \ell^{i} \text { for some } f\right\} .
$$

By comparison with eqs. (4.7) and (4.8), we see that this subspace consists of the linearized supertranslations. Inserting the definition (4.18) into eqs. (4.11) yields that the function $f$ satisfies the condition (4.17) with

$$
\beta(f \vec{\ell})=-£_{\ell} f .
$$


The condition (4.17) is invariant under the scaling transformations

$$
\vec{\ell} \rightarrow e^{\sigma} \vec{\ell}, \quad f \rightarrow e^{-\sigma} f
$$

so the subspace $\mathfrak{s}$ is parameterized by functions of scaling weight 1 on $\mathcal{N}$ [cf. eq. (3.4)]. The subspace $\mathfrak{s}$ is closed under the Lie bracket and so is a subalgebra; we have

$$
\left[f_{1} \vec{\ell}, f_{2} \vec{\ell}\right]=\left(f_{1} £_{\ell} f_{2}-f_{2} £_{\ell} f_{2}\right) \vec{\ell}
$$

Since the right hand side is nonvanishing in general, the subalgebra is nonabelian. Finally, for any $f \vec{\ell} \in \mathfrak{s}$ and any $\vec{\chi} \in \mathfrak{g}_{\mathfrak{u}}$, we have from eqs. (4.11) that

$$
[f \vec{\ell}, \vec{\chi}]=-\left[£_{\chi} f+\beta(\vec{\chi}) f\right] \vec{\ell} .
$$

Hence $\left[\mathfrak{s}, \mathfrak{g}_{\mathfrak{u}}\right] \subseteq \mathfrak{s}$, so $\mathfrak{s}$ is a Lie ideal of $\mathfrak{g}_{\mathfrak{u}}$.

Next, we define the subalgebra $\mathfrak{s}_{0}$ of $\mathfrak{s}$ by

$$
\mathfrak{s}_{0}=\left\{f \ell^{i} \mid £_{\ell} f+\kappa f=0\right\} .
$$

By comparison with eqs. (4.7) and (4.8), we see that this subalgebra consists of the linearized affine supertranslations, and it follows from eq. (4.21) that it is abelian. The definition (4.23) is invariant under the rescalings (4.20). If we choose a representative $\left(\ell^{i}, \kappa\right)$ of the equivalence class with $\kappa=0$, it follows that $f$ is constant along generators and so can be regarded as a function on $\mathcal{Z}$. There is a residual rescaling freedom of the form (4.20) with $£_{\ell} \sigma=0$ that preserves $\kappa=0$. Hence, the algebra $\mathfrak{s}_{0}$ can be identified with functions on the base space $\mathcal{Z}$ of scaling weight 1 , from eq. (3.4), just like supertranslations on $\mathscr{I}{ }^{18}$

Next, if $f_{1} \vec{\ell}$ and $f_{2} \vec{\ell}$ are elements of $\mathfrak{s}$, it follows from eq. (4.17) that

$$
\left(£_{\ell}+\kappa\right)\left(f_{1} £_{\ell} f_{2}-f_{2} £_{\ell} f_{2}\right)=0 .
$$

Combining this with eq. (4.21) shows that

$$
[\mathfrak{s}, \mathfrak{s}] \subseteq \mathfrak{s}_{0}
$$

so $\mathfrak{s}_{0}$ is a Lie ideal of $\mathfrak{s}$. We define the quotient algebra $\mathfrak{b} \cong \mathfrak{s} / \mathfrak{s}_{0}$. This consists of equivalence classes of elements of $\mathfrak{s}$, where $f_{1} \vec{\ell} \sim f_{2} \vec{\ell}$ if $£_{\ell} f_{1}+\kappa f_{1}=£_{\ell} f_{2}+\kappa f_{2}$. Elements of $\mathfrak{b}$ can be parameterized in terms of functions ${ }^{19}$ on $\mathcal{Z}$ of scaling weight zero, and they correspond to linearized rescalings, cf. eq. (4.8) above. It follows from eq. (4.25) that $\mathfrak{b}$ is abelian, and so we obtain

$$
\mathfrak{s} \cong \mathfrak{b} \ltimes \mathfrak{s}_{0}
$$

where both $\mathfrak{b}$ and $\mathfrak{s}_{0}$ are abelian.

\footnotetext{
${ }^{18}$ Unlike the case with the BMS algebra, there is no preferred translation subalgebra of the affine supertranslation algebra $\mathfrak{s}_{0}$. Even if $\mathcal{Z}$ is topologically $S^{2}$, there is no universal metric on $\mathcal{Z}$, so it is not possible to single out a 4-dimensional subalgebra of translations by the first four spherical harmonics. Also, there is no scaling-invariant notion of constant functions on $\mathcal{Z}$, so there is not even a natural way to single out "time-translations".

${ }^{19}$ Essentially the functions $£_{\ell} f+\kappa f$ projected to $\mathcal{Z}$, where $f \vec{\ell} \in \mathfrak{s}$.
} 
We next argue that the quotient algebra $\mathfrak{g}_{\mathfrak{u}} / \mathfrak{s}$ is isomorphic to the algebra of linearized diffeomorphisms on the base space $\mathcal{Z}$,

$$
\mathfrak{g}_{\mathfrak{u}} / \mathfrak{s} \cong \operatorname{diff}(\mathcal{Z})
$$

which when combined with eq. (4.26) gives the algebra structure ${ }^{20}(4.14)$. The algebra $\mathfrak{g}_{\mathfrak{u}} / \mathfrak{s}$ consists of equivalence classes $\left[\chi^{i}\right]$ of vector fields $\chi^{i}$ in $g_{\mathfrak{u}}$, where two vector fields are equivalent if they differ by an element $f \ell^{i}$ in $\mathfrak{s}$. Pick a cross section $\mathcal{S}$ of $\mathcal{N}$, and denote by $n_{i}$ the unique normal covector to $\mathcal{S}$ whose normalization is fixed by $n_{i} \ell^{i}=-1$. Given an equivalence class $\left[\chi^{i}\right]$, one can find a member $\chi^{i}$ with $\chi^{i} n_{i}=0$ on $\mathcal{S}$, by using the freedom to add terms of the form $f \ell^{i}$ and using the fact that solutions to eq. (4.17) can be freely specified on an initial cross section $\mathcal{S}$. This member $\chi^{i}$ can then be regarded as a vector field $\chi^{A}$ on $\mathcal{S}$, and by using the natural identification of $\mathcal{S}$ and $\mathcal{Z}$, as a vector field on $\mathcal{Z}$. We have thus defined a mapping from $\mathfrak{g}_{\mathfrak{u}} / \mathfrak{s}$ to $\operatorname{diff}(\mathcal{Z})$. One can check that this mapping is onto, and it follows from eqs. (4.11) that the identification of $\mathfrak{g}_{\mathfrak{u}} / \mathfrak{s}$ and $\operatorname{diff}(\mathcal{Z})$ is independent of the choice of cross section $\mathcal{S}$. Thus we have derived the decomposition (4.14) of the algebra $\mathfrak{g}_{\mathfrak{u}}$.

For the computations of charges in section 6 below, it will be useful to use an explicit decomposition of symmetry generators $\vec{\chi}$ into different pieces. However, because of the semi-direct structure $\mathfrak{g} \cong \operatorname{diff}(\mathcal{Z}) \ltimes \mathfrak{s}$, there is no natural way to decompose a generator $\chi^{i}$ into a $\mathfrak{s}$-part and a $\operatorname{diff}(\mathcal{Z})$-part. Such a decomposition requires an arbitrary choice of origin in $\mathfrak{s}$. We make such a choice by choosing a smooth covector $n_{i}$ on $\mathcal{N}$, normalized so that

$$
n_{i} \ell^{i}=-1 .
$$

The generator $\chi^{i}$ can then be uniquely decomposed as

$$
\chi^{i}=f \ell^{i}+X^{i}
$$

where

$$
X^{i} n_{i}=0
$$

Here the first term $f \ell^{i}$ parameterizes the supertranslations, and the second term $X^{i}$ parameterizes the diffeomorphisms on the base space.

In order for both terms on the right hand side of eq. (4.29) to belong to $\mathfrak{g}_{\mathfrak{u}}$, from eq. (4.17) it is necessary that

$$
£_{\ell}\left(£_{\ell}+\kappa\right)\left(\chi^{i} n_{i}\right)=0 .
$$

Using eq. (4.11), this will be automatically satisfied if $n_{i}$ obeys the equation

$$
£_{\ell}\left(£_{\ell}+\kappa\right) n_{i}+D_{i} \kappa=0
$$

where $D_{i}$ is any derivative operator on $\mathcal{N}$. This equation is invariant under the rescalings (4.1), since $n_{i}$ transforms as $n_{i} \rightarrow e^{-\sigma} n_{i}$ from eq. (4.28). If we choose $n_{i}$ to be the normal covector to a foliation of surfaces in the natural class of foliations discussed in section 4.1 above, normalized according to (4.28), then the condition (4.32) is satisfied.

\footnotetext{
${ }^{20}$ The subalgebra $\mathfrak{s}_{0}$ is also a Lie ideal of $\mathfrak{g}_{\mathfrak{u}}$, but $\mathfrak{g} / \mathfrak{s}_{0} \neq \operatorname{diff}(\mathcal{Z}) \ltimes \mathfrak{b}$.
} 


\subsection{Preferred subalgebra for stationary regions of a null hypersurface: Killing supertranslations}

Stationary regions of the hypersurface $\mathcal{N}$ that intersect all the generators determine a preferred subalgebra $\mathfrak{t}$ of the supertranslation algebra $\mathfrak{s}$. This algebra is the set of vector fields $\chi^{i}$ in $\mathfrak{s}$ for which

$$
£_{\tau} \chi^{i}=0
$$

in the stationary region, where $\tau^{a}$ is the Killing vector field which is normal to $\mathcal{N}$. Since $\left(\tau^{i}, \kappa_{\tau}\right)$ is a representative of the equivalence class, we have from eq. (4.17) that all elements $\chi^{i}$ of $\mathfrak{s}$ satisfy

$$
£_{\tau}\left(£_{\tau}+\kappa_{\tau}\right) \chi^{i}=0 .
$$

Hence it follows from eqs. (3.34) and (4.33) that all solutions of eq. (4.33) in the stationary region can be extended to vector fields on all of $\mathcal{N}$ which lie in $\mathfrak{s}$.

To get some insight into the nature of this subalgebra, ${ }^{21}$ specialize to a representative $\left(\ell^{i}, \kappa\right)$ and a coordinate system $\left(u, \theta^{A}\right)$ where $\vec{\ell}=\partial_{u}$ and $\kappa=0$, where the general solution for $\chi^{i}$ is given by eq. (4.8). Then the Killing field $\tau^{i}$ will be of the form $\tau^{i}=\kappa_{\tau}\left(u-u_{0}\right) \ell^{i}$, by eqs. (3.3), (3.34) and (3.35), where $\kappa_{\tau}$ is a constant and $u_{0}$ is a function of $\theta^{A}$ but independent of $u$. The subalgebra $t$ is then given by the condition

$$
\alpha-\beta u_{0}=0,
$$

and consists of vector fields of the form $\chi^{i}=-\left(\beta / \kappa_{\tau}\right) \tau^{i}$. The corresponding transformation (4.7) can be expressed as

$$
\bar{\tau}=\tau-\frac{\beta}{\kappa_{\tau}},
$$

where we have defined a Killing parameter $\tau$ by $\vec{\tau}=d / d \tau$. We will call these angledependent displacements of Killing parameter Killing supertranslations. They have been studied in refs. [10, 12, 13, 15, 21, 61-63] (although they are often called just supertranslations). The intersection of the Killing supertranslation subalgebra $\mathfrak{t}$ with the affine supertranslation subalgebra $\mathfrak{s}_{0}$ will generically have dimension 0 .

We note that the Killing supertranslation subalgebra $\mathfrak{t}$ can be defined under the slightly weaker hypothesis that the region of $\mathcal{N}$ is weakly isolated in the sense of Ashtekar [57], which implies that it is shear and expansion free, satisfies eqs. (3.34), and possesses a preferred choice of normal up to constant rescalings.

\subsection{Symmetry groups of null hypersurfaces with boundaries}

Our analysis so far has been restricted by the assumptions that the null hypersurface $\mathcal{N}$ has topology $\mathcal{Z} \times \mathbb{R}$, and that the intrinsic structure is complete, that is, that the generators of the null surface extend to infinite affine parameters in both directions. We now discuss how the symmetry group is modified when these assumptions are relaxed. Specifically, we will consider incomplete intrinsic structures. These generally occur when the null hypersurface

\footnotetext{
${ }^{21}$ The pullback $\tau^{i}$ of the Killing field is itself a member of the subspace $\mathfrak{t}$, giving a preferred onedimensional subspace of "translations".
} 
$\mathcal{N}$ has a nontrivial topological boundary $\partial \mathcal{N}$ in $M \cdot{ }^{22}$ Rather than give a general analysis of the different possibilities, we will discuss two specific examples.

The first example is the future light cone of a point $\mathcal{P}$ in a spacetime which is spherically symmetric about $\mathcal{P}$. This could be the future event horizon of a black hole in a spherically symmetric gravitational collapse spacetime. Or, it could be the future light cone of a point in Minkowski spacetime. The null hypersurface still has topology $\mathcal{Z} \times \mathbb{R} \simeq S^{2} \times \mathbb{R}$ (if the point $\mathcal{P}$ is excluded), but has the nontrivial boundary $\partial \mathcal{N}=\{\mathcal{P}\}$. The induced intrinsic structure is incomplete if the metric is smooth in a neighborhood of $\mathcal{P}$, as all the generators start at $\mathcal{P}$.

The second example is the future event horizon in the maximally extended Schwarzschild spacetime, on one of the two branches. In this case the boundary of $\mathcal{N}$ is the bifurcation twosphere, and the induced intrinsic structure is again incomplete, as all the generators start on the bifurcation twosphere.

In these cases, the definition of the symmetry group is modified to include the requirement that it preserve the boundary:

$$
G_{\mathfrak{u}}=\left\{\varphi: \mathcal{N} \rightarrow \mathcal{N} \mid \varphi_{*} \mathfrak{u}=\mathfrak{u}, \quad \varphi(\partial \mathcal{N})=\partial \mathcal{N}\right\} .
$$

In the first case of a single point, $\partial \mathcal{N}=\{\mathcal{P}\}$, the corresponding Lie algebra consists of the vector fields $\chi^{i}$ which satisfy eqs. (4.11) and in addition the condition

$$
\left.\chi^{i}\right|_{\partial \mathcal{N}}=0 .
$$

This removes the affine supertranslations and but not the rescalings or $\operatorname{diff}\left(S^{2}\right) \operatorname{diffeo-}$ morphisms. If one chooses an affine coordinate system $\left(u, \theta^{A}\right)$ of the type described in section 4.1 , specialized so that $u=0$ on $\partial \mathcal{N}$, then the transformation group (4.7) is modified by the condition.

$$
\alpha=0 .
$$

In the second case of the bifurcation twosphere, the condition (4.38) is replaced by the requirement that the vector field be tangent to $\partial \mathcal{N}$ on $\partial \mathcal{N}$,

$$
\left.\chi^{i} n_{i}\right|_{\partial \mathcal{N}}=0,
$$

where $n_{i}$ is the normal to $\partial \mathcal{N}$. The modification to the algebra is the same as in the first case, given by the condition (4.39).

We note that in this context the Killing supertranslation subalgebra $\mathfrak{t}$ associated with stationary regions of the null surface will generically have dimension 0 , by eqs. (4.35) and (4.39). This is discussed further in section 7 below (footnote 28).

\section{General relativity with a null boundary: covariant phase space}

As discussed in section 2, the starting point of the Wald-Zoupas framework is the definition of a field configuration space $\mathscr{F}$ of kinematically allowed field configurations, and the

\footnotetext{
${ }^{22}$ The hypersurface $\mathcal{N}$ can have a nontrivial boundary only when $\mathcal{N}$ is a proper subset of the boundary $\partial M$ of $M$, as it will be in typical applications, since $\partial \partial M=\{\}$.
} 
corresponding covariant phase space $\overline{\mathscr{F}} \subset \mathscr{F}$ obtained by restricting attention to on-shell field configurations. In this section we give a particular version of these definitions for general relativity in the presence of a null boundary in $3+1$ dimensions. The definition is given in section 5.1, and in section 5.2 we show that the symmetry group and algebra associated with this field configuration space coincide with those of the universal intrinsic structure of the null surface discussed in section 4.

\subsection{Definition of field configuration space}

Consider a manifold $M$ with boundary, for which a manifold $\mathcal{N}$ is a portion of the boundary. We would like to consider the space $\mathscr{F}_{0}$ consisting of smooth metrics $g_{a b}$ on $M$ for which the boundary $\mathcal{N}$ is null and for which the induced boundary structure on $\mathcal{N}$ is complete. This space $\mathscr{F}_{0}$ is not the field configuration space $\mathscr{F}$ we seek, since it contains a considerable amount of diffeomorphism redundancy. We will obtain our definition of $\mathscr{F}$ by fixing some of this freedom.

The kinds of fixing of diffeomorphism freedom that we will allow will be restricted by three general considerations:

- They must be global on the field configuration space, not restricted to on-shell configurations.

- They must be local to the boundary in the sense that the diffeomorphisms needed to enforce the gauge condition can be computed from degrees of freedom on the boundary.

- Field configurations (metrics in this case) and their derivatives evaluated on the boundary induce on the boundary certain geometric structures, which can be divided into universal and non-universal structures. The universal structures are the same for all field configurations (up to boundary diffeomorphisms), while the nonuniversal ones depend on the field configuration. We restrict attention to fixings of the diffeomorphism freedom that involve only the universal structures.

The diffeomorphism (and conformal freedom) fixings used at future null infinity by Wald and Zoupas [6] are also of this type.

As a side note, as discussed in section 2.3 above, gauge in this context is not synonymous with diffeomorphism freedom, since there are some diffeomorphisms that act on the boundary which do not correspond to degeneracies of the symplectic form on phase space (a more fundamental notion of gauge). Some of the diffeomorphism freedom we fix in going from $\mathscr{F}_{0}$ to $\mathscr{F}$ is not gauge in this sense. For this reason, it would be desirable to consider a larger field configuration space that includes all metric variations that are not degeneracy directions of the symplectic form. In appendix $\mathrm{H}$ we explore a modification of our definition of the field configuration space which yields a modified and larger algebra of symmetries and a modified set of charges. The main drawback of this modification is that it is no longer possible to obtain uniqueness of the prescription for defining localized charges by demanding that fluxes vanishes for stationary solutions, as discussed in section 2.6 above. It is possible that a unique prescription may be obtained from some other criterion. 
Our definition of the field configuration space $\mathscr{F}$ proceeds as follows. We start by defining a particular geometric structure on $\mathcal{N}$ which we will call a boundary structure. We consider triples $\left(\ell^{a}, \kappa, \hat{\ell}_{a}\right)$ of fields on $\mathcal{N}$, where $\ell^{a}$ is a smooth, nowhere vanishing vector field, $\kappa$ is a smooth function, $\hat{\ell}_{a}$ is a choice of normal covector ${ }^{23}$ to $\mathcal{N}$, and

$$
\ell^{a} \hat{\ell}_{a} \widehat{=} 0
$$

Recall that we are using $\widehat{=}$ to mean equality when restricted to $\mathcal{N}$. We define two such triples $\left(\ell^{a}, \kappa, \hat{\ell}_{a}\right)$ and $\left(\ell^{\prime a}, \kappa^{\prime}, \hat{\ell}_{a}^{\prime}\right)$ to be equivalent if they are related by the rescaling

$$
\begin{aligned}
\ell^{\prime a} & \widehat{=} e^{\sigma} \ell^{a}, \\
\kappa^{\prime} & \widehat{=} e^{\sigma}\left(\kappa+£_{\ell} \sigma\right), \\
\hat{\ell}_{a}^{\prime} & =e^{\sigma} \hat{\ell}_{a},
\end{aligned}
$$

where $\sigma$ is a smooth function on $\mathcal{N}$. We denote by

$$
\mathfrak{p}=\left[\ell^{a}, \kappa, \hat{\ell}_{a}\right]
$$

the equivalence class associated with $\left(\ell^{a}, \kappa, \hat{\ell}_{a}\right)$. A choice of equivalence class is the desired boundary structure on $\mathcal{N}$.

It is clear that a choice of boundary structure $\mathfrak{p}=\left[\ell^{a}, \kappa, \hat{\ell}_{a}\right]$ determines a unique universal intrinsic structure $\mathfrak{u}$ : choose a representative $\left(\ell^{a}, \kappa, \hat{\ell}_{a}\right)$, discard $\hat{\ell}_{a}$, and note that from eq. (5.1) that $\ell^{a}$ can be regarded as an intrinsic vector field $\ell^{i}$. Then from $\left(\ell^{i}, \kappa\right)$ form the equivalence class $\mathfrak{u}=\left[\ell^{i}, \kappa\right]$ under the equivalence relation (4.1). From eqs. (4.1) and (5.2) the result is independent of the representative $\left(\ell^{a}, \kappa, \hat{\ell}_{a}\right)$ initially chosen. We will denote this induced intrinsic structure by $\mathfrak{u}(\mathfrak{p})$. Our boundary structures contain more information than the intrinsic structures, which will be necessary for the definition of the field configuration space. We will say that a boundary structure $\mathfrak{p}$ is complete if the corresponding intrinsic structure $\mathfrak{u}$ is complete.

In addition, a metric $g_{a b}$ on $M$ for which the boundary $\mathcal{N}$ is null determines a unique boundary structure $\mathfrak{p}$, just as for intrinsic structures discussed in section 4.1. Pick a normal covector $\hat{\ell}_{a}$ to $\mathcal{N}$, raise the index to obtain $\ell^{a}=g^{a b} \hat{\ell}_{b}$, and compute the non-affinity $\kappa$ using the metric via eq. (3.2). Then from the triple $\left(\ell^{a}, \kappa, \hat{\ell}_{a}\right)$ form the equivalence class $\mathfrak{p}=\left[\ell^{a}, \kappa, \hat{\ell}_{a}\right]$. The result is independent of the choice of initial normal covector, by the equivalence relation (5.2).

Given a boundary structure $\mathfrak{p}$, we now define the field configuration space $\mathscr{F}_{\mathfrak{p}}$ to be the set of smooth metrics $g_{a b}$ on $M$ which satisfy on $\mathcal{N}$ the relations

$$
\begin{aligned}
\ell^{a} & \widehat{=} g^{a b} \hat{\ell}_{b}, \\
\ell^{a} \nabla_{a} \ell^{b} & \widehat{=} \kappa \ell^{b} .
\end{aligned}
$$

\footnotetext{
${ }^{23}$ This normal covector was denoted $\ell_{a}$ earlier in the paper. We introduce the separate notation $\hat{\ell}_{a}$ because the context here of the definition of a boundary structure does not involve a metric, and to clarify that there are two independent tensor fields in the definition.
} 
From eqs. (5.1) and (5.4a) it follows that the boundary $\mathcal{N}$ is null with respect to $g_{a b}$, so that $\mathscr{F}_{\mathfrak{p}} \subset \mathscr{F}_{0}$. Also if the conditions (5.4) are satisfied by one representative $\left(\ell^{a}, \kappa, \hat{\ell}_{a}\right)$, they will be satisfied by all representatives, from eqs. (3.3) and (5.2). Hence $\mathscr{F}_{\mathfrak{p}}$ is well defined and depends only on $\mathfrak{p}$. (An equivalent definition of $\mathscr{F}_{\mathfrak{p}}$ is the set of smooth metrics on $M$ for which $\mathcal{N}$ is null and whose associated boundary structures agree with $\mathfrak{p}$ ). We define the corresponding covariant phase space $\overline{\mathscr{F}}_{\mathfrak{p}}$ to be the set of metrics in $\mathscr{F}_{\mathfrak{p}}$ which satisfy the equations of motion.

Note that the order of definitions being used in this construction is the opposite of that which is normally used. Normally, one first picks the spacetime metric, then defines the covariant version of the null normal by raising the index as in eq. (5.4a), and defines the non-affinity function $\kappa$ via eq. (5.4b). Here, instead, we first choose the quantities $\ell^{a}$, $\hat{\ell}_{a}$, and $\kappa$, and then specialize the spacetime metric $g_{a b}$ to enforce eqs. (5.4).

It may appear that the conditions (5.4) we are imposing on the metric are overly restrictive. In fact, they do not restrict the physical degrees of freedom in the sense that $\mathscr{F}_{\mathfrak{p}}$ is obtained from $\mathscr{F}_{0}$ by a fixing of the diffeomorphism freedom. More precisely, given a complete boundary structure $\mathfrak{p}$, and given any metric $g_{a b}$ on $M$ for which $\mathcal{N}$ is null and for which the boundary structure induced by $g_{a b}$ is complete, one can find a diffeomorphism $\psi: M \rightarrow M$ which takes $\mathcal{N}$ into $\mathcal{N}$ for which $\psi_{*} g_{a b}$ satisfies the conditions (5.4). This is proved in appendix B.

We next show that the mapping $\mathfrak{p} \rightarrow \mathscr{F}_{\mathfrak{p}}$ is injective, so that if $\mathscr{F}_{\mathfrak{p}}=\mathscr{F}_{\mathfrak{p}^{\prime}}$ then $\mathfrak{p}=\mathfrak{p}^{\prime}$. This property will be used in section 5.2 below. Let $\left(\ell^{a}, \kappa, \hat{\ell}_{a}\right)$ be a representative of $\mathfrak{p}$, and $\left(\ell^{\prime a}, \kappa^{\prime}, \hat{\ell}_{a}^{\prime}\right)$ be a representative of $\mathfrak{p}^{\prime}$. Since $\hat{\ell}_{a}$ and $\hat{\ell}_{a}^{\prime}$ are both normals to $\mathcal{N}$, they are related by a rescaling, and hence by adjusting our choice of representative we can without loss of generality take $\hat{\ell}_{a}=\hat{\ell}_{a}^{\prime}$. Now pick a metric $g_{a b}$ which belongs to both $\mathscr{F}_{\mathfrak{p}}$ and $\mathscr{F}_{\mathfrak{p}^{\prime}}$. Applying eq. (5.4a) to both $\mathfrak{p}$ and $\mathfrak{p}^{\prime}$ we find that $\ell^{a}=\ell^{\prime a}$, and it follows from eq. (5.4b) that $\kappa=\kappa^{\prime}$. Hence we have $\mathfrak{p}=\mathfrak{p}^{\prime}$.

\subsection{Symmetry algebra of the field configuration space}

We now show that for a complete boundary structure $\mathfrak{p}$, the symmetry algebra associated with the field configuration space $\mathscr{F}_{\mathfrak{p}}$ coincides with the algebra $\mathfrak{g}_{\mathfrak{u}}$ of the universal intrinsic structure $\mathfrak{u}$ of the null surface discussed in section 4 , where $\mathfrak{u}=\mathfrak{u}(\mathfrak{p})$ is the intrinsic structure obtained from $\mathfrak{p}$ discussed in section 5.1.

We start by defining the group of diffeomorphisms on $M$ whose pullbacks preserve the boundary and the field configuration space:

$$
H_{\mathfrak{p}}=\left\{\psi: M \rightarrow M \mid \psi(\mathcal{N})=\mathcal{N}, \psi_{*} \mathscr{F}_{\mathfrak{p}}=\mathscr{F}_{\mathfrak{p}}\right\}
$$

These diffeomorphisms induce diffeomorphisms of the boundary: for any $\psi$ in $H_{\mathfrak{p}}$ we define

$$
\varphi=\left.\psi\right|_{\mathcal{N}},
$$

and since $\psi$ preserves the boundary, $\varphi$ is a diffeomorphism from $\mathcal{N}$ to $\mathcal{N}$. Next, since $\psi$ preserves the boundary, the pullback of the normal must be a rescaling of the normal, so we have

$$
\psi_{*} \hat{\ell}_{a}=e^{\gamma} \hat{\ell}_{a}
$$


where $\gamma=\gamma\left(\psi, \hat{\ell}_{a}\right)$ is a smooth function on $\mathcal{N}$ which depends on the diffeomorphism and on the normalization of the normal covector $\hat{\ell}_{a}$. From eq. (5.7) we find for the dependence on the normalization [cf. eq. (4.6) above]

$$
\gamma\left(\psi, e^{\sigma} \hat{\ell}_{a}\right)=\gamma\left(\psi, \hat{\ell}_{a}\right)+\psi_{*} \sigma-\sigma
$$

for any smooth function $\sigma$ on $\mathcal{N}$.

Next from the definition (5.5) we have

$$
\mathscr{F}_{\mathfrak{p}}=\psi_{*} \mathscr{F}_{\mathfrak{p}}=\mathscr{F}_{* \mathfrak{p}},
$$

where the action of the pullback $\psi_{*}$ on the boundary structure $\mathfrak{p}$ is defined by its action on a representative $\left(\ell^{a}, \kappa, \hat{\ell}_{a}\right)$. Now using the injectivity property of the mapping $\mathfrak{p} \rightarrow \mathscr{F}_{\mathfrak{p}}$ proved in section 5.1 , we obtain

$$
\psi_{*} \mathfrak{p}=\mathfrak{p} .
$$

From the definition (5.2) of the equivalence class, it follows that for a given representative $\left(\ell^{a}, \kappa, \hat{\ell}_{a}\right)$ in $\mathfrak{p}$, the pullback $\psi_{*}$ acts as a scaling transformation for some smooth scaling function $\beta=\beta(\psi)$ on $\mathcal{N}$ [cf. eq. (4.4) above]:

$$
\begin{aligned}
\psi_{*} \ell^{a} & \widehat{=} e^{\beta} \ell^{a}, \\
\psi_{*} \kappa & \widehat{=} e^{\beta}\left(\kappa+£_{\ell} \beta\right), \\
\psi_{*} \hat{\ell}_{a} & \widehat{=} e^{\beta} \hat{\ell}_{a} .
\end{aligned}
$$

In the first two of these equations we can replace $\ell^{a}$ with $\ell^{i}$, by eq. (5.1), and we can replace $\psi_{*}$ with $\varphi_{*}$. These two equations then coincide with the defining equations (4.4) for the group $G_{\mathfrak{u}}$ of boundary symmetries $\varphi: \mathcal{N} \rightarrow \mathcal{N}$ that preserve the intrinsic structure $\mathfrak{u}$ associated with $\mathfrak{p}$. Combining eqs. (5.7) and (5.11c) yields that

$$
\gamma(\psi)=\beta(\varphi)
$$

Hence we have shown that

$$
H_{\mathfrak{p}}=\left\{\psi: M \rightarrow M \mid \psi(\mathcal{N})=\mathcal{N}, \varphi \in G_{\mathfrak{u}}, \beta(\varphi)=\gamma(\psi)\right\},
$$

where $\varphi$ is the diffeomorphism (5.6) induced on the boundary. A bulk diffeomorphism $\psi$ is a symmetry if it preserves the boundary, if the induced boundary diffeomorphism is a symmetry of the intrinsic structure on the boundary, and if the scaling function $\gamma(\psi)$ defined by eq. (5.7) satisfies eq. (5.12).

We next specialize these results to infinitesimal diffeomorphisms. Linearized diffeomorphisms on $M$ are parameterized in terms of vector fields $\xi^{a}$ on $M$, and the boundary is preserved if these vector fields are tangent to the boundary,

$$
\xi^{a} \hat{\ell}_{a} \widehat{=} 0
$$

We define

$$
\vec{\chi}=\left.\vec{\xi}\right|_{\mathcal{N}}
$$


and it follows from the condition (5.14) that we can regard $\vec{\chi}$ as an intrinsic vector field $\chi^{i}$ on $\mathcal{N}$, as in section 4.3 above. The definition (5.7) of the scaling function $\gamma$ becomes

$$
£_{\xi} \hat{\ell}_{a} \widehat{=} \gamma\left(\xi^{a}, \hat{\ell}_{a}\right) \hat{\ell}_{a},
$$

while the dependence (5.8) on the normalization of the normal becomes

$$
\gamma\left(\xi^{a}, e^{\sigma} \hat{\ell}_{a}\right)=\gamma\left(\xi^{a}, \hat{\ell}_{a}\right)+£_{\xi} \sigma
$$

The linearized version of the constraint (5.12) is

$$
\gamma\left(\xi^{a}\right)=\beta\left(\chi^{i}\right)
$$

Defining $\mathfrak{h}_{\mathfrak{p}}$ to be the Lie algebra corresponding to the group $H_{\mathfrak{p}}$, we find by linearizing the result (5.13) that

$$
\mathfrak{h}_{\mathfrak{p}}=\left\{\xi^{a} \text { on } M \mid \xi^{a} \hat{\ell}_{a} \widehat{=} 0, \chi^{i} \in \mathfrak{g}_{\mathfrak{u}}, \beta\left(\chi^{i}\right)=\gamma\left(\xi^{a}\right)\right\}
$$

where $\chi^{i}$ is given by the restriction (5.15) to the boundary and $\beta\left(\chi^{i}\right)$ is defined by eq. (4.11a).

Finally, to obtain the physical symmetry algebra, we need to factor out the trivial diffeomorphisms for which the symmetry generator charge variation (2.13) vanishes, using the equivalence relation $\sim$ defined in eq. (2.18). In section 6 below we compute the charge variation (2.13) explicitly, and in appendix $\mathrm{C}$ we show that it vanishes for all metric perturbations if and only if $\chi^{i}$ and $\gamma\left(\xi^{a}\right)$ both vanish. Hence the quotient set $\mathfrak{h}_{\mathfrak{p}} / \sim$ is parameterized by $\chi^{i}$ and $\gamma$, but from eq. (5.18) $\gamma$ is determined by $\chi^{i}$. We conclude from eq. (5.13) that

$$
\mathfrak{h}_{\mathfrak{p}} / \sim \cong \mathfrak{g}_{\mathfrak{u}}
$$

as claimed.

To summarize, infinitesimal symmetries are in one-to-one correspondence with symmetries $\chi^{i} \in \mathfrak{g}_{\mathfrak{u}}$ of the intrinsic structure $\mathfrak{u}$. However, all representatives $\xi^{a}$ whose restriction to the boundary is $\chi^{i}$ must also obey the constraint (5.18).

\subsection{Boundary conditions on the variation of the metric}

In our application of the Wald-Zoupas formalism we will need to consider variations of the metric of the form

$$
g_{a b} \rightarrow g_{a b}+\delta g_{a b}=g_{a b}+h_{a b}
$$

We assume that both the original and varied metric lie in the configuration space $\mathscr{F}_{\mathfrak{p}}$, so that they both satisfy conditions (5.4). In this subsection, we will derive some resulting boundary conditions on the metric variation $h_{a b}$ that will be useful in later sections of the paper. Specifically these conditions are

$$
\begin{aligned}
h_{a b} \ell^{b} & \widehat{=} 0 \\
\nabla_{c}\left(h_{a b} \ell^{a} \ell^{b}\right) & \widehat{=} 0 .
\end{aligned}
$$


Note that the condition (5.22b) is independent of the definition of $\ell^{a}$ off the surface, because of eq. (5.22a). As a consistency check of our computations, we show in appendix D that the conditions (5.22) are automatically satisfied for a metric perturbation of the form

$$
h_{a b}=£_{\xi} g_{a b}
$$

generated by a representative $\xi^{a}$ of a symmetry in the algebra discussed in the previous section.

We now turn to the derivation of eqs. (5.22). Equation (5.22a) follows from taking the variation of eq. (5.4a) and noting that $\ell^{a}$ and $\hat{\ell}_{a}$ are fixed under the variation. By varying the definition $(5.4 \mathrm{~b})$ of non-affinity and noting that $\kappa$ is fixed under the variation we find

$$
\nabla_{a} h_{b c} \ell^{a} \ell^{c}-\nabla_{b} h_{a c} \ell^{a} \ell^{c} / 2=0 .
$$

We can rewrite the first term as $\ell^{a} \nabla_{a}\left(\ell^{c} h_{b c}\right)-\left(\ell^{a} \nabla_{a} \ell^{c}\right) h_{b c}$. The first term here vanishes by eq. (5.22a) since the derivative is along the surface $\mathcal{N}$, while the second vanishes by eqs. (5.4b) and (5.22a). Thus we obtain $\nabla_{b} h_{a c} \ell^{a} \ell^{c}=0$, which is equivalent to eq. (5.22b) by eq. (5.22a).

It follows from eq. (5.22a) that we can regard $h^{a b}$ restricted to $\mathcal{N}$ as an intrinsic tensor $h^{i j}$ on $\mathcal{N}$. We can also construct the down index versions

$$
\begin{aligned}
h_{i}{ }^{j} & =q_{i k} h^{k j} \\
h_{i j} & =q_{i k} q_{j l} h^{k l}=\Pi_{i}^{a} \Pi_{j}^{b} h_{a b} .
\end{aligned}
$$

These quantities satisfy

$$
h_{i j} \ell^{i} \widehat{=} h_{i}^{j} \ell^{i} \widehat{=} 0
$$

from eqs. (3.7) and (5.22a). In four spacetime dimensions, $h^{i j}$ contains six independent components, $h_{i}^{j}$ five, and $h_{i j}$ three. We will express charge variations in section 6 below in terms of $h_{i}{ }^{j}$.

A useful quantity involving the metric perturbation that will appear in the charge variations can be defined as follows. Defining $h_{a}=h_{a}{ }^{b} \hat{\ell}_{b}$, we have from eqs. (5.4a) and (5.22a) that $h_{a}$ vanishes on $\mathcal{N}$. Hence there exists a one-form $\Gamma_{a}$ on $\mathcal{N}$ so that

$$
\nabla_{[a} h_{b]} \widehat{=} \hat{\ell}_{[a} \Gamma_{b]} .
$$

The quantity $\Gamma_{a}$ depends linearly on $h_{a}{ }^{b}$ and its first derivatives, including in directions off the surface $\mathcal{N}$, but is independent of the background metric and connection. It does depend on how one extends the definition of $\hat{\ell}_{a}$ off the surface $\mathcal{N}$. However if we impose on this extension the condition

$$
\nabla_{[a} \hat{\ell}_{b]} \widehat{=} 0,
$$

then $\Gamma_{a}$ is uniquely determined. From eq. (5.22b) it satisfies

$$
\Gamma_{a} \ell^{a}=0
$$

It is invariant under a rescaling of the normal $\ell^{a} \rightarrow e^{\sigma} \ell^{a}$. We also define the pullback $\Gamma_{i}=\Pi_{i}^{a} \Gamma_{a}$. 


\section{Global and localized charges for a null boundary component}

From the perspective of the covariant phase space, we have seen in the last two sections that general relativity in the presence of null boundaries has quite a rich structure as encapsulated in the infinite dimensional symmetry algebra $\mathfrak{g}_{\mathfrak{u}}$. With these symmetries at hand, in this section we move on to the calculation of the corresponding charges and fluxes. We compute the Noether charge $Q_{\xi}$ in section 6.1, its variation $\delta Q_{\xi}$ in section 6.2, the boundary symmetry generator $\mathcal{Q}_{\xi}$ and its variation in section 6.3 , and the localized charge or Wald-Zoupas charge $\mathcal{Q}_{\xi}^{\text {loc }}$ and its flux in section 6.4.

Appendix $\mathrm{H}$ computes the corresponding charges for a modified definition of field configuration space. As mentioned in the previous section the drawback of the modification is that one looses uniqueness in the prescription for defining localized charges.

\subsection{Noether charge}

For general relativity in vacuum, the Lagrangian, presymplectic potential 3 -form and Noether charge 2 -form are given by [6]

$$
\begin{aligned}
L_{a b c d} & =\frac{1}{16 \pi} \varepsilon_{a b c d} R, \\
\theta_{a b c} & =\frac{1}{16 \pi} \varepsilon_{a b c}{ }^{d}\left(g^{e f} \nabla_{d} h_{e f}-\nabla^{e} h_{d e}\right), \\
Q_{\xi a b} & =-\frac{1}{16 \pi} \varepsilon_{a b c d} \nabla^{c} \xi^{d} .
\end{aligned}
$$

The ambiguity (2.33) in the presymplectic potential can be resolved in the case of general relativity by demanding that the total number of derivatives of the metric $g_{a b}$ or metric perturbation $h_{a b}$ in the 2-form $Y_{a b}$ be two less than the number of derivatives appearing in the Lagrangian. One can readily convince oneself that there is no 2-form $Y_{a b}$ that depends on $g_{a b}, \varepsilon_{a b c d}$ and $h_{a b}$ that depends linearly on $h_{a b}$ and has no derivatives.

We now evaluate the pullback of the Noether charge 2 -form to $\mathcal{N}$. From eqs. (3.20) and (3.6) we find

$$
Q_{\xi i j}=-\frac{1}{16 \pi} \Pi_{i}^{a} \Pi_{j}^{b}\left(\bar{\varepsilon}_{a b c} \hat{\ell}_{d}-\bar{\varepsilon}_{d a b} \hat{\ell}_{c}+\bar{\varepsilon}_{c d a} \hat{\ell}_{b}-\bar{\varepsilon}_{b c d} \hat{\ell}_{a}\right) \nabla^{c} \xi^{d}=-\frac{1}{16 \pi} \Pi_{i}^{a} \Pi_{j}^{b} \bar{\varepsilon}_{a b c} q^{c}
$$

where $q^{c}=2 \hat{\ell}_{d} \nabla^{[c} \xi^{d]}$. Since $\hat{\ell}_{a} q^{a}=0$ it follows from eqs. (3.7) and (3.21) that we can rewrite this expression in terms of tensors intrinsic to $\mathcal{N}$.

$$
Q_{\xi i j}=-\frac{1}{16 \pi} \varepsilon_{i j k} q^{k}
$$

We can rewrite $q^{c}$ as

$$
q^{c}=g^{c d} £_{\xi} \hat{\ell}_{d}+£_{\xi} \ell^{c}-2 \xi^{b} \nabla_{b} \ell^{c},
$$

where we have used the validity of eq. (5.4a) on $\mathcal{N}$ and the fact that $\xi^{b} \nabla_{b}$ differentiates along the surface, by eq. (5.14). Next, using the definitions (4.11a) and (5.7) of $\beta$ and $\gamma$ and the condition (5.18) we obtain

$$
q^{c}=(\beta+\gamma) \ell^{c}-2 \xi^{b} \nabla_{b} \ell^{c}=2 \beta \ell^{c}-2 \xi^{b} \nabla_{b} \ell^{c} .
$$


From eqs. (3.7) and (5.14) the contracted $b$ index in the second term can be replaced by an intrinsic index $k$, and we can then use the definition (3.16) of the Weingarten map. Inserting the result into (6.3) and using (5.15) gives

$$
Q_{\xi i j}=\frac{1}{8 \pi} \varepsilon_{i j k}\left[\chi^{l} \mathcal{K}_{l}^{k}-\beta\left(\chi^{i}\right) \ell^{k}\right] .
$$

This expression is invariant under the scaling transformation (4.1), from the transformation properties $(3.24 \mathrm{c}),(4.12)$ and $(3.24 \mathrm{e})$.

Suppose now that we are given a cross section $\mathcal{S}$ of $\mathcal{N}$. The Noether charge associated with that cross section is given by integrating the two form (6.6). Letting $n_{i}$ denote the unique normal covector to $\mathcal{S}$ in $\mathcal{N}$ with the normalization (4.28), we obtain from the definition (3.23)

$$
Q_{\xi}(\mathcal{S})=\int_{\mathcal{S}} \boldsymbol{Q}_{\xi}=\frac{1}{8 \pi} \int_{\mathcal{S}} \varepsilon_{i j}\left[\chi^{l} n_{k} \mathcal{K}_{l}^{k}+\beta\left(\chi^{i}\right)\right]
$$

\subsection{Variation of Noether charge}

We next turn to computing the variation of the Noether charge under a variation of the metric of the form (5.21). Of all the quantities which appear in the expression (6.6) for the pullback of the Noether charge two-form, only the volume form $\varepsilon_{i j k}$ and the Weingarten map $\mathcal{K}_{l}{ }^{k}$ vary as the metric is varied. Using $\delta \varepsilon_{a b c d}=h \varepsilon_{a b c d} / 2$ with $h=g^{a b} h_{a b}=q^{A B} \delta q_{A B}$ we obtain

$$
16 \pi \delta Q_{\xi i j}=\varepsilon_{i j k}\left[h \chi^{l} \mathcal{K}_{l}^{k}-h \beta\left(\chi^{i}\right) \ell^{k}+2 \chi^{l} \delta \mathcal{K}_{l}^{k}\right] .
$$

To compute the variation of the Weingarten map we define $K_{a}{ }^{b}=\nabla_{a} \ell^{b}$, which when we pullback the $a$ index is orthogonal to $\hat{\ell}_{b}$ on the $b$ index and reduces to $\mathcal{K}_{l}{ }^{k}$. Taking a variation we find

$$
2 \Pi_{i}^{a} \delta K_{a}^{b}=\Pi_{i}^{a}\left[-\nabla^{b} h_{a c}+\nabla_{a} h_{c}^{b}+\nabla_{c} h_{a}^{b}\right] \ell^{c} .
$$

We now use the definition (5.27) of $\Gamma_{a}$ to rewrite the first two terms in (6.9), and rewrite the last term in terms of a Lie derivative. This yields

$$
2 \Pi_{i}^{a} \delta K_{a}^{b}=\Pi_{i}^{a}\left[\hat{\ell}_{a} \Gamma^{b}-\Gamma_{a} \hat{\ell}^{b}-h^{b c} \nabla_{a} \hat{\ell}_{c}+h_{a}{ }^{c} \nabla^{b} \hat{\ell}_{c}+£_{\ell} h_{a}^{b}+h_{a}^{c} \nabla_{c} \ell^{b}-h_{c}^{b} \nabla_{a} \ell^{c}\right] .
$$

The first term here vanishes by eq. (3.6). We can replace the $\hat{\ell}^{a}$ with $\ell^{a}$ in the second and third terms, using the condition (5.4a) and the fact that the derivative is along the surface. We rewrite the fourth term using the condition (5.28) as $h_{a}{ }^{c} \nabla_{c} \hat{\ell}^{b}=h_{a}{ }^{c} \nabla_{c} \ell^{b}$, where we have used the fact that the derivative is along the surface by eq. (5.22a). We thus obtain

$$
2 \Pi_{i}^{a} \delta K_{a}^{b}=\Pi_{i}^{a}\left[-\Gamma_{a} \ell^{b}+£_{\ell} h_{a}^{b}+2 h_{a}^{c} \nabla_{c} \ell^{b}-2 h_{c}^{b} \nabla_{a} \ell^{c}\right]
$$

Now the individual terms on the right hand side are all orthogonal ${ }^{24}$ to $\hat{\ell}_{b}$, by eqs. (5.1) and (5.22a). Hence they all give rise to tensors intrinsic to $\mathcal{N}$. Also the contractions on

\footnotetext{
${ }^{24}$ For the second term this is because $\hat{\ell}_{b} £_{\ell} h_{a}{ }^{b}=£_{\ell}\left(h_{a}{ }^{b} \hat{\ell}_{b}\right)-h_{a}{ }^{b} £_{\ell} \hat{\ell}_{b}=-h_{a}{ }^{b} \ell^{c} £_{\ell} g_{b c}=-h_{a}{ }^{b} \nabla_{b}\left(\ell_{c} \ell^{c}\right) / 2-$ $\kappa h_{a}{ }^{b} \ell_{b}=0$.
} 
the $c$ index in the last two terms can be replaced by intrinsic contractions, by eqs. (3.7) and (5.22a). Using the definition (3.16) finally gives

$$
\delta \mathcal{K}_{i}{ }^{j}=-\frac{1}{2} \Gamma_{i} \ell^{j}+\frac{1}{2} £_{\ell} h_{i}{ }^{j}+h^{k}{ }_{i} \mathcal{K}_{k}{ }^{j}-h^{j}{ }_{k} \mathcal{K}_{i}{ }^{k} .
$$

From eq. (6.8) we obtain for the variation of the pullback of the Noether charge two-form

$$
\delta Q_{\xi i j}=\frac{1}{16 \pi} \varepsilon_{i j k}\left[h \chi^{l} \mathcal{K}_{l}{ }^{k}-h \beta\left(\chi^{i}\right) \ell^{k}-\chi^{l} \Gamma_{l} \ell^{k}+\chi^{l} £_{\ell} h_{l}{ }^{k}+2 \chi^{l} h^{m}{ }_{l} \mathcal{K}_{m}{ }^{k}-2 \chi^{l} h^{k}{ }_{m} \mathcal{K}_{l}{ }^{m}\right] .
$$

\subsection{Global charges that generate boundary symmetries}

As described in section 2.4 above, the charge $\mathcal{Q}_{\xi}$ that generates a boundary symmetry $\xi^{a}$ has a variation $\delta \mathcal{Q}_{\xi}$ which is an integral of the form (2.12) over a Cauchy surface $\Sigma$, and can be expressed as the surface integral (2.13) over the boundary $\partial \Sigma$. We now assume that a cross section $\mathcal{S}$ of the null surface $\mathcal{N}$ is a component of the boundary $\partial \Sigma$. This gives

$$
\delta \mathcal{Q}_{\xi}=\int_{\mathcal{S}} \delta \mathcal{Q}_{\xi} a b+\ldots
$$

where the ellipses represent integrals over the remaining components of $\partial \Sigma$ (for example spatial infinity). Here the integrand is the two-form

$$
\delta \mathcal{Q}_{\xi a b}=\delta Q_{\xi a b}-\xi^{c} \theta_{c a b}
$$

and $\theta_{a b c}$ is the presymplectic potential three-form (6.1b). Pulling back this expression to $\mathcal{N}$ using eqs. (3.20) and (3.21) gives

$$
\theta_{i j k}=\frac{1}{16 \pi} \varepsilon_{i j k} \ell^{f}\left(\nabla_{f} h-\nabla_{e} h_{f}^{e}\right) .
$$

The second term can be rewritten using the boundary conditions (5.22a) and (5.22b) as $-l^{f} \nabla_{e} h_{f}^{e}=h_{f}^{e} \nabla_{e} \ell^{f}$, which then allows using the definition (3.16). This yields

$$
\theta_{i j k}=\frac{1}{16 \pi} \varepsilon_{i j k}\left[£_{\ell} h+h_{i}{ }^{j} \mathcal{K}_{j}{ }^{i}\right] .
$$

In this expression the trace $h$ of the metric perturbation can be written as $q^{A B} h_{A B}$, from the condition (5.22a), while the second term in the brackets can be written as $^{25}$

$$
h_{A B} \mathcal{K}^{A B}=h_{A B}\left(\frac{1}{2} \theta q^{A B}+\sigma^{A B}\right),
$$

from eqs. (3.8), (3.13), (3.14), (3.19) and (5.26). Finally using eqs. (6.13) and (6.17) yields for the pullback of the perturbed symmetry generator two-form (6.15)

$$
\begin{aligned}
\delta \mathcal{Q}_{\xi i j}= & \frac{1}{16 \pi} \varepsilon_{i j k}\left[h \chi^{l} \mathcal{K}_{l}{ }^{k}-h \beta\left(\chi^{i}\right) \ell^{k}-\chi^{l} \Gamma_{l} \ell^{k}+\chi^{l} £_{\ell} h_{l}{ }^{k}+2 \chi^{l} h^{m}{ }_{l} \mathcal{K}_{m}{ }^{k}\right. \\
& \left.-2 \chi^{l} h^{k}{ }_{m} \mathcal{K}_{l}{ }^{m}-\chi^{k} £_{\ell} h-\chi^{k} h_{i}{ }^{j} \mathcal{K}_{j}{ }^{i}\right] .
\end{aligned}
$$

\footnotetext{
${ }^{25}$ An alternative form is $h_{i j} K_{k l} q^{i k} q^{j l}$ where $q^{i j}$ is any tensor that satisfies $q^{i j} q_{i k} q_{j l}=q_{k l}$, from eq. (3.8).
} 
In general this expression is not a total variation, and so cannot be integrated up to compute a finite charge corresponding to the first term in the symmetry generator (6.14). To see this, we compute the pullback $(2.15)$ to $\mathcal{N}$ of the presymplectic current, contracted with $\chi^{i}$. As shown in section 2 above, when this quantity is nonzero the variation (6.19) is not a total variation. Taking a variation of the expression (6.17) for the pullback of the presymplectic potential, and using the formula (6.12) for the variation of the Weingarten map, we obtain

$$
\begin{aligned}
\chi^{i} \omega_{i j k}\left(h^{l m}, h^{\prime p q}\right)= & \frac{1}{16 \pi} \chi^{i} \varepsilon_{i j k}\left[\frac{1}{2} h £_{\ell} h^{\prime}+\frac{1}{2} £_{\ell} h_{m}{ }^{l} h_{l}^{\prime}{ }^{m}+\frac{1}{2} h h_{l}^{\prime}{ }^{m} \mathcal{K}_{m}{ }^{l}+2 h_{m}{ }^{p} h_{l}^{\prime}{ }^{m} \mathcal{K}_{p}{ }^{l}\right] \\
& -\left(h \leftrightarrow h^{\prime}\right) .
\end{aligned}
$$

The integral of this quantity over a cross section $\mathcal{S}$ is nonvanishing in general. It does vanish in the case when $\chi^{i}$ is tangent to $\mathcal{S}$, that is, when it is a generator of the diffeomorphism symmetries. It also vanishes if we demand that both the background and perturbed configurations are shear and expansion free on $\mathcal{S}$, and in particular if they are stationary on $\mathcal{S}$.

We now specialize to the case where the null surface $\mathcal{N}$ is the future event horizon $\mathcal{H}^{+}$ of a black hole. The boundary of the horizon consists of the asymptotic boundary $\mathcal{H}_{+}^{+}$at future timelike infinity, together with a bifurcation twosphere $\mathcal{H}_{-}^{+}$in the case of an eternal black hole. In appendix $\mathrm{G}$ we show that the obstruction (6.20) vanishes on the bifurcation twosphere $\mathcal{H}_{-}^{+}$. We also show that the obstruction vanishes on the future boundary $\mathcal{H}_{+}^{+}$, assuming certain fall off conditions on the shear along the horizon towards future timelike infinity, and we argue that these fall off conditions are physically reasonable. Hence for horizons, eq. (6.14) can be used directly to compute the contribution from the horizon to global charges.

We also show in appendix $\mathrm{G}$ that the correction term $i_{\xi} \boldsymbol{\Theta}$ in the definition (2.25) of the localized charge vanishes on the boundaries $\mathcal{H}_{ \pm}^{+}$, under the same assumptions as above. Hence from eq. (2.32) the contribution from the horizon to the global charge can be written as

$$
\mathcal{Q}_{\xi}^{\text {loc }}\left(\mathcal{H}_{+}^{+}\right)-\mathcal{Q}_{\xi}^{\text {loc }}\left(\mathcal{H}_{-}^{+}\right)
$$

Here $\mathcal{Q}_{\xi}^{\text {loc }}$ is the localized charge which is computed explicitly in the next subsection, cf. eq. (6.27).

Global conservation laws involving these global charges are discussed further in section 7 below.

\subsection{Localized (Wald-Zoupas) charges and fluxes}

We now turn to a computation of localized charges $\mathcal{Q}_{\xi}^{\text {loc }}(\mathcal{S})$ for cross sections $\mathcal{S}$ of a null surface $\mathcal{N}$. As explained in section 2 above, the integrand in the expression (2.25) for this charge is given by adding to the pullback of the right hand side of eq. (6.15) a term $\chi^{k} \Theta_{i j k}$, where the presymplectic potential $\Theta_{i j k}$ is of the form [cf. eq. (2.30)]

$$
\Theta_{i j k}=\theta_{i j k}-\delta \alpha_{i j k}
$$


that is necessary for the right hand side to be a total variation. In addition, $\Theta_{i j k}$ is required to have the property that it vanish on backgrounds for which the null surface is shear free and expansion free. Then the charge is given by the expression (2.22) where the integrand is

$$
\mathcal{Q}_{\xi i j}^{\text {loc }}=Q_{\xi i j}-\chi^{k} \alpha_{i j k}
$$

up to an overall constant of integration on phase space. We verify that this constant of integration vanishes by showing that the right hand side of eq. (6.23) vanishes on our reference solution, and by assuming that the left hand side vanishes on this solution. This computation is carried out in appendix E.

We choose the 3 -form $\boldsymbol{\alpha}$ on $\mathcal{N}$ given by

$$
\alpha_{i j k}=\frac{1}{8 \pi} \theta \varepsilon_{i j k}
$$

where $\theta$ is the expansion (3.28). Computing its variation yields

$$
\delta \alpha_{i j k}=\frac{1}{16 \pi} \varepsilon_{i j k}\left(h \theta+£_{\ell} h\right) .
$$

Combining this with eqs. (6.17), (6.22) and (6.18) gives for the presymplectic potential on $\mathcal{N}$

$$
\Theta_{i j k}=\frac{1}{16 \pi} \varepsilon_{i j k}\left[h_{i}{ }^{j} \mathcal{K}_{j}{ }^{i}-h \theta\right]=\frac{1}{16 \pi} \varepsilon_{i j k} h^{A B}\left(\sigma_{A B}-\frac{1}{2} q_{A B} \theta\right) .
$$

This choice of presymplectic potential on a null surface was independently previously suggested in eq. (8.2.20) of a thesis by Morales [64]. For backgrounds for which the null surface is shear free and expansion free, it follows from eq. (6.26) that $\Theta$ vanishes, as required. The two-form (6.23) is now obtained by combining eqs. (6.6) and (6.24), which gives

$$
\mathcal{Q}_{\xi i j}^{\text {loc }}=\frac{1}{8 \pi} \varepsilon_{i j k}\left[\chi^{l} \mathcal{K}_{l}^{k}-\theta \chi^{k}-\beta\left(\chi^{i}\right) \ell^{k}\right] .
$$

It follows from the transformation properties (3.24) and (4.12) that this two-form is invariant under the rescaling (3.1).

We next argue that the expression (6.27) we have derived for the localized charge is unique. As discussed in section 2.7 above, the presymplectic potential $\Theta$ will be unique if there does not exist a 3 -form $\boldsymbol{W}(\phi)$ on the boundary $\mathcal{N}$ that is locally and covariantly constructed out of the fields and of the universal structure, with the property that its variation $\delta \boldsymbol{W}$ vanishes identically on solutions for which the null boundary is shear free and expansion free. We assume that $\boldsymbol{W}$ depends analytically on the fields, and that the maximum number of derivatives in the expression for $\boldsymbol{W}$ is one less than the number of derivatives appearing in the Lagrangian, or one in this case.

The various geometrical quantities on which $\boldsymbol{W}$ can depend are reviewed in section 3 above. The restriction on the number of derivatives in $\boldsymbol{W}$ eliminates other quantities, not reviewed in section 3 , that can be used to construct candidate expressions for $\boldsymbol{W}$, such as $\varepsilon_{i j k} £_{\ell} R$ where $R$ is the Ricci scalar. Using the finite number of quantities in section 3 one can show by inspection that there are no expressions with the right properties. For 
example the expressions $\kappa \varepsilon_{i j k}$ and $\varepsilon_{[i j} D_{k]} \theta$ not invariant under the transformation (3.24), while the expression $\left(\sigma_{A B} \sigma^{A B} / \theta\right) \varepsilon_{i j k}$ is invariant but does not depend analytically on the fields. We conclude that $\boldsymbol{W}=0$ and so $\boldsymbol{\Theta}$ and $\mathcal{Q}_{\xi}^{\text {loc }}(\mathcal{S})$ are uniquely determined by our assumptions and by the Lagrangian $\boldsymbol{L}$.

Finally, the on-shell flux $d \mathcal{Q}_{\xi}^{\text {loc }}$ associated with the localized charge is given by the symplectic potential $\boldsymbol{\Theta}$ evaluated at $h_{a b}=£_{\xi} g_{a b}$, from eq. (2.29). From the expression (6.26) for $\Theta$, combined with eqs. (3.7) and (3.16) to transform from three dimensional notation to four dimensional notation, we obtain

$$
\left(d \mathcal{Q}_{\xi}^{\text {loc }}\right)_{i j k}=\frac{1}{8 \pi} \varepsilon_{i j k} \nabla_{a} \xi_{b}\left(\nabla^{(a} \ell^{b)}-g^{a b} \theta\right) .
$$

Alternatively, the flux can be obtained by taking an exterior derivative of the twoform (6.27)

$$
\left(d \mathcal{Q}_{\xi}^{\text {loc }}\right)_{i j k}=\frac{1}{8 \pi} \varepsilon_{i j k} \hat{D}_{p}\left[\chi^{m} \mathcal{K}_{m}^{p}-\theta \chi^{p}-\beta \ell^{p}\right],
$$

where $\hat{D}_{p}$ is the divergence operator (3.25). It follows from the transformation properties (3.24) and (4.12) that this flux is invariant under the rescaling (3.1). We show in appendix F that the two expressions (6.28) and (6.29) for the flux coincide. This serves as a consistency check of the formalism.

\subsection{Charges and fluxes for specific symmetry generators}

We now specialize as before to a cross section $\mathcal{S}$ with normal $n_{i}$. For the special case of a supertranslation with $\chi^{k}=f \ell^{k}$, integrating the 2-form (6.27) over $\mathcal{S}$ and using eqs. (3.18), (4.19), (3.23) and (4.28) gives the charge

$$
\mathcal{Q}_{f}^{\text {loc }}(\mathcal{S})=\frac{1}{8 \pi} \int_{\mathcal{S}} \varepsilon_{i j}\left[\theta f-£_{\ell} f-\kappa f\right] .
$$

For stationary null surfaces, these charges vanish identically for affine supertranslations, for which $£_{\ell} f+\kappa f=0$. The corresponding flux through a region $\Delta \mathcal{N}$ of $\mathcal{N}$ is given by integrating the expression (6.29):

$$
\Delta \mathcal{Q}_{f}^{\text {loc }}=\frac{1}{8 \pi} \int_{\Delta \mathcal{N}} \varepsilon_{i j k} \hat{D}_{p}\left[\left(f \kappa-\theta f+£_{\ell} f\right) \ell^{p}\right] .
$$

This can be simplified using the formula (3.28), the symmetry condition (4.17) and Raychaudhuri's equation in vacuum to give

$$
\Delta \mathcal{Q}_{f}^{\text {loc }}=\frac{1}{8 \pi} \int_{\Delta \mathcal{N}} \varepsilon_{i j k} f\left(\theta \kappa-\theta^{2}-£_{\ell} \theta\right)=\frac{1}{8 \pi} \int_{\Delta \mathcal{N}} \varepsilon_{i j k} f\left(\sigma_{A B} \sigma^{A B}-\frac{1}{2} \theta^{2}\right) .
$$

We next consider $\operatorname{diff}(\mathcal{Z})$ generators of the form $\chi^{i}=X^{i}$ where $X^{i} n_{i}=0$, making use of the decomposition (4.29). Here $n_{i}$ is the normal to the cross section $\mathcal{S}$, and we also demand that it obey the differential equation (4.32) on $\mathcal{N}$, in order that $X^{i}$ be an element of the symmetry algebra $\mathfrak{g}_{\mathfrak{u}}$. For such generators the pullback to $\mathcal{S}$ of $\varepsilon_{i j k} X^{k}$ vanishes, 
and so from eqs. (6.23) and (6.24) the localized charge and Noether charge coincide. From eq. (6.27) the localized charge is

$$
\mathcal{Q}_{X}^{\text {loc }}(\mathcal{S})=\frac{1}{8 \pi} \int_{\mathcal{S}} \varepsilon_{i j}\left[X^{l} \mathcal{K}_{l}{ }^{k} n_{k}+\beta\right]
$$

and the corresponding flux from eq. (6.29) is

$$
\Delta \mathcal{Q}_{X}^{\text {loc }}=\frac{1}{8 \pi} \int_{\Delta \mathcal{N}} \varepsilon_{i j k} \hat{D}_{p}\left[X^{m} \mathcal{K}_{m}^{p}-\theta X^{p}-\beta \ell^{p}\right] .
$$

\subsection{Stationary regions of the null surface}

We now specialize to stationary regions of the null surface to obtain explicit forms for the various charges. In stationary regions the general charge (6.27) reduces to

$$
\mathcal{Q}_{\xi}^{\text {loc }}(\mathcal{S})=-\frac{1}{8 \pi} \int_{\mathcal{S}} \varepsilon_{i j}\left(\chi^{l} \omega_{l}-\beta\right),
$$

by eqs. (3.31), (A.13) and (4.28). The integrand here has a vanishing Lie derivative with respect to the Killing field $\tau^{i}$, so the result is independent of $\mathcal{S}$ as one would expect. To see this, take the Lie derivative of the integrand with respect to $\ell^{i}$, and simplify using eqs. (3.30), (4.11) and (A.10) to obtain $-\varepsilon_{i j}\left[\chi^{k} £_{\ell} \omega_{k}-£_{\chi} \kappa\right] /(8 \pi)$. Now specializing without loss of generality to the choice of representative $\ell^{i}=\tau^{i}$ and using eqs. (3.34) and (3.35) shows that the expression vanishes.

We next specialize to the choice of normal $\ell^{i}=\tau^{i}$, and to a coordinate system $\left(\tau, \theta^{A}\right)$ for which the Killing field is $\vec{\tau}=\partial / \partial \tau$, and we write the rotation one-form (3.33) and symmetry generator as

$$
\boldsymbol{\omega}_{\tau}=\omega_{\tau A}\left(\theta^{B}\right) d \theta^{A}+\kappa_{\tau} d \tau
$$

and

$$
\vec{\chi}=\left[\hat{\alpha}\left(\theta^{A}\right) e^{-\kappa_{\tau} \tau}+\hat{\beta}\left(\theta^{A}\right)\right] \frac{\partial}{\partial \tau}+X^{A}\left(\theta^{B}\right) \frac{\partial}{\partial \theta^{A}} .
$$

Here $\hat{\alpha}$ parameterizes the affine supertranslations, $\hat{\beta}$ the Killing supertranslations, ${ }^{26}$ and $X^{A}$ the $\operatorname{diff}\left(S^{2}\right)$ transformations or superrotations. From eq. (4.11a) we obtain $\beta=$ $\kappa_{\tau} \hat{\alpha} \exp \left[-\kappa_{\tau} \tau\right]$ and substituting into eq. (6.35) gives

$$
\mathcal{Q}_{\xi}^{\text {loc }}(\mathcal{S})=-\frac{1}{8 \pi} \int_{\mathcal{S}} \varepsilon_{i j}\left(X^{A} \omega_{\tau A}+\hat{\beta} \kappa_{\tau}\right)
$$

\section{Global conservation laws involving black holes}

Although our analysis has considered arbitrary null surfaces in the preceding sections, our main interest lies with black holes. Accordingly, in the next few sections, we take $\mathcal{N}$ to be the future event horizon $\mathcal{H}^{+}$of a black hole. This can either be a black hole formed in gravitational collapse, or an eternal black hole, as in figure 1 above. We note that our analysis is limited to smooth horizons, and that generic horizons are not smooth because

\footnotetext{
${ }^{26}$ Note that these parameters $\hat{\alpha}$ and $\hat{\beta}$ do not coincide with the parameters $\alpha$ and $\beta$ of eq. (4.8).
} 
of generators that join the horizon. We leave the analysis of charges and symmetries associated with nonsmooth horizons for future work.

In this section we consider global conservation laws involving black hole horizons. As discussed in the introduction, we distinguish between localized conservation laws that involve only one component $\mathcal{B}_{j}$ of the spacetime boundary, and global conservation laws that involve entire Cauchy surfaces. The foundation for both types of laws is the fact that the expression (2.12) for the variation of the global charge $\mathcal{Q}_{\xi}$ is invariant under local deformations of the hypersurface $\Sigma$ when on shell, from eq. (2.5). In situations where the charge variation is a total variation and the condition (2.15) is satisfied (which happens for internal symmetries), one does not need to distinguish between these two types of conservation laws. More generally, the localized conservation laws require the application of the Wald-Zoupas procedure, while the global laws do not, as argued in section 2.4 above.

As discussed in the introduction, in the past few years an infinite set of new global conservation laws in gauge theories have been discovered, associated with "large" gauge transformations which are not trivial at infinity $[4,7]$. Similar conservation laws have been argued for in the gravitational case [7, 65], although completely rigorous derivations have yet to be given. One of the key motivations for studying horizon symmetries and charges is the realization that the associated global conservation laws place constraints on black hole evaporation, and that the (electric parity superrotation) charges constitute "soft hair" that may play a role in how information is released as a black hole evaporates $[5,15,16$, $24,65,66]$ (see also [7] for a complete review). In this section we review the status of these conservation laws and the implications of our results for their formulation.

Consider for example a spacetime with no horizons for which the only components of the boundary are $\mathscr{I}^{+}, \mathscr{I}^{-}$and the points at infinity $i^{-}, i^{0}$ and $i^{+}$, and specialize to vacuum general relativity. Since the charge variation (2.12) is invariant under local deformations of the Cauchy surface $\Sigma$, one can deform $\Sigma$ into the distant past and also into the distant future. Then with appropriate sign conventions one obtains a conservation law of the form

$$
\delta \mathcal{Q}_{\xi}\left(\mathscr{I}^{-}\right)+\delta \mathcal{Q}_{\xi}\left(i^{-}\right)+\delta \mathcal{Q}_{\xi}\left(i^{0}\right)=\delta \mathcal{Q}_{\xi}\left(\mathscr{I}^{+}\right)+\delta \mathcal{Q}_{\xi}\left(i^{+}\right),
$$

where each term is an integral of the form (2.12) over the corresponding hypersurface or an appropriate limit of such integrals converging to one of the points at infinity, assuming such limits exist. If we specialize to spacetimes for which the Bondi mass vanishes at $\mathscr{I}_{+}^{+}$, the future limit of $\mathscr{I}^{+}$, and at $\mathscr{I}_{-}^{-}$, the past limit of $\mathscr{I}^{-}$, then the terms at future and past timelike infinity should vanish [65] giving

$$
\delta \mathcal{Q}_{\xi}\left(\mathscr{I}^{-}\right)+\delta \mathcal{Q}_{\xi}\left(i^{0}\right)=\delta \mathcal{Q}_{\xi}\left(\mathscr{I}^{+}\right) .
$$

The contribution from spatial infinity in this equation need not vanish in general. ${ }^{27}$

To derive a global conservation law one needs to show that the various limiting integrals exist, and that the contribution $\delta \mathcal{Q}_{\xi}\left(i^{0}\right)$ vanishes. Then integrating in phase space would

\footnotetext{
${ }^{27}$ For example, suppose that in eq. $(2.12) \phi$ is the Minkowski metric, $\delta \phi$ is the linearized Schwarzschild solution, and $\xi^{a}$ is a vector field which asymptotes to one timelike Killing vector field $\tau_{-}^{a}$ of the Minkowski background at $\mathscr{I}^{-}$and to another $\tau_{+}^{a}$ at $\mathscr{I}^{+}$. Then $\delta \mathcal{Q}_{\xi}\left(i^{0}\right)$ is proportional to $P_{a}\left(\tau_{+}^{a}-\tau_{-}^{a}\right)$, where $P_{a}$ is the ADM 4-momentum.
} 
yield a relation of the form

$$
\mathcal{Q}_{\xi}\left(\mathscr{I}^{-}\right)=\mathcal{Q}_{\xi}\left(\mathscr{I}^{+}\right)
$$

which constrains gravitational scattering [65]. We expect that imposing suitable boundary conditions at $i^{0}$ in the definition of $\mathscr{F}$ should eliminate the term $\delta \mathcal{Q}_{\xi}\left(i^{0}\right)$ (this is closely related to the matching conditions proposed in ref. [7]). In addition these boundary conditions should reduce the global symmetry algebra to a diagonal subalgebra of $\mathrm{BMS}^{-} \oplus \mathrm{BMS}^{+}$, with an appropriate identification of $\mathrm{BMS}^{-}$and $\mathrm{BMS}^{+}$, as argued by Strominger [65]. See refs. [67-71] for more detailed analyses of spatial infinity and of the validity of conservation laws of the form (7.3).

Consider now the generalization of this discussion to include horizons [15, 16]. For the black hole formed from gravitational collapse shown in figure 1, and for a representative $\xi^{a}$ of the global symmetry algebra, following the argument that led to eq. (7.1) we obtain

$$
\delta \mathcal{Q}_{\xi}\left(\mathscr{I}^{-}\right)+\delta \mathcal{Q}_{\xi}\left(i^{-}\right)+\delta \mathcal{Q}_{\xi}\left(i^{0}\right)=\delta \mathcal{Q}_{\xi}\left(\mathscr{I}^{+}\right)+\delta \mathcal{Q}_{\xi}\left(i^{+}\right)+\delta \mathcal{Q}_{\xi}\left(\mathcal{H}^{+}\right) .
$$

Here each term is an integral of the form (2.12) over the corresponding hypersurface or an appropriate limit of such integrals converging to one of the points at infinity. The term $\delta \mathcal{Q}_{\xi}\left(i^{-}\right)$can be eliminated as described above. A priori, the symmetry generator $\xi^{a}$ appearing in this equation can have independent limits at the horizon $\mathcal{H}^{+}$and at null infinity $\mathscr{I}^{+} / \mathscr{I}^{-}$. However, just as for $i^{0}$, we expect that imposing appropriate boundary conditions at future timelike infinity $i^{+}$should eliminate the term $\delta \mathcal{Q}_{\xi}\left(i^{+}\right)$, and impose the appropriate relation between the limits of $\xi^{a}$ at $\mathcal{H}^{+}$and at $\mathscr{I}^{+} / \mathscr{I}^{-}$. Note that this viewpoint differs from that of ref. [15], which used the specific prescription of maintaining global Bondi coordinates to link generators at $\mathscr{I}^{-}$to those at $\mathcal{H}^{+}$. However the specific identification for supertranslations obtained there seems inevitable for the case of spherical symmetry. ${ }^{28}$ For more general generators and situations, the appropriate identification of the generators is an interesting question for future study, and will need to be resolved in order to obtain the general form of the global conservation law.

Finally, assuming such an identification has been derived, our explicit expressions for localized charges can be used to obtain an explicit and nonperturbative form of the resulting global conservation law. Integrating eq. (7.4) in phase space and making use of eq. (2.32), this form is

$$
\mathcal{Q}_{\xi}^{\text {loc }}\left(\mathscr{I}_{-}^{+}\right)-\mathcal{Q}_{\xi}^{\text {loc }}\left(\mathscr{I}_{+}^{+}\right)-\mathcal{Q}_{\xi}^{\text {loc }}\left(\mathcal{H}_{+}^{+}\right)=\mathcal{Q}_{\xi}^{\text {loc }}\left(\mathscr{I}_{+}^{-}\right)-\mathcal{Q}_{\xi}^{\text {loc }}\left(\mathscr{I}_{-}^{-}\right) .
$$

Note that the third term on the left hand side is the operator that creates soft graviton hair on a black hole horizon in the quantum theory, when $\xi^{a}$ is a Killing supertranslation

\footnotetext{
${ }^{28}$ With the following minor adjustment: assuming that a supertranslation on $\mathscr{I}^{-}$corresponds to some specific element of the symmetry algebra on $\mathcal{H}^{+}$, as found in ref. [15], the restriction (4.39) implies that the algebra element is a linear combination of a Killing supertranslation and an affine supertranslation, instead of a pure Killing supertranslation. In the notation of eq. (6.37) near $\mathcal{H}_{+}^{+}$, this linear combination will be of the form

$$
\vec{\chi}=\hat{\beta}\left(1+u_{0} e^{-\kappa_{\tau} \tau}\right) \partial_{\tau},
$$

where $u_{0}=u_{0}\left(\theta^{A}\right)$ is determined by the conditions (4.38) or (4.40) at early times. The affine supertranslation correction term does not contribute to localized charges in stationary regions or to global charges.
} 
associated with the asymptotic Killing field near future timelike infinity, as explained by Hawking, Perry and Strominger [15, 16]. Our result (6.27) for this operator improves on existing treatments $[15,16]$ in that it is nonperturbative and not a variation. ${ }^{29}$

\section{Algebra of symmetry generator charges and central charges}

As is well known, the algebra of the global symmetry generator charges $\mathcal{Q}_{\xi}$ under Dirac brackets need not coincide with the symmetry algebra $\mathfrak{g}$ of the vector fields $\xi^{a}$ under Lie brackets, and can instead be a central extension of that algebra [43, 72-74]. This phenomena already arises in classical mechanics [75]. For example, there is a nontrivial central extension for $2+1$ dimensional gravity with a negative cosmological constant with a certain choice of AdS boundary conditions, as shown by Brown and Henneaux [72]. There is no central extension for BMS generators in $3+1$ dimensional general relativity [76], and we show in this section that the same is true for the symmetry algebra of charges at event horizons in general relativity, assuming certain fall off conditions on the shear near future timelike infinity. Thus, there is no central extension of the algebra for the symmetry algebra of global charges derived in this paper.

\subsection{Algebra of symmetry generator charges in general contexts}

We first review in this subsection the theory of central extensions [43, 72-74] in general contexts, and in the following subsection we will apply it to black hole event horizons.

The first step in the computation of the algebra of global charges $\mathcal{Q}_{\xi}$ is the computation of the Dirac bracket. For the specific case of vacuum general relativity at a future event horizon, a careful derivation of the Dirac bracket including the effects of zero modes has been given by Hawking, Perry and Strominger [15]. Here, for the discussion in a general context, we will assume that a Dirac bracket can be found for which the global charges implement the symmetries in the sense $\mathrm{e}^{30}$

$$
\left\{F[\phi], \mathcal{Q}_{\xi}\right\}=\delta_{\xi} F[\phi],
$$

where the variation $\delta_{\xi}$ is defined by

$$
\delta_{\xi} F[\phi]=F\left[\phi+£_{\xi} \phi\right]-F[\phi] .
$$

Here $F$ is any function on the covariant phase space $\overline{\mathscr{F}}$ (i.e. functional of field configurations $\phi)$, and the right hand side is understood to be linearized in $\xi^{a}$. Combining this with the definition (2.12) yields

$$
\left\{\mathcal{Q}_{\xi}, \mathcal{Q}_{\tilde{\xi}}\right\}=-\Omega_{\Sigma}\left(\phi, £_{\xi} \phi, £_{\tilde{\xi}} \phi\right)
$$

An alternative formal derivation of eq. (8.3) is as follows. We write the presymplectic form (2.9) as $\Omega_{\mathscr{A} \mathscr{B}}$, where the indices $\mathscr{A}, \mathscr{B}, \ldots$ represent tensors on $\overline{\mathscr{F}}$. The definition (2.12) of global charges can be written in this notation as

$$
\nabla_{\mathscr{A}} \mathcal{Q}_{\xi}=\Omega_{\mathscr{A} \mathscr{B}} v_{\xi}^{\mathscr{B}},
$$

\footnotetext{
${ }^{29}$ The explicit form of this operator is given by eq. (6.38) above, since the horizon is asymptotically stationary, assuming the fall-off conditions on the shear of appendix G.

${ }^{30}$ Our sign convention for eq. (8.1) is the opposite of that of ref. [15] and agrees with that of ref. [77].
} 
where $v_{\xi}^{\mathscr{B}}$ is the vector field on the covariant phase space that assigns to each solution $\phi$ the linearized solution $£_{\xi} \phi$. We assume the existence of a Dirac bracket on functions $F, G: \overline{\mathscr{F}} \rightarrow \mathbf{R}$ of the form

$$
\{F, G\}=\Omega^{\mathscr{A} \mathscr{B}} \nabla_{\mathscr{A}} F \nabla_{\mathscr{B}} G
$$

where $\Omega^{\mathscr{A} \mathscr{B}}$ satisfies

$$
\Omega_{\mathscr{A} \mathscr{B}} \Omega^{\mathscr{B} \mathscr{C}} \Omega_{\mathscr{C} \mathscr{D}}=\Omega_{\mathscr{A} \mathscr{D}} .
$$

Now inserting the charge definition (8.4) into the bracket (8.5) and using eq. (8.6) gives $\left\{\mathcal{Q}_{\xi}, \mathcal{Q}_{\tilde{\xi}}\right\}=-\Omega_{\mathscr{A} \mathscr{B}} v_{\xi}^{\mathscr{A}} v_{\tilde{\xi}}^{\mathscr{B}}$, which is equivalent to eq. (8.3).

Next, the relation (8.3) can be rewritten using the formulae (2.10) and (8.2) as

$$
\left\{\mathcal{Q}_{\xi}, \mathcal{Q}_{\tilde{\xi}}\right\}=-\int_{\partial \Sigma}\left[\delta_{\xi} \boldsymbol{Q}_{\tilde{\xi}}(\phi)-i_{\tilde{\xi}} \boldsymbol{\theta}\left(\phi, £_{\xi} \phi\right)\right] .
$$

We now specialize to situations where the presymplectic potential $\Theta$ exists, and where the correction term $i_{\xi} \boldsymbol{\Theta}$ in the definition (2.25) of the localized charge vanishes on $\partial \Sigma$ for all $\xi^{a}$. As discussed in section 2.6 above, we expect this to be generically valid when $\Sigma$ is a Cauchy surface. Taking a variation of eq. (2.32) and combining with eqs. (2.13) and (8.7) gives

$$
\left\{\mathcal{Q}_{\xi}, \mathcal{Q}_{\tilde{\xi}}\right\}=-\int_{\partial \Sigma} \delta_{\xi} \mathcal{Q}_{\tilde{\xi}}^{\text {loc }}(\phi)
$$

Now let $\psi_{\varepsilon}: M \rightarrow M$ be the one parameter family of diffeomorphisms that move points along integral curves of $\xi^{a}$. Since these diffeomorphisms preserve the boundaries and the universal structures on the boundaries, and since by construction $\mathcal{Q}_{\tilde{\xi}}^{\text {loc }}$ is local and covariant in the sense of footnote 11, it follows from the argument in that footnote that ${ }^{31}$

$$
\psi_{\varepsilon *} \mathcal{Q}_{\tilde{\xi}}^{\mathrm{loc}}(\phi)=\mathcal{Q}_{\psi_{\varepsilon *} \tilde{\xi}}^{\text {loc }}\left(\psi_{\varepsilon *} \phi\right) .
$$

Now differentiating with respect to $\varepsilon$ and setting $\varepsilon=0$ gives the identity

$$
£_{\xi} \mathcal{Q}_{\tilde{\xi}}^{\mathrm{loc}}(\phi)=\delta_{\xi} \mathcal{Q}_{\tilde{\xi}}^{\mathrm{loc}}(\phi)+\mathcal{Q}_{£_{\xi} \tilde{\xi}}^{\text {loc }}(\phi) .
$$

Inserting this into eq. (8.8) finally gives

$$
\left\{\mathcal{Q}_{\xi}, \mathcal{Q}_{\tilde{\xi}}\right\}=\mathcal{Q}_{[\xi, \tilde{\xi}]}+K_{\xi, \tilde{\xi}},
$$

where $[\xi, \tilde{\xi}]^{a}=£_{\xi} \tilde{\xi}^{a}$ is the Lie bracket and

$$
K_{\xi, \tilde{\xi}}=-\int_{\partial \Sigma} £_{\xi} \mathcal{Q}_{\tilde{\xi}}^{\mathrm{loc}}
$$

Equation (8.11) shows that when the quantity $K_{\xi, \tilde{\xi}}$ is non vanishing, the algebra of charges will differ from the algebra of vector fields.

A priori the quantity $K_{\xi, \tilde{\xi}}$ could depend on the background solution $\phi$. However, a theorem due to Brown and Henneaux [78] shows that there is no such dependence, and so

\footnotetext{
${ }^{31}$ Note that it is important for this argument that $\mathcal{Q}_{\tilde{\xi}}^{\text {loc }}$ does not depend on arbitrary choices such as a choice of representative of an equivalence class in the universal structure.
} 
the algebra of charges consists at most of a central extension of the algebra of vector fields. A formal version of the argument is as follows [43, 79]:

$$
\nabla_{\mathscr{A}} \mathcal{Q}_{[\xi, \tilde{\xi}]}=\Omega_{\mathscr{A} \mathscr{B}} v_{[\xi, \tilde{\xi}]}^{\mathscr{B}}=-\Omega_{\mathscr{A} \mathscr{B}} £_{v_{\xi}} v_{\tilde{\xi}}^{\mathscr{B}}=-£_{v_{\xi}}\left(\Omega_{\mathscr{A} \mathscr{B}} v_{\tilde{\xi}}^{\mathscr{B}}\right)=-£_{v_{\xi}} \nabla_{\mathscr{A}} \mathcal{Q}_{\tilde{\xi}}
$$

Here we have used the charge definition (8.4), then the fact that the mapping $\xi^{a} \rightarrow-v_{\xi}^{\mathscr{A}}$ is a Lie algebra homomorphism, ${ }^{32}$ then the fact that $\Omega_{\mathscr{A} \mathscr{B}}$ is a closed two form on $\overline{\mathscr{F}}$, and finally the definition (8.4) again. Continuing we obtain

$$
\nabla_{\mathscr{A}} \mathcal{Q}_{[\xi, \tilde{\xi}]}=-\nabla_{\mathscr{A}}\left(v_{\xi}^{\mathscr{B}} \nabla_{\mathscr{B}} \mathcal{Q}_{\tilde{\xi}}\right)=-\nabla_{\mathscr{A}}\left(v_{\xi}^{\mathscr{B}} \Omega_{\mathscr{B} \mathscr{C}} v_{\tilde{\xi}}^{\mathscr{C}}\right)=\nabla_{\mathscr{A}}\left\{\mathcal{Q}_{\xi}, \mathcal{Q}_{\tilde{\xi}}\right\}
$$

where we have used eqs. (8.4), (8.5) and (8.6). It follows from eq. (8.11) that $\nabla_{\mathscr{A}} K_{\xi, \tilde{\xi}}=0$, as claimed.

\subsection{Symmetry algebra of global charges at event horizons}

We now show that the contribution ${ }^{33}$ to the central charges (8.12) from a future event horizon vanishes, assuming certain fall off conditions on the shear near future timelike infinity. This generalizes a result of Guo, Hwang and Wu who show that the central charges vanish on the horizon of a stationary, axisymmetric black hole for a large class of generators [80].

Consider a connected component $\mathcal{S}$ of $\partial \Sigma$ which lies in the event horizon $\mathcal{H}^{+}$. Using Cartan's formula together with eq. (6.29) we find that the contribution from $\mathcal{S}$ to the central charges (8.12) can be written as

$$
-\int_{\mathcal{S}} i_{\xi} d \mathcal{Q}_{\tilde{\xi}}^{\text {loc }}=-\frac{1}{8 \pi} \int_{\mathcal{S}} \chi^{i} \varepsilon_{i j k} \hat{D}_{p}\left[\tilde{\chi}^{m} \mathcal{K}_{m}^{p}-\theta \tilde{\chi}^{p}-\tilde{\beta} \ell^{p}\right] .
$$

Now $\mathcal{S}$ cannot lie in the interior of $\mathcal{H}^{+}$, otherwise $\Sigma$ would not be a Cauchy surface. We consider two different cases:

- The cross section $\mathcal{S}$ coincides with a component of the boundary $\mathcal{H}^{+}$, for example the bifurcation two-sphere $\mathcal{H}_{-}^{+}$in an eternal black hole spacetime. Since $\chi^{i}$ must be tangent to $\mathcal{S}$ in this case, as argued in section 4.5 above, it follows that the quantity (8.15) vanishes.

- The cross section $\mathcal{S}$ represents the future asymptotic boundary $\mathcal{H}_{+}^{+}$of $\mathcal{H}^{+}$. Now as discussed in appendix G, event horizons are asymptotically stationary. Assuming exact stationarity and using the condition (3.31), the quantity (8.15) reduces to

$$
-\frac{1}{8 \pi} \int_{\mathcal{S}} \chi^{i} \varepsilon_{i j k} \hat{D}_{p}\left[\tilde{\chi}^{m} \omega_{m} \ell^{p}-\tilde{\beta} \ell^{p}\right]=-\frac{1}{8 \pi} \int_{\mathcal{S}} \chi^{i} \varepsilon_{i j k} £_{\ell}\left[\tilde{\chi}^{m} \omega_{m}-\tilde{\beta}\right] .
$$

\footnotetext{
${ }^{32}$ This follows from the fact that $v_{\xi}$ maps any functional $F[\phi]$ to $F\left[\phi+£_{\xi} \phi\right]-F[\phi]$ to linear order, so $\left[v_{\xi}, v_{\tilde{\xi}}\right] F[\phi]=F\left[\phi+\left(£_{\tilde{\xi}} £_{\xi}-£_{\xi} £_{\tilde{\xi}}\right) \phi\right]-F[\phi]$.

${ }_{33}$ As discussed in sections 2.4 and 2.5 above, a symmetry $\xi^{a}$ can have different limiting forms on different boundaries $\mathcal{B}_{j}$, and more than one can contribute to the central charge (8.12), depending on the Cauchy surface $\Sigma$.
} 
Here $\omega_{m}$ is the rotation one-form (3.32) and we have used eqs. (3.28), (3.14) and (3.31). The Lie derivative in the integrand on the right hand side of eq. (8.16) vanishes, as shown after eq. (6.35), and so the result vanishes. In this analysis we have set the shear $\sigma_{i j}$ and expansion $\theta$ to zero. Assuming instead the falloff conditions $\sigma_{i j}, \theta \sim v^{-p}$ with $p>1$ of appendix $\mathrm{G}$, where $v$ is affine parameter, one can show by an analysis similar to that of appendix $G$ that the contribution of the shear and expansion to the expression (8.15) vanishes in the limit $v \rightarrow \infty$. Hence the contribution from $\mathcal{H}_{+}^{+}$ to the central charge (8.12) vanishes.

\subsection{Symmetry algebras of localized charges}

One can also consider the algebra of localized charges $\mathcal{Q}_{\xi}^{\text {loc }}(\mathcal{S})$. The Poisson bracket of two such charges $\mathcal{Q}_{\xi}^{\text {loc }}(\mathcal{S})$ and $\mathcal{Q}_{\tilde{\xi}}^{\text {loc }}(\tilde{\mathcal{S}})$ will in general depend on the two-surfaces $\mathcal{S}$ and $\tilde{\mathcal{S}}$, but if one specializes to a stationary region of the null surface $\mathcal{N}$ the bracket becomes independent of the two-surfaces. It will be of the form ${ }^{34}$

$$
\left\{\mathcal{Q}_{\xi}^{\mathrm{loc}}(\phi), \mathcal{Q}_{\tilde{\xi}}^{\mathrm{loc}}(\phi)\right\}=\mathcal{Q}_{[\xi, \tilde{\xi}]}^{\mathrm{loc}}(\phi)+K_{\xi, \tilde{\xi}}^{\mathrm{loc}}(\phi),
$$

where the anomalous term $K_{\xi, \tilde{\xi}}^{\text {loc }}(\phi)$ will in general depend on the background solution $\phi$ [81], in contrast to the situation (8.11) for the global charges. While we do not consider the algebra (8.17) in this paper, we note that a recent paper by Haco, Hawking, Perry and Strominger has computed such an algebra for Kerr black holes, and used it to derive the Bekenstein-Hawking entropy $[82,83]$. The symmetry algebra $\mathfrak{g}$ of vector fields used there is not the same as the algebra (4.11) used in this paper, and may be related to the extended algebra we discuss in appendix $\mathrm{H}$.

\section{Discussion, applications and future directions}

In this final section we recap our main results, discuss some implications and applications, and discuss some open questions and future directions.

\subsection{Recap}

In this paper, we have applied the covariant phase space formalism to general relativity with a null boundary. By an appropriate gauge-fixing at the boundary we defined a field configuration space, and derived the conditions for linearized diffeomorphisms to preserve this configuration space. Factoring out by the degeneracies left us with the infinite dimensional symmetry algebra $\mathfrak{g}=\operatorname{diff}\left(S^{2}\right) \ltimes \mathfrak{s}$, where $\mathfrak{s}$ is the set of supertranslations at $\mathcal{N}$ i.e. vector fields $\chi^{i}=f \ell^{i}$ satisfying $£_{\ell}\left(£_{\ell} f+\kappa f\right)=0$. Supertranslations were therefore found to be symmetries of general relativity at general null boundaries. We then calculated the general form of the global conserved charges, and the localized charges and fluxes associated to $\mathfrak{g}$ by way of the Wald-Zoupas prescription. In particular, we found explicit expressions for the supertranslation localized charges and fluxes. These expressions are

\footnotetext{
${ }^{34}$ Barnich and Troessaert have shown that the anomalous term $K_{\xi, \tilde{\xi}}^{\text {loc }}(\phi)$ vanishes for the case of BMS generators at null infinity in $3+1$ general relativity [81].
} 
unique when we impose the condition that the potential $\Theta$ for the presymplectic current on the null surface vanish when the surface is shear free and expansion free.

\subsection{Black holes: localized conservation laws and horizon memory}

We next discuss the implications and interpretation in the event horizon context of the localized conservation laws that we have derived.

As discussed in section 2.6 above, given any two cross sections $\mathcal{S}$ and $\mathcal{S}^{\prime}$ of the event horizon, we have for each symmetry generator a localized conservation law of the form

$$
\int_{\Delta \mathcal{N}} d \mathcal{Q}_{\xi}^{\text {loc }}=\int_{\mathcal{S}^{\prime}} \mathcal{Q}_{\xi}^{\text {loc }}-\int_{\mathcal{S}^{\prime}} \mathcal{Q}_{\xi}^{\text {loc }}
$$

where $\Delta \mathcal{N}$ is the region of $\mathcal{N}$ between $\mathcal{S}$ and $\mathcal{S}^{\prime}$ and explicit expressions for the charge and flux are given in eqs. (6.27) and (6.29). Now since the event horizon has a boundary (either an initial event $\mathcal{P}$ or a bifurcation two-sphere), some of the symmetry generators $\chi^{i}$ of the algebra discussed in section 4 do not preserve the boundary. As discussed in section 4.5, those generators must be excluded from the global algebra $\mathfrak{g}$ that is relevant for global conservations laws. Nevertheless, the conservation law (9.1) is valid for all generators. This is because the derivation of the law (9.1) is local, and is not invalidated if the vector field violates the required boundary conditions at $\partial \mathcal{N}$ if $\partial \mathcal{N}$ is disjoint from $\Delta \mathcal{N}$.

In order to get some insight into the physical interpretation of the charges in (9.1), we specialize to stationary regions. The three different types of generators are:

- Affine supertranslations. The associated charges vanish identically in stationary regions, as noted in section 6.5 above.

- Superrotations or $\operatorname{diff}\left(S^{2}\right)$ generators. The corresponding charges in stationary regions are given by the first term in eq. (6.38) above. The curl (magnetic parity) piece of $X^{A}$ yields the horizon angular momentum multipoles of Ashtekar [84], while the gradient (electric parity) piece gives additional charges.

- Killing supertranslations. The charge in this case is given by the second term in eq. (6.38).

These charges all vanish for a Schwarzschild black hole, except for the $l=m=0$ component of the Killing supertranslation charge in (6.38). ${ }^{35}$ However, as explained in ref. [15], one can turn on an infinite number of non trivial charges by acting on the metric with symmetry transformations. If we write the charges as $\mathcal{Q}_{\xi}^{\text {loc }}\left(\mathcal{S}, g_{a b}\right)$, including the dependence on the metric $g_{a b}$, then it follows from covariance and the fact that the charges are independent of $\mathcal{S}$ in stationary regions that

$$
\mathcal{Q}_{\xi}^{\text {loc }}\left(\mathcal{S}, g_{a b}+£_{\tilde{\xi}} g_{a b}\right)=\mathcal{Q}_{\xi}^{\text {loc }}\left(\mathcal{S}, g_{a b}\right)-\mathcal{Q}_{£_{\tilde{\xi}} \xi}^{\text {loc }}\left(\mathcal{S}, g_{a b}\right)
$$

to linear order in $\tilde{\xi}^{a}$. Hence one can compute the charges on a transformed background in terms of the charges on the original background by making use of the algebra (4.10)

\footnotetext{
${ }^{35}$ Here we define the splitting of a general generator into supertranslation and superrotation pieces by identifying the coordinates in (6.37) with ingoing Eddington-Finkelstein coordinates.
} 
of symmetry generators. It follows from this algebra that acting on the Schwarzschild metric with a superrotation turns on an infinite number of Killing supertranslation charges, and similarly acting with a Killing supertranslation turns on an infinite number of superrotation charges.

We next turn to a consideration of stationary to stationary transitions, which helps to clarify the nature of the charges and conservation laws just as at future null infinity. Suppose that there are two different stationary regions of the horizon separated by a region which is non-stationary. ${ }^{36}$ Then the stationary regions are associated with two different Killing supertranslation algebras $\mathfrak{t}_{1}$ and $\mathfrak{t}_{2}$. This is analogous to the status of Poincaré subalgebras of the BMS algebra at null infinity. Just as there, one can find a finite supertranslation $\sigma$ for which

$$
\mathfrak{t}_{2}=\sigma \mathfrak{t}_{1} \sigma^{-1}
$$

so that the two subalgebras are related by a supertranslation. Specifically, in the notation of eq. (4.35), if the two subalgebras are given by $\alpha-\beta u_{1}=0$ and $\alpha-\beta u_{2}=0$, where $u_{1}$ and $u_{2}$ are functions just of $\theta^{A}$, then one can take $\sigma$ to be the affine supertranslation $u \rightarrow$ $u+\left(u_{2}-u_{1}\right)$. This supertranslation is presumably is related to an analog of gravitational wave memory on the horizon [16]. The details of how such memory can be defined and measured is an interesting topic for future study. The transition is also associated with net changes in (electric parity) superrotation charges, as at $\mathscr{I}^{+}$.

Finally, our formalism does not furnish an analog of the Bondi mass on black hole horizons, that is, a prescription for computing the mass of the black hole at an arbitrary cross section $\mathcal{S}$ of the horizon. This is so for two reasons. First, it would be necessary to specify a preferred symmetry generator (or preferred four-dimensional subgroup of translations for a 4-momentum) from the algebra in order to obtain such a definition. While there is a preferred generator for each stationary region (the Killing vector), in general horizons are non-stationary, and there is no preferred generator or preferred four-dimensional subgroup of translations. Second, even when given a generator associated with a stationary region, the corresponding charge is proportional to the area of the black hole (as used in derivations of the first law), not the mass. In this sense horizons are not similar to future null infinity.

\subsection{The limit to future null infinity}

The symmetry algebra for a general null surface that we have derived is larger than the BMS algebra which applies to the asymptotic boundary of future null infinity $\mathscr{I}^{+}$. An interesting question is how the symmetries and charges of the two algebras are related, for a family of null surfaces that limit to $\mathscr{I}^{+}$in an asymptotically flat spacetime. One might expect that the localized charges $\mathcal{Q}_{\xi}^{\text {loc }}$ have finite limits for a subalgebra of the symmetry algebra isomorphic to the BMS algebra, and that the limits of those charges coincide with the BMS charges. In fact, this does not occur, and none of the localized charges $\mathcal{Q}_{\xi}^{\text {loc }}$ have

\footnotetext{
${ }^{36}$ Actually it is not possible to have the first region be exactly stationary, since by Raychaudhuri's equation in vacuum the expansion $\theta$ must monotonically decrease to zero in affine parameterization; it can only be approximately stationary.
} 
finite limits. This occurs because of our choice of reference solution, in effect a different choice of reference solution is necessary in order for finite limiting charges to be obtained at $\mathscr{I}^{+}$. Details of this comparison will be discussed elsewhere [85].

\subsection{Generalizations}

While our results are specific to $d=4$ spacetime dimensions, they generalize straightforwardly to all spacetime dimensions $d \geq 4$, with appropriate changes in numerical coefficients. Our analysis does not depend on details of Greens functions or on asymptotic fall off conditions which can be dimension dependent. This is in contrast to the situation at future null infinity, where the generalization of the symmetry group, charges and memory to higher dimensions is much more involved [25, 86-88]. Thus supertranslation and base-space diffeomorphism (superrotation) symmetries are universal symmetries of all null surfaces in vacuum general relativity.

It would also be useful to generalize our analysis to allow for the presence of matter. We expect that the symmetry algebra and expressions for charges will not be modified, but that the flux expressions will acquire corrections involving the stress-energy tensor, as in the BMS context.

Generalizations to other theories of gravity will be more involved. In particular, the symmetry algebra obtained from the Wald-Zoupas procedure can depend on the Lagrangian through the explicit expression for the charge variation in eq. (2.18), and may no longer coincide with the specific intrinsic symmetry algebra of section 4 (although it may still posess an intrinsic characterization).

Our symmetry algebra is analogous to the BMS symmetry algebra at future null infinity. In that context it has been suggested that the BMS algebra can be usefully extended to include additional symmetries, which do correspond to soft theorems and to new types of gravitational wave memory [7, 89-91]. However these generators are not obtained from the Wald-Zoupas construction and their status as symmetries on phase space is still unclear. Perhaps the algebra computed here of symmetries on finite null surfaces could be similarly extended.

Finally, as discussed in section 7, a key open question in the black hole context is the restriction on the global algebra of symmetry generators imposed by boundary conditions near future timelike infinity, that should determine the identification of symmetry generators on the horizon and at future null infinity. This identification is necessary in order to formulate the general form of the global conservation law associated with the global charges on the horizon.

\section{Acknowledgments}

We thank Robert M. Wald for helpful discussions, and an anonymous referee for some useful comments. V.C. is supported in part by the Berkeley Center for Theoretical Physics, by the National Science Foundation (award numbers 1214644, 1316783, and 1521446), by FQXI grant RFP3-1323, and by the US Department of Energy under Contract DE-AC0205CH11231. É.É.F. and K.P. are supported in part by NSF grants PHY-1404105 and PHY-1707800 to Cornell University. 


\section{A Orthonormal basis formalism for null surfaces}

In this appendix we translate the definitions and formalism described in section 3 , and some of the results of section 6 , into the language of components on an orthonormal basis. This specialization is often useful in computations, although it does depend on arbitrary choices. We first describe the specializations that occur when one chooses an auxiliary null vector, and then the specializations associated with a complete orthonormal basis.

\section{A.1 Review of structures associated with a choice of auxiliary null vector}

We choose an auxiliary null vector field $n^{a}$ on $\mathcal{N}$ which satisfies

$$
\begin{aligned}
n_{a} n^{a} & =0, \\
n_{a} \ell^{a} & =-1 .
\end{aligned}
$$

The pullback of the covector field $n_{a}$ yields a covector on $\mathcal{N}$

$$
n_{i}=\Pi_{i}^{a} n_{a}
$$

which from eqs. (3.7) and (A.1b) satisfies $n_{i} \ell^{i}=-1 .{ }^{37}$ We define the projection tensor

$$
\pi_{b}^{a}=\delta_{b}^{a}+n^{a} \ell_{b} .
$$

At a given point $p$ the mapping $v^{a} \rightarrow \pi_{b}^{a} v^{b}$ maps vectors into the space of vectors orthogonal to $\ell_{a}$, i.e., into the tangent space $T_{p}(\mathcal{N})$. We write this mapping from $T_{p}(M)$ to $T_{p}(\mathcal{N})$ as

$$
v^{a} \rightarrow \Upsilon_{a}^{i} v^{a} .
$$

The quantities $\Upsilon_{a}^{i}$ and $\Pi_{i}^{a}$ satisfy

$$
\delta_{j}^{i}=\Upsilon_{a}^{i} \Pi_{j}^{a}, \quad \pi_{b}^{a}=\Pi_{i}^{a} \Upsilon_{b}^{i} .
$$

We can now define spacetime tensors that correspond to the induced metric

$$
q_{a b}=\Upsilon_{a}^{i} \Upsilon_{b}^{j} q_{i j}=g_{a b}+2 \ell_{(a} n_{b)},
$$

and shear tensor

$$
\sigma_{a b}=\Upsilon_{a}^{i} \Upsilon_{b}^{j} \sigma_{i j}
$$

These quantities depend on the choice of auxiliary null vector $n_{a}$. We can also define a derivative operator $D_{i}$ on $\mathcal{N}$ by, for a given vector field $v^{i}$ on $\mathcal{N}$,

$$
D_{i} v^{j}=\Pi_{i}^{a} \Upsilon_{b}^{j} \nabla_{a} v^{b} .
$$

Here, on the right hand side, $v^{a}$ is any choice of vector field on $M$ for which $v^{a}=\Pi_{i}^{a} v^{i}$ when evaluated on $\mathcal{N}$. It can be checked that this prescription yields a well defined derivative operator, which depends on the choice of $n_{a}$.

We define the rotation one-form $\omega_{i}$ by

$$
\omega_{i}=-n_{j} \mathcal{K}_{i}^{j} .
$$

From eq. (3.18) this satisfies

$$
\omega_{i} \ell^{i}=\kappa .
$$

As noted in section 3.4 above the rotation one-form depends on $n_{i}$ except when $K_{i j}=0$.

\footnotetext{
${ }^{37}$ Given a covector $n_{i}$ on $\mathcal{N}$ with $n_{i} \ell^{i}=-1, n_{a}$ is uniquely determined by the conditions (A.1a) and (A.2).
} 


\section{A.2 Geometric fields on an orthonormal basis}

We choose on $\mathcal{N}$ a set of basis vectors

$$
\vec{e}_{\hat{\alpha}}=\left(\vec{e}_{\hat{0}}, \vec{e}_{\hat{1}}, \vec{e}_{\hat{A}}\right)=\left(\vec{\ell}, \vec{n}, \vec{e}_{\hat{A}}\right)
$$

where $\hat{A}=2,3$, and where $\vec{\ell}^{2}=\vec{n}^{2}=\vec{\ell} \cdot \vec{e}_{\hat{A}}=\vec{n} \cdot \vec{e}_{\hat{A}}=0, \vec{\ell} \cdot \vec{n}=-1, \vec{e}_{\hat{A}} \cdot \vec{e}_{\hat{B}}=\delta_{\hat{A} \hat{B}}$ on $\mathcal{N}$. We extend the definition of these vectors off $\mathcal{N}$ but do not require them to be orthonormal off $\mathcal{N}$.

We can decompose the covariant derivative of the normal on this basis as

$$
\begin{aligned}
\nabla_{a} \ell_{b}= & \gamma \ell_{a} \ell_{b}+\eta \ell_{a} n_{b}+\tau_{\hat{A}} \ell_{a} e_{b}^{\hat{A}}+\epsilon n_{a} \ell_{b}+\zeta n_{a} n_{b}+\kappa_{\hat{A}} n_{a} e_{b}^{\hat{A}}+\alpha_{\hat{A}} e_{a}^{\hat{A}} \ell_{b}+\iota_{\hat{A}} e_{a}^{\hat{A}} n_{b} \\
& +\left(\frac{1}{2} \theta \delta_{\hat{A} \hat{B}}+\sigma_{\hat{A} \hat{B}}\right) e_{a}^{\hat{A}} e_{b}^{\hat{B}}
\end{aligned}
$$

where $\sigma_{\hat{A} \hat{B}}$ is traceless. Imposing the orthonormality of the basis on the hypersurface gives $\zeta=\iota_{\hat{A}}=0$, while imposing (3.2) gives $\epsilon=-\kappa, \kappa_{\hat{A}}=0$. The induced metric, second fundamental form, Weingarten map and rotation one-form in terms of these quantities are

$$
\begin{aligned}
q_{i j} & =\delta_{\hat{A} \hat{B}} e_{i}^{\hat{A}} e_{j}^{\hat{B}} \\
K_{i j} & =\left(\frac{1}{2} \theta \delta_{\hat{A} \hat{B}}+\sigma_{\hat{A} \hat{B}}\right) e_{i}^{\hat{A}} e_{j}^{\hat{B}}, \\
\mathcal{K}_{i}^{j} & =-\kappa n_{i} \ell^{j}+\alpha_{\hat{A}} e_{i}^{\hat{A}} \ell^{j}+\left(\frac{1}{2} \theta \delta_{\hat{A} \hat{B}}+\sigma_{\hat{A} \hat{B}}\right) e_{i}^{\hat{A}} e^{\hat{B} j}, \\
\omega_{i} & =-\kappa n_{i}+\alpha_{\hat{A}} e_{i}^{\hat{A}} .
\end{aligned}
$$

\section{A.3 Expressions for charges}

A simple expression for the Noether charge in terms of the orthonormal basis can be found by combining eqs. (6.7), (3.16), (4.11a), (3.7) and (D.2):

$$
Q_{\xi}(\mathcal{S})=\frac{1}{8 \pi} \int_{\mathcal{S}} \varepsilon_{i j}\left[n_{b} \ell^{a} \nabla_{a} \xi^{b}\right]
$$

Here the null vector $n_{a}$ has been chosen so that its pullback $n_{i}$ to $\mathcal{N}$ is normal to the cross section $\mathcal{S}$. A similar calculation starting from the localized charge (6.27) gives

$$
\mathcal{Q}_{\xi}^{\text {loc }}(\mathcal{S})=\frac{1}{8 \pi} \int_{\mathcal{S}} \varepsilon_{i j}\left[n_{b} \ell^{a} \nabla_{a} \xi^{b}-\theta \xi^{a} n_{a}\right]
$$

For $\operatorname{diff}(\mathcal{Z})$ generators we have $\xi^{a} n_{a} \widehat{=} 0$, and this charge can be rewritten as

$$
\mathcal{Q}_{X}^{\text {loc }}(\mathcal{S})=-\frac{1}{8 \pi} \int_{\mathcal{S}} \varepsilon_{i j}\left[\ell^{a} \xi^{b} \nabla_{a} n_{b}\right]
$$




\section{B Gauge fixing in the definition of field configuration space}

In this appendix we show that the field configuration space $\mathscr{F}_{\mathrm{p}}$ that we defined is obtained from the larger space $\mathscr{F}_{0}$ by a gauge fixing. Specifically, given a manifold $M$ with boundary $\mathcal{N}$, a complete boundary structure $\mathfrak{p}$ on $\mathcal{N}$, and a metric $g_{a b}$ on $M$ for which $\mathcal{N}$ is null and for which the boundary structure induced by $g_{a b}$ is complete, we show that one can find a diffeomorphism $\psi: M \rightarrow M$ which takes $\mathcal{N}$ into $\mathcal{N}$ for which $\psi_{*} g_{a b}$ lies in $\mathscr{F}_{\mathfrak{p}}$.

Let $\mathfrak{u}$ be the intrinsic structure induced by $\mathfrak{p}$, and $\mathfrak{u}^{\prime}$ be the intrinsic structure induced by the metric $g_{a b}$. By hypothesis, both $\mathfrak{u}$ and $\mathfrak{u}^{\prime}$ are complete. Hence by the argument given in section 4.1 there exists a diffeomorphism $\varphi: \mathcal{N} \rightarrow \mathcal{N}$ which takes $\mathfrak{u}$ to $\mathfrak{u}^{\prime}$. Now choose a diffeomorphism $\psi: M \rightarrow M$ whose restriction to $\mathcal{N}$ is $\varphi$. By acting with $\psi$ on the metric we can without loss of generality assume that $\mathfrak{u}=\mathfrak{u}^{\prime}$.

Now let $\mathfrak{p}^{\prime}$ be the boundary structure induced by $g_{a b}$, and choose representatives $\left(\ell^{a}, \kappa, \hat{\ell}_{a}\right)$ and $\left(\ell^{\prime a}, \kappa^{\prime}, \hat{\ell}_{a}^{\prime}\right)$ of $\mathfrak{p}$ and $\mathfrak{p}^{\prime}$. Since $\mathfrak{u}=\mathfrak{u}^{\prime}$ we can, by adjusting the choice of representative if necessary, take $\ell^{a}=\ell^{\prime a}$ and $\kappa=\kappa^{\prime}$. The two normal covectors must be related by some rescaling of the form $\hat{\ell}_{a}=e^{\lambda} \hat{\ell}_{a}^{\prime}$ for some smooth function $\lambda$ on $\mathcal{N}$. We thus have

$$
g^{a b} \hat{\ell}_{a} \widehat{=} e^{\lambda} \ell^{b}
$$

and we want to show that there exists a diffeomorphism $\psi$ that preserves $\mathfrak{u}^{\prime}$ so that

$$
\left(\psi_{*} g^{a b}\right) \hat{\ell}_{a} \widehat{=} \ell^{b}
$$

By applying $\psi_{*}^{-1}$ to both sides of eq. (B.2), specializing the diffeomorphism so that the induced diffeomorphism $\varphi$ on $\mathcal{N}$ is the identity, and using (B.1), we find that a sufficient condition for (B.2) is that

$$
\psi_{*}^{-1} \hat{\ell}_{a} \widehat{=} e^{-\lambda} \hat{\ell}_{a}
$$

To find a diffeomorphism $\psi$ satisfying (B.3), we need only specify its action to linear order in deviation off the surface $\mathcal{N}$. We can parameterize points near $\mathcal{N}$ to linear order by specifying a point $\mathcal{P}$ on $\mathcal{N}$ and a vector $v^{a}$ at $\mathcal{P}$. We define $\psi$ to be the mapping that takes

$$
\psi:\left(\mathcal{P}, v^{a}\right) \rightarrow\left(\mathcal{P}, v^{a}+\zeta^{a}(\mathcal{P}) \hat{\ell}_{b} v^{b}\right)
$$

where $\zeta^{a}$ is some vector field defined on $\mathcal{N}$. This mapping is well defined despite the fact that representing points near $\mathcal{N}$ as pairs $\left(\mathcal{P}, v^{a}\right)$ is not unique, since components of $v^{a}$ along the surface are annihilated by the term proportional to $\zeta^{a}$. Now computing the pullback of the mapping (B.4) we find that the condition (B.3) will be satisfied if we choose the vector field $\zeta^{a}$ to satisfy

$$
1+\zeta^{a} \hat{\ell}_{a}=e^{\lambda}
$$




\section{Characterization of trivial diffeomorphisms at a null boundary}

In this appendix we show that the charge variation (2.13) vanishes for all cross sections $\mathcal{S}$ of a null boundary, and for all solutions and variations of solutions, if and only if the symmetry $\xi^{a}$ satisfies $\chi^{i}=0$ and $\gamma\left(\xi^{a}\right)=0$, where $\chi^{i}$ is defined by eq. (5.15) and $\gamma$ by eq. (5.16).

The charge variation is given by eq. (6.19), but with $\beta$ replaced by $(\beta+\gamma) / 2$ from eq. (6.5):

$$
\begin{aligned}
\delta \mathcal{Q}_{\xi}= & \frac{1}{16 \pi} \int_{\mathcal{S}} \varepsilon_{i j k}\left[h \chi^{l} \mathcal{K}_{l}{ }^{k}-h \beta\left(\chi^{i}\right) \ell^{k} / 2-h \gamma\left(\xi^{a}\right) \ell^{k} / 2-\chi^{l} \Gamma_{l} \ell^{k}+\chi^{l} £_{\ell} h_{l}{ }^{k}+2 \chi^{l} h^{m}{ }_{l} \mathcal{K}_{m}{ }^{k}\right. \\
& \left.-2 \chi^{l} h^{k}{ }_{m} \mathcal{K}_{l}{ }^{m}-\chi^{k} £_{\ell} h-\chi^{k} h_{i}{ }^{j} \mathcal{K}_{j}{ }^{i}\right] .
\end{aligned}
$$

This expression vanishes if $\chi^{i}$ and $\gamma$ vanish, from eq. (4.11a). Conversely, we want to show that the vanishing of the expression (C.1) for all solutions and variations of solutions forces $\chi^{i}=\gamma=0$.

Fix a cross section $\mathcal{S}$. We make use of the explicit form of the general solution to the vacuum Einstein equations on a null surface given by Hayward [92]. It follows from this solution that, on shell, we can freely specify $h_{i}{ }^{j}$ on $\mathcal{S}$ subject to the constraint (5.26), $£_{\ell} h_{i}{ }^{j}$ subject to the constraint

$$
\ell^{i} £_{\ell} h_{i}^{j}=0,
$$

and the quantity $\Gamma_{i}$ defined by eq. (5.27) subject to the constraint (5.29). We now choose $h_{i}{ }^{j}=0$ and $£_{\ell} h_{i}{ }^{j}=0$. In this case the charge variation (C.1) reduces to

$$
\delta \mathcal{Q}_{\xi}=\frac{1}{16 \pi} \int_{\mathcal{S}} \varepsilon_{i j} \chi^{l} \Gamma_{l}
$$

Since $\Gamma_{l}$ can be chosen arbitrarily on $\mathcal{S}$ subject to eq. (5.29), this forces $\chi^{i}=f \ell^{i}$ on $\mathcal{S}$ for some function $f$. Returning now to eq. (C.1), choosing $h_{i}^{j}=0$, and making use of the constraint (C.2) gives the charge variation

$$
\delta \mathcal{Q}_{\xi}=\frac{1}{16 \pi} \int_{\mathcal{S}} \varepsilon_{i j} f £_{\ell} h
$$

Since $£_{\ell} h$ can be chosen arbitrarily on $\mathcal{S}$, this forces $f$ to vanish on $\mathcal{S}$. Since $\mathcal{S}$ was chosen arbitrarily, $f$ (and therefore $\chi^{i}$ ) must vanish on all of $\mathcal{N}$, and so $\beta=0$ from eq. (4.19). Now reverting to a general $h_{i}{ }^{j}$ and $£_{\ell} h_{i}{ }^{j}$ in eq. (C.1), we obtain the charge variation

$$
\delta \mathcal{Q}_{\xi}=\frac{1}{32 \pi} \int_{\mathcal{S}} \varepsilon_{i j} h \gamma
$$

Since $h$ can be chosen arbitrarily on $\mathcal{S}$, this forces $\gamma=0$ on $\mathcal{S}$. Finally, since the choice of $\mathcal{S}$ was arbitrary, if follows that $\chi^{i}$ and $\gamma$ vanish on all of $\mathcal{N}$. 


\section{Consistency check of symmetry algebra}

In this appendix we verify that for vector fields $\xi^{a}$ satisfying the conditions (4.11) and (5.18) of the symmetry algebra, the corresponding metric perturbation (5.23) satisfies the boundary conditions (5.22) derived in section 5.3.

Taking the Lie derivative of eq. (5.4a) with respect to $\xi^{a}$ gives

$$
£_{\xi} \hat{\ell}_{a} \widehat{=} £_{\xi} g_{a b} \ell^{b}+g_{a b} £_{\xi} \ell^{b} .
$$

Making use of eqs. (5.15), (5.23), (4.11a), (5.16) and (5.18) gives

$$
h_{a b} \ell^{b} \widehat{=}(\gamma-\beta) \hat{\ell}_{a} \widehat{=} 0,
$$

which establishes the condition (5.22a).

Next for simplicity and without loss of generality we specialize to a representative of the boundary structure with $\kappa=0$. We write the definition (5.16) in the form, using (5.14) and (5.4a),

$$
\xi^{b} \nabla_{b} \ell^{a}+\ell^{b} \nabla^{a} \xi_{b} \widehat{=} \gamma \ell^{a},
$$

and take the Lie derivative with respect to $\ell^{a}$. The right hand side becomes $\left(£_{\ell} \gamma\right) \ell^{a}$, which vanishes by eqs. (5.18) and (4.11b). Writing $v^{a}$ for the expression on the left hand side, the left hand side becomes $\ell^{c} \nabla_{c} v^{a}-v^{c} \nabla_{c} \ell^{a}$, and the second term can be written as $\gamma \ell^{c} \nabla_{c} \ell^{a} \hat{=} \gamma \kappa \ell^{a} \widehat{=} 0$. We thus obtain

$$
0 \widehat{=} \ell^{c} \nabla_{c} \xi^{b} \nabla_{b} \ell^{a}+\ell^{c} \xi^{b} \nabla_{c} \nabla_{b} \ell^{a}+\ell^{c} \ell^{b} \nabla_{c} \nabla^{a} \xi_{b} .
$$

The first term can be written using the definition (4.11a) as $-\beta \ell^{b} \nabla_{b} \ell^{a}+\xi^{c} \nabla_{c} \ell^{b} \nabla_{b} \ell^{a} \widehat{=}$ $-\xi^{c} \ell^{b} \nabla_{c} \nabla_{b} \ell^{a}$, where we have used (3.2) and $\kappa=0$. It follows that

$$
0 \widehat{=}-\ell^{c} \xi^{b} R_{c b d a} \ell^{d}+\ell^{c} \ell^{b} \nabla_{c} \nabla_{a} \xi_{b} \widehat{=} \ell^{c} \ell^{b} \nabla_{a} \nabla_{c} \xi_{b},
$$

from which the condition $(5.22 \mathrm{~b})$ follows.

\section{E Choice of reference solution}

As explained in section 2, the dynamics of a theory fix the symmetry generator charges on phase space only up to an overall "constant of integration". To fix that constant of integration, following Wald and Zoupas [6], we choose a reference solution and demand that the charges vanish on that solution. There are two different cases, complete intrinsic structures, and incomplete intrinsic structures associated with nontrivial boundaries $\partial \mathcal{N}$ of the null hypersurface $\mathcal{N}$, as discussed in section 4.5

In the first case of complete intrinsic structures, we choose a one-parameter family of reference solutions $g_{a b}(\varepsilon)$ and demand the limit $\varepsilon \rightarrow 0$ of the charges evaluated on the reference solution vanish. (We use a one parameter family rather than a single solution since our chosen family of solutions does not have a continuous limit as $\varepsilon \rightarrow 0$.) The reference solution is maximally extended Schwarzschild written in Kruskal coordinates

$$
d s^{2}=-2 e^{2 \mu(s)} d U d V+m^{2} \rho(s)^{2} d \Omega^{2},
$$


where $s=U V / m^{2}, m$ is the mass, and $\mu(s)$ and $\rho(s)$ are functions whose exact forms are unimportant for what follows. We also need to specify how this manifold is to be identified with our given boundary structure $(M, \mathcal{N}, \mathfrak{p})$. We identify $\mathcal{N}$ with the horizon $U=0$, and pick $\mathfrak{p}$ to be determined by the representative $\left(\ell^{a}, \hat{\ell}_{a}, \kappa\right)$ where

$$
\begin{aligned}
\hat{\ell}_{a} & =(d U)_{a}, \\
\ell^{a} & =-e^{-2 \mu(0)}\left(\frac{\partial}{\partial V}\right)^{a}, \\
\kappa & =0 .
\end{aligned}
$$

We identify the parameter $\varepsilon$ with the mass $m$ and will take the $m \rightarrow 0$ limit.

We now show that the charge (6.27) integrated over a fixed cross section $\mathcal{S}$ vanishes for the reference solution, in the limit $m \rightarrow 0$, as claimed in section 6.4. The expansion $\theta$ and Weingarten map $\mathcal{K}_{i}^{j}$ vanish for this solution with the choice (E.2) of normal. The charge therefore reduces to

$$
\mathcal{Q}_{\xi}^{\mathrm{loc}}(\mathcal{S})=-\frac{1}{8 \pi} \int_{\mathcal{S}} \varepsilon_{i j k} \beta\left(\chi^{i}\right) \ell^{i}
$$

The only quantity that depends on the metric in this expression is the volume form $\varepsilon_{i j k}$, which from eq. (E.1) is of the form $\varepsilon_{i j k}=m^{2} \varepsilon_{i j k}^{0}$ where $\varepsilon_{i j k}^{0}$ is independent of $m$. Hence $\mathcal{Q}_{\xi}^{\text {loc }}(\mathcal{S}) \rightarrow 0$ as $m \rightarrow 0$ as required.

Note that this conclusion is unchanged if we replace the reference solution $g_{a b}(m)$ with $\psi_{*} g_{a b}(m)$ for any diffeomorphism $\psi: M \rightarrow M$ which preserves the boundary structure $\mathfrak{p}$. The only effect of this change on the argument is to replace $\varepsilon_{i j k}^{0}$ with $\varphi_{*} \varepsilon_{i j k}^{0}$, where $\varphi$ is the restriction of $\psi$ to $\mathcal{N}$, which does not affect the conclusion. Thus the consistency condition $^{38}$ discussed by Wald and Zoupas [6] is satisfied.

Turn now to the second case of a nontrivial boundary $\partial \mathcal{N}$. If the boundary $\partial \mathcal{N}$ is a twosphere, we take the reference solution to be the Schwarzschild solution (E.1), with the hypersurface $\mathcal{N}$ now being restricted to $U>0$, so that the boundary $\partial \mathcal{N}$ is identified with the bifurcation twosphere of Schwarzschild. Apart from this modifications the analysis and conclusions are unchanged.

The case where the boundary $\partial \mathcal{N}$ is a single point $\{\mathcal{P}\}$ is slightly more complicated. We choose the reference solution to be the Schwarzschild solution (E.1) for $U>U_{0}(m)$, and a spherically symmetric ingoing Vaidya solution at earlier advanced times, so that the origin of the event horizon is mapped onto $\mathcal{P}$. The reference boundary structure is chosen to satisfy eqs. (E.2) in the Schwarzschild region, which determines its definition everywhere. If we choose the function $U_{0}(m)$ to go to zero as $m \rightarrow 0$, then the charge (6.27) integrated over a fixed cross section $\mathcal{S}$ is evaluated entirely in the Schwarzschild region for sufficiently small $m$, and the rest of the argument follows as before. Roughly speaking, we are taking the limit of small black holes formed in the distant past to define the reference solution in this case.

\footnotetext{
${ }^{38}$ The charges need not vanish in the $m \rightarrow 0$ limit for the transformed reference solution $\psi(m)_{*} g_{a b}(m)$ which allows the diffeomorphism $\psi$ to depend on $m$. However, there is no physical argument for imposing this more stringent requirement.
} 
Of course, we could dispense with the reference solutions and simply say that we are picking the constant of integration to enforce the expression (6.27) starting from its variation. The reference solutions clarify the physical interpretation of that assumption.

\section{F Consistency of two expressions for flux of localized charge}

In this appendix we show explicitly that the two expressions (6.28) and (6.29) for the flux of the localized charge coincide, as they must from the general Wald-Zoupas framework reviewed in section 2 .

The expression (6.28) was derived from eq. (6.22). The variation in the second term in (6.22) can be replaced with a Lie derivative with respect to $\xi$, from eq. (5.23), giving from the expression (6.24) a contribution to $\Theta_{i j k}$ of

$$
-\frac{1}{8 \pi} £_{\chi}\left(\theta \varepsilon_{i j k}\right)
$$

Using Cartan's formula $£_{v} \boldsymbol{\omega}=i_{v} d \boldsymbol{\omega}+d\left(i_{v} \boldsymbol{\omega}\right)$ and the definition (3.25) of the divergence operator shows that this contribution matches the second term in eq. (6.29). Hence, using the expression (6.16) for $\theta_{i j k}$, it remains to show that

$$
\ell^{f}\left(\nabla_{f} h-\nabla_{e} h_{f}^{e}\right)=2 \hat{D}_{i}\left(\chi^{j} \mathcal{K}_{j}^{i}-\beta \ell^{i}\right) .
$$

Inserting the expression (5.23) for the metric perturbation $h_{a b}$ into the left hand side of eq. (F.2), commuting derivatives and making use of the vacuum equation of motion $R_{a b}=0$ gives the expression

$$
\ell^{f} \nabla_{f}\left(\nabla_{a} \xi^{a}\right)-\ell^{f} \nabla_{e} \nabla^{e} \xi_{f}
$$

It follows from eqs. (5.22) and (3.27) that

$$
\nabla_{a}\left[\left(\nabla^{a} \xi^{b}+\nabla^{b} \xi^{a}\right) \ell_{b}\right]=0,
$$

and simplifying by once again commuting derivatives acting on $\xi^{a}$ and inserting into (F.3) gives that the left hand side of eq. (F.2) is

$$
2 £_{\ell}\left(\nabla_{a} \xi^{a}\right)+\left(\nabla^{a} \xi^{b}+\nabla^{b} \xi^{a}\right) \nabla_{a} \ell_{b} .
$$

Note that this expression is independent of the definition of $\ell^{a}$ off $\mathcal{N}$, by eq. (5.22a).

We now turn to evaluating the right hand side of eq. (F.2). We define the vector

$$
v^{a}=\xi^{a} \nabla_{a} \ell^{b}-\beta \ell^{b},
$$

which satisfies $\hat{\ell}_{a} v^{a}=0$, in terms of which the right hand side can be written as $2 \hat{D}_{i} v^{i}$. We now make use of the relation (3.27) between the three dimensional and four dimensional divergence operators, and the definition (3.26), which yields for the right hand side

$$
2 \nabla_{a} v^{a}+2 n^{b}\left(v^{a} \nabla_{a} \hat{\ell}_{b}+\hat{\ell}_{a} \nabla_{b} v^{a}\right)
$$


Here $n^{b}$ is any null vector field which satisfies $n_{a} \ell^{a}=-1$. Now using the definition (4.11a) of $\beta$ in eq. (F.6) we obtain $v^{a}=\ell^{c} \nabla_{c} \xi^{a}$, and substituting into (F.7) gives

$$
2 \nabla_{a} \ell^{c} \nabla_{c} \xi^{a}+2 \ell^{c} \nabla_{a} \nabla_{c} \xi^{a}+2 n^{b} \ell^{c} \nabla_{a} \hat{\ell}_{b} \nabla_{c} \xi^{a}+2 n^{b} \hat{\ell}_{a} \nabla_{b} \ell^{c} \nabla_{c} \xi^{a}+2 n^{b} \hat{\ell}_{a} \ell^{c} \nabla_{b} \nabla_{c} \xi^{a} .
$$

It remains to show that the expressions (F.5) and (F.8) coincide. Commuting the derivatives in the second term in (F.8) and using the vacuum equation of motion $R_{a b}=0$ shows that this term matches the first term in eq. (F.5). The last term in (F.8) vanishes by eqs. (5.22). In the third term, the derivative acting on $\hat{\ell}_{a}$ is entirely along the surface, since $\ell^{c} \ell_{a} \nabla_{c} \xi^{a}=0$ by eq. (5.22a). Hence we can replace $\hat{\ell}_{a}$ with $\ell_{a}$ in this term, and also in the fourth term. Next, we have that $\ell^{a}$ is hypersurface orthogonal on $\mathcal{N}$, so $\ell_{[a} \nabla_{b} \ell_{c]} \widehat{=} 0$. It follows that $\nabla_{a} \ell_{b} \widehat{=} \nabla_{(a} \ell_{b)}+w_{[a} \ell_{b]}$ for some $w_{a}$ with $w_{a} n^{a}=0$. Substituting this into the first, third and fourth terms in eq. (F.8) we find that the dependence on $w_{a}$ cancels out, so that $\nabla_{a} \ell_{b}$ can be replaced in these terms with $\nabla_{(a} \ell_{b}$. The first term in (F.8) then matches the second term in (F.5). The third and fourth terms can be written as $4 p_{a} \ell_{b} \nabla^{(a} \xi^{b)}$ where $p_{a}=n^{b} \nabla_{(a} \ell_{b}$, which vanishes by eq. (5.22a). Thus the expressions (F.5) and (F.8) coincide as desired.

\section{G Symplectic currents on black holes horizons}

Our explicit expressions for the symplectic current and charges for general null surfaces allow us to establish a number of results about black hole horizons.

First, in vacuum general relativity, the obstruction (2.15) to defining the contribution to a global symmetry generator charge $\mathcal{Q}_{\xi}$ from an integral over a future horizon $\mathcal{H}^{+}$ vanishes,

$$
\int_{\mathcal{H}_{ \pm}^{+}} i_{\xi} \boldsymbol{\omega}=0
$$

as discussed in section 2.4 above, assuming certain fall off conditions on the shear along the horizon at the future boundary $\mathcal{H}_{+}^{+}$, which we now discuss. Consider a cross section $\mathcal{S}$ of the horizon that approaches $\mathcal{H}_{+}^{+}$. The integrand in eq. (G.1) is given explicitly for a null surface in eq. (6.20), and scales as a product of a symmetry generator $\chi^{i}$, times the expansion or shear of the background, times two factors of metric perturbation $h_{i j}$. Denoting an affine parameter along the horizon by $v$, the symmetry generator scales $\sim v$ as $v \rightarrow \infty$, by eq. (4.8). If the shear of the background and perturbations scales as

$$
\sigma_{i j} \sim v^{-p}
$$

for some $p>1$ as $v \rightarrow \infty$, then it follows from eq. (9.2.32) of Wald [50] that the expansion $\theta$ is negligible. Also from eq. (3.15) it follows $h_{i j} \sim v^{-(p-1)}+$ (const), and hence the condition (G.1) will be satisfied at $\mathcal{H}_{+}^{+}$.

Is the condition (G.2) on the late time decay of the shear physically realistic? Consider first linear gravitational perturbations of a Kerr black hole with initial data of compact spatial support. For this case Barack showed that the Weyl scalars $\Psi_{0}$ and $\Psi_{4}$ decay along the horizon at late times like $v^{-7}$ or smaller [93]. It then follows from eqs. (9.2.32) and 
(9.2.33) of Wald [50] that $\sigma_{i j} \sim v^{-6}$. For more general solutions with incoming radiation at $\mathscr{I}^{-}$, we conjecture that imposing that the News tensor fall off along $\mathscr{I}^{-}$as $\sim v^{-p}$ with $p>1$ in the limit $v \rightarrow \infty$ towards $\mathscr{I}_{+}^{-}$will be sufficient to ensure the fall off condition (G.2) along the event horizon, both linearly and nonlinearly. This conjecture is based on the intuition that backscattering should serve to decrease rather than increase the incoming flux at late advanced times $v$.

For eternal black holes with a bifurcation two-sphere $\mathcal{H}_{-}^{+}$, the condition (G.1) will be satisfied at $\mathcal{H}_{-}^{+}$from eqs. (4.40) and (6.20).

Second, we show that the contribution to any global symmetry generator charge $\mathcal{Q}_{\xi}$ from the integral over a future event horizon $\mathcal{H}^{+}$can be expressed in terms of corresponding localized charges $\mathcal{Q}_{\xi}^{\text {loc }}$ evaluated on the components $\mathcal{H}_{ \pm}^{+}$of $\partial \mathcal{H}^{+}$, as discussed in section 2.6 above. This requires the vanishing of the correction term $i_{\xi} \Theta$ in the definition (2.25) of the localized charge:

$$
\int_{\mathcal{H}_{ \pm}^{+}} i_{\xi} \Theta=0
$$

Using the explicit expression (6.26), an argument analogous to that given in the last paragraph shows that the quantity (G.3) vanishes at $\mathcal{H}_{+}^{+}$, under the same assumptions on the shear as above. For eternal black holes with a bifurcation two-sphere $\mathcal{H}_{-}^{+}$, the corresponding integral (G.3) vanishes by the condition (4.40).

\section{H Alternative definition of field configuration space and associated symmetry algebra}

In the body of this paper we have presented a specific definition of a field configuration space $\mathscr{F}$ for general relativity in the presence of a null boundary, and derived from that definition a symmetry algebra and various types of charges. A natural question is whether there is any freedom in the choice of definition of $\mathscr{F}$. In this appendix, we explore a modification of the definition of $\mathscr{F}$, in which we allow a larger set of metrics. A key motivation for this exploration is the fact that new metric variations which are now allowed do not correspond to degeneracies of the symplectic form, and so can be regarded as physical degrees of freedom. We will show that our analysis of the symmetry algebra can be straightforwardly generalized, but that it is not possible to implement the Wald-Zoupas prescription described in section 2.6 to compute localized charges in this context. One can obtain expressions for localized charges but they are not unique.

The starting point for the modified field configuration space definition is to omit the non-affinity $\kappa$ in the definition (4.2) of intrinsic structure $\mathfrak{u}$. Thus, $\mathfrak{u}$ consists of an equivalence class of normals $\ell^{i}$ that are related by rescalings of the form (3.1). The symmetry group is modified by replacing the transformation (4.7a) with an arbitrary smooth mapping $\bar{u}=\bar{u}\left(u, \theta^{A}\right)$, and the algebra (4.11) is modified by dropping the requirement (4.11b). The definition of the boundary structure $\mathfrak{p}$ in section 5 is correspondingly modified by omitting the non-affinity $\kappa$ from the definition (5.3), and omitting the requirement (5.2b) from the definition of the equivalence relation. The definition of the field configuration space $\mathscr{F}_{p}$ is modified by omitting the requirement (5.4b). The conclusions (5.18), (5.19) and (5.20) 
then continue to hold. In particular, a key point is that the arguments of appendix C continue to apply, and so none of the new symmetry generators $\chi^{i}$ on the null surface correspond to degeneracies of the symplectic form.

In the following subsection 5.3, the conditions (5.22b) and (5.29) on variations of the metric are no longer valid. Also the non-affinity $\kappa$ is no longer preserved under variation of the metric, its variation is given by $\delta \kappa=-\Gamma_{a} \ell^{a} / 2$, from eqs. (5.27), (6.10) and (3.18).

The computation of charges in section 6 is modified as follows. The expression (6.7) for the Noether charge is still valid, as is its variation (6.13). In section 6.3, the expression (6.16) for the presymplectic potential $\theta_{i j k}$ is valid, but the subsequent expression (6.17) acquires the extra term $-\varepsilon_{i j k} \Gamma_{a} \ell^{a} /(16 \pi)$, and there is the corresponding correction $\varepsilon_{i j k} \chi^{k} \Gamma_{a} \ell^{a} /(16 \pi)$ to eq. (6.19). In the computation of localized charges, we are unable to find a presymplectic potential $\Theta$ satisfying all the requirements listed in section 2.6. Specifically, if we use the choice (6.24) of the 3 -form $\boldsymbol{\alpha}$, then the extra term in $\boldsymbol{\theta}$ implies that $\Theta$ no longer vanishes on stationary backgrounds. One could cancel this extra term by adding a term proportional to $\kappa \varepsilon_{i j k}$ to $\alpha_{i j k}$, but this term is not invariant under the rescaling (3.1) as it must be. The expression $\kappa-£_{\ell} \ln \theta$ is invariant under rescaling, but from Raychaudhuri's equation in vacuum it is equivalent to $\theta / 2+\sigma_{A B} \sigma^{A B} / \theta$ which is not well defined in the limit $\theta \rightarrow 0$. It does not appear to be possible to find a presymplectic potential $\Theta$ satisfying all the requirements.

Of course, one can drop the requirements related to stationarity, and choose the same expression (6.24) for the 3-form $\boldsymbol{\alpha}$ as before. Then the argument of appendix E shows that, assuming the localized charges $\mathcal{Q}_{\xi}^{\text {loc }}$ vanish on the reference solution, the expressions (6.23) and (6.27) for the localized charge are still valid. However, since we are no longer imposing any assumptions related to stationarity, the relation (2.29) between the flux $d \mathcal{Q}_{\xi}^{\text {loc }}$ and presymplectic potential $\Theta$ need not hold, and the flux will not vanish on stationary backgrounds. In addition, one could have picked other expressions for $\boldsymbol{\alpha}$, so the expression for the localized charge is not unique. It may be possible in this context to find some other criterion that could be used to determine a unique charge expression.

Open Access. This article is distributed under the terms of the Creative Commons Attribution License (CC-BY 4.0), which permits any use, distribution and reproduction in any medium, provided the original author(s) and source are credited.

\section{References}

[1] H. Bondi, M.G.J. van der Burg and A.W.K. Metzner, Gravitational waves in general relativity. 7. Waves from axisymmetric isolated systems, Proc. Roy. Soc. Lond. A269 (1962) 21 [INSPIRE].

[2] R.K. Sachs, Gravitational waves in general relativity. 8. Waves in asymptotically flat space-times, Proc. Roy. Soc. Lond. A270 (1962) 103 [INSPIRE].

[3] R. Sachs, Asymptotic symmetries in gravitational theory, Phys. Rev. 128 (1962) 2851 [INSPIRE].

[4] T. He, P. Mitra, A.P. Porfyriadis and A. Strominger, New Symmetries of Massless QED, JHEP 10 (2014) 112 [arXiv:1407.3789] [inSPIRE]. 
[5] D. Kapec, M. Pate and A. Strominger, New Symmetries of QED, Adv. Theor. Math. Phys. 21 (2017) 1769 [arXiv:1506.02906] [InSPIRE].

[6] R.M. Wald and A. Zoupas, A General definition of 'conserved quantities' in general relativity and other theories of gravity, Phys. Rev. D 61 (2000) 084027 [gr-qc/9911095] [INSPIRE].

[7] A. Strominger, Lectures on the Infrared Structure of Gravity and Gauge Theory, arXiv: 1703.05448 [INSPIRE].

[8] A. Ashtekar and M. Streubel, Symplectic Geometry of Radiative Modes and Conserved Quantities at Null Infinity, Proc. Roy. Soc. Lond. A376 (1981) 585 [INSPIRE].

[9] T. Dray and M. Streubel, Angular momentum at null infinity, Class. Quant. Grav. 1 (1984) 15 [INSPIRE].

[10] L. Donnay, G. Giribet, H.A. González and M. Pino, Extended Symmetries at the Black Hole Horizon, JHEP 09 (2016) 100 [arXiv: 1607.05703] [INSPIRE].

[11] L. Donnay, G. Giribet, H.A. Gonzalez and M. Pino, Supertranslations and Superrotations at the Black Hole Horizon, Phys. Rev. Lett. 116 (2016) 091101 [arXiv: 1511. 08687] [InSPIRE].

[12] C. Eling and Y. Oz, On the Membrane Paradigm and Spontaneous Breaking of Horizon BMS Symmetries, JHEP 07 (2016) 065 [arXiv: 1605.00183] [INSPIRE].

[13] R.-G. Cai, S.-M. Ruan and Y.-L. Zhang, Horizon supertranslation and degenerate black hole solutions, JHEP 09 (2016) 163 [arXiv: 1609.01056] [INSPIRE].

[14] S.W. Hawking, The Information Paradox for Black Holes, 2015, arXiv:1509.01147 [INSPIRE].

[15] S.W. Hawking, M.J. Perry and A. Strominger, Superrotation Charge and Supertranslation Hair on Black Holes, JHEP 05 (2017) 161 [arXiv:1611.09175] [INSPIRE].

[16] S.W. Hawking, M.J. Perry and A. Strominger, Soft Hair on Black Holes, Phys. Rev. Lett. 116 (2016) 231301 [arXiv:1601.00921] [INSPIRE].

[17] S. Carlip, Black Hole Entropy from Bondi-Metzner-Sachs Symmetry at the Horizon, Phys. Rev. Lett. 120 (2018) 101301 [arXiv:1702.04439] [INSPIRE].

[18] M. Blau and M. O'Loughlin, Horizon Shells and BMS-like Soldering Transformations, JHEP 03 (2016) 029 [arXiv: 1512.02858] [INSPIRE].

[19] R.F. Penna, Near-horizon BMS symmetries as fluid symmetries, JHEP 10 (2017) 049 [arXiv: 1703.07382] [INSPIRE].

[20] D. Grumiller and M.M. Sheikh-Jabbari, Membrane Paradigm from Near Horizon Soft Hair, arXiv:1805.11099 [INSPIRE].

[21] J.-i. Koga, Asymptotic symmetries on Killing horizons, Phys. Rev. D 64 (2001) 124012 [gr-qc/0107096] [INSPIRE].

[22] P. Mao, X. Wu and H. Zhang, Soft hairs on isolated horizon implanted by electromagnetic fields, Class. Quant. Grav. 34 (2017) 055003 [arXiv: 1606.03226] [INSPIRE].

[23] R.F. Penna, BMS invariance and the membrane paradigm, JHEP 03 (2016) 023 [arXiv: 1508.06577] [INSPIRE].

[24] A. Strominger and A. Zhiboedov, Gravitational Memory, BMS Supertranslations and Soft Theorems, JHEP 01 (2016) 086 [arXiv:1411.5745] [INSPIRE]. 
[25] S. Hollands, A. Ishibashi and R.M. Wald, BMS Supertranslations and Memory in Four and Higher Dimensions, Class. Quant. Grav. 34 (2017) 155005 [arXiv:1612.03290] [INSPIRE].

[26] R. Bousso, A Covariant entropy conjecture, JHEP 07 (1999) 004 [hep-th/9905177] [INSPIRE].

[27] A.C. Wall, A proof of the generalized second law for rapidly changing fields and arbitrary horizon slices, Phys. Rev. D 85 (2012) 104049 [Erratum ibid. D 87 (2013) 069904] [arXiv: 1105.3445] [INSPIRE].

[28] H. Casini, E. Teste and G. Torroba, Modular Hamiltonians on the null plane and the Markov property of the vacuum state, J. Phys. A 50 (2017) 364001 [arXiv:1703.10656] [InSPIRE].

[29] A.R. Brown, D.A. Roberts, L. Susskind, B. Swingle and Y. Zhao, Complexity, action and black holes, Phys. Rev. D 93 (2016) 086006 [arXiv:1512.04993] [InSPIRE].

[30] L. Lehner, R.C. Myers, E. Poisson and R.D. Sorkin, Gravitational action with null boundaries, Phys. Rev. D 94 (2016) 084046 [arXiv:1609.00207] [InSPIRE].

[31] F. Hopfmüller and L. Freidel, Gravity Degrees of Freedom on a Null Surface, Phys. Rev. D 95 (2017) 104006 [arXiv:1611.03096] [INSPIRE].

[32] W. Wieland, New boundary variables for classical and quantum gravity on a null surface, Class. Quant. Grav. 34 (2017) 215008 [arXiv:1704.07391] [INSPIRE].

[33] W. Wieland, Fock representation of gravitational boundary modes and the discreteness of the area spectrum, Annales Henri Poincaré 18 (2017) 3695 [arXiv:1706.00479] [INSPIRE].

[34] W. Donnelly and L. Freidel, Local subsystems in gauge theory and gravity, JHEP 09 (2016) 102 [arXiv: 1601.04744 ] [INSPIRE].

[35] A.J. Speranza, Local phase space and edge modes for diffeomorphism-invariant theories, JHEP 02 (2018) 021 [arXiv: 1706.05061] [INSPIRE].

[36] F. Hopfmüller and L. Freidel, Null Conservation Laws for Gravity, Phys. Rev. D 97 (2018) 124029 [arXiv: 1802.06135] [INSPIRE].

[37] P.R. Brady, S. Droz, W. Israel and S.M. Morsink, Covariant double null dynamics: (2+2) splitting of the Einstein equations, Class. Quant. Grav. 13 (1996) 2211 [gr-qc/9510040] [INSPIRE].

[38] R.J. Epp, The Symplectic structure of general relativity in the double null (2+2) formalism, gr-qc/9511060 [INSPIRE].

[39] M.P. Reisenberger, The Symplectic 2-form and Poisson bracket of null canonical gravity, gr-qc/0703134 [INSPIRE].

[40] K. Parattu, S. Chakraborty, B.R. Majhi and T. Padmanabhan, A Boundary Term for the Gravitational Action with Null Boundaries, Gen. Rel. Grav. 48 (2016) 94 [arXiv: 1501.01053] [INSPIRE].

[41] C. Crnkovic and E. Witten, Covariant description of canonical formalism in geometrical theories, in Three hundred years of gravitation, S.W. Hawking and W. Israel eds., pp. 676-684. Cambridge University Press (1987) [INSPIRE].

[42] A. Ashtekar, L. Bombelli and O. Reula, The covariant phase space of asymptotically flat gravitational fields, in Mechanics, Analysis and Geometry: 200 Years After Lagrange, M. Francaviglia, ed., North-Holland Delta Series, pp. 417-450, Elsevier, Amsterdam (1991) [INSPIRE]. 
[43] J. Lee and R.M. Wald, Local symmetries and constraints, J. Math. Phys. 31 (1990) 725 [INSPIRE].

[44] R.M. Wald, Black hole entropy is the Noether charge, Phys. Rev. D 48 (1993) R3427 [gr-qc/9307038] [INSPIRE].

[45] I. Khavkine, Covariant phase space, constraints, gauge and the Peierls formula, Int. J. Mod. Phys. A 29 (2014) 1430009 [arXiv:1402.1282] [inSPIRE].

[46] M. Geiller, Edge modes and corner ambiguities in 3d Chern-Simons theory and gravity, Nucl. Phys. B 924 (2017) 312 [arXiv:1703.04748] [INSPIRE].

[47] K. Prabhu, The First Law of Black Hole Mechanics for Fields with Internal Gauge Freedom, Class. Quant. Grav. 34 (2017) 035011 [arXiv:1511.00388] [INSPIRE].

[48] V. Iyer and R.M. Wald, A Comparison of Noether charge and Euclidean methods for computing the entropy of stationary black holes, Phys. Rev. D 52 (1995) 4430 [gr-qc/9503052] [INSPIRE].

[49] M.D. Seifert and R.M. Wald, A General variational principle for spherically symmetric perturbations in diffeomorphism covariant theories, Phys. Rev. D 75 (2007) 084029 [gr-qc/0612121] [INSPIRE].

[50] R.M. Wald, General Relativity, The University of Chicago Press (1984) [InSPIRE].

[51] V. Iyer and R.M. Wald, Some properties of Noether charge and a proposal for dynamical black hole entropy, Phys. Rev. D 50 (1994) 846 [gr-qc/9403028] [InSPIRE].

[52] D. Christodoulou and S. Klainerman, The global nonlinear stability of the Minkowski space, Princeton University Press (1993) [INSPIRE].

[53] L.B. Szabados, Quasi-Local Energy-Momentum and Angular Momentum in General Relativity, Living Rev. Rel. 12 (2009) 4 [INSPIRE].

[54] É.É. Flanagan and D.A. Nichols, Conserved charges of the extended Bondi-Metzner-Sachs algebra, Phys. Rev. D 95 (2017) 044002 [arXiv:1510.03386] [InSPIRE].

[55] T. Jacobson, G. Kang and R.C. Myers, On black hole entropy, Phys. Rev. D 49 (1994) 6587 [gr-qc/9312023] [INSPIRE].

[56] R.M. Wald, On identically closed forms locally constructed from a field, J. Math. Phys. 31 (1990) 2378.

[57] A. Ashtekar, C. Beetle and J. Lewandowski, Geometry of generic isolated horizons, Class. Quant. Grav. 19 (2002) 1195 [gr-qc/0111067] [INSPIRE].

[58] E. Gourgoulhon and J.L. Jaramillo, A 3+1 perspective on null hypersurfaces and isolated horizons, Phys. Rept. 423 (2006) 159 [gr-qc/0503113] [INSPIRE].

[59] J.M. Bardeen, B. Carter and S.W. Hawking, The Four laws of black hole mechanics, Commun. Math. Phys. 31 (1973) 161 [INSPIRE].

[60] A. Ashtekar, Geometry and Physics of Null Infinity, arXiv:1409.1800 [INSPIRE].

[61] M. Hotta, K. Sasaki and T. Sasaki, Diffeomorphism on horizon as an asymptotic isometry of Schwarzschild black hole, Class. Quant. Grav. 18 (2001) 1823 [gr-qc/0011043] [INSPIRE].

[62] D. Lüst, Supertranslations and Holography near the Horizon of Schwarzschild Black Holes, Fortsch. Phys. 66 (2018) 1800001 [arXiv:1711.04582] [INSPIRE]. 
[63] S. Hou, Asymptotic Symmetries of the Null Infinity and the Isolated Horizon, arXiv:1704.05701 [INSPIRE].

[64] E. Morales, On a Second Law of Black Hole Mechanics in a Higher Derivative Theory of Gravity, Ph.D. Thesis, University of Gottingen, (2008).

[65] A. Strominger, On BMS Invariance of Gravitational Scattering, JHEP 07 (2014) 152 [arXiv: 1312.2229] [INSPIRE].

[66] A. Strominger, Black Hole Information Revisited, arXiv:1706.07143 [INSPIRE].

[67] A. Ashtekar and A. Magnon-Ashtekar, Energy-Momentum in General Relativity, Phys. Rev. Lett. 43 (1979) 181 [INSPIRE].

[68] M. Campiglia, Null to time-like infinity Green's functions for asymptotic symmetries in Minkowski spacetime, JHEP 11 (2015) 160 [arXiv:1509.01408] [INSPIRE].

[69] M. Campiglia and R. Eyheralde, Asymptotic U(1) charges at spatial infinity, JHEP 11 (2017) 168 [arXiv:1703.07884] [INSPIRE].

[70] C. Troessaert, The BMS4 algebra at spatial infinity, Class. Quant. Grav. 35 (2018) 074003 [arXiv:1704.06223] [INSPIRE].

[71] K. Prabhu, Conservation of asymptotic charges from past to future null infinity: Maxwell fields, JHEP 10 (2018) 113 [arXiv: 1808.07863] [INSPIRE].

[72] J.D. Brown and M. Henneaux, Central Charges in the Canonical Realization of Asymptotic Symmetries: An Example from Three-Dimensional Gravity, Commun. Math. Phys. 104 (1986) 207 [INSPIRE].

[73] G. Barnich and F. Brandt, Covariant theory of asymptotic symmetries, conservation laws and central charges, Nucl. Phys. B 633 (2002) 3 [hep-th/0111246] [INSPIRE].

[74] G. Barnich and G. Compere, Surface charge algebra in gauge theories and thermodynamic integrability, J. Math. Phys. 49 (2008) 042901 [arXiv: 0708. 2378] [inSPIRE].

[75] V.I. Arnol'd, Mathematical methods of classical mechanics, Graduate Texts in Mathematicals, Springer, New York, NY (1978).

[76] G. Barnich and C. Troessaert, Supertranslations call for superrotations, PoS (CNCFG2010) 010 (2010) [arXiv:1102.4632] [INSPIRE].

[77] H. Goldstein, C. Poole and J. Safko, Classical mechanics, Addison-Wesley (2002).

[78] J.D. Brown and M. Henneaux, On the Poisson Brackets of Differentiable Generators in Classical Field Theory, J. Math. Phys. 27 (1986) 489 [INSPIRE].

[79] A. Seraj, Conserved charges, surface degrees of freedom and black hole entropy, Ph.D. Thesis, IPM, Tehran (2016) [arXiv: 1603.02442] [INSPIRE].

[80] H.-Y. Guo, C.-G. Huang and X.-n. Wu, Noether charge realization of diffeomorphism algebra, Phys. Rev. D 67 (2003) 024031 [gr-qc/0208067] [INSPIRE].

[81] G. Barnich and C. Troessaert, BMS charge algebra, JHEP 12 (2011) 105 [arXiv:1106. 0213] [INSPIRE].

[82] A. Strominger, Progress and Questions in Soft Physics, presentation at Strings 2018, https://indico.oist.jp/indico/event/5/picture/106.pdf.

[83] S. Haco, S.W. Hawking, M.J. Perry and A. Strominger, Black Hole Entropy and Soft Hair, arXiv: 1810.01847 [INSPIRE]. 
[84] A. Ashtekar, J. Engle, T. Pawlowski and C. Van Den Broeck, Multipole moments of isolated horizons, Class. Quant. Grav. 21 (2004) 2549 [gr-qc/0401114] [INSPIRE].

[85] V. Chandrasekaran, É.É. Flanagan and K. Prabhu, in preparation.

[86] D. Kapec, V. Lysov, S. Pasterski and A. Strominger, Higher-Dimensional Supertranslations and Weinberg's Soft Graviton Theorem, arXiv:1502.07644 [INSPIRE].

[87] M. Pate, A.-M. Raclariu and A. Strominger, Gravitational Memory in Higher Dimensions, JHEP 06 (2018) 138 [arXiv: 1712.01204] [INSPIRE].

[88] S. Hollands and A. Ishibashi, Asymptotic flatness and Bondi energy in higher dimensional gravity, J. Math. Phys. 46 (2005) 022503 [gr-qc/0304054] [INSPIRE].

[89] G. Barnich and C. Troessaert, Symmetries of asymptotically flat 4 dimensional spacetimes at null infinity revisited, Phys. Rev. Lett. 105 (2010) 111103 [arXiv:0909.2617] [INSPIRE].

[90] M. Campiglia and A. Laddha, New symmetries for the Gravitational S-matrix, JHEP 04 (2015) 076 [arXiv: 1502.02318] [INSPIRE].

[91] G. Compère, A. Fiorucci and R. Ruzziconi, Superboost transitions, refraction memory and super-Lorentz charge algebra, arXiv:1810.00377 [INSPIRE].

[92] S.A. Hayward, The general solution to the Einstein equations on a null surface, Class. Quant. Grav. 10 (1993) 773.

[93] L. Barack, Late time decay of scalar, electromagnetic and gravitational perturbations outside rotating black holes, Phys. Rev. D 61 (2000) 024026 [gr-qc/9908005] [INSPIRE]. 